\title{
Natural growth of osteochondromas in Hereditary Multiple Osteochondromas
}

Citation for published version (APA):

Staal, $H_{\text {. }}$ (2016). Natural growth of osteochondromas in Hereditary Multiple Osteochondromas. [Doctoral Thesis, Maastricht University]. https://doi.org/10.26481/dis.20160609hs

Document status and date:

Published: 01/01/2016

DOI:

10.26481/dis.20160609hs

Document Version:

Publisher's PDF, also known as Version of record

\section{Please check the document version of this publication:}

- A submitted manuscript is the version of the article upon submission and before peer-review. There can be important differences between the submitted version and the official published version of record.

People interested in the research are advised to contact the author for the final version of the publication, or visit the DOI to the publisher's website.

- The final author version and the galley proof are versions of the publication after peer review.

- The final published version features the final layout of the paper including the volume, issue and page numbers.

Link to publication

\footnotetext{
General rights rights.

- You may freely distribute the URL identifying the publication in the public portal. please follow below link for the End User Agreement:

www.umlib.nl/taverne-license

Take down policy

If you believe that this document breaches copyright please contact us at:

repository@maastrichtuniversity.nl

providing details and we will investigate your claim.
}

Copyright and moral rights for the publications made accessible in the public portal are retained by the authors and/or other copyright owners and it is a condition of accessing publications that users recognise and abide by the legal requirements associated with these

- Users may download and print one copy of any publication from the public portal for the purpose of private study or research.

- You may not further distribute the material or use it for any profit-making activity or commercial gain

If the publication is distributed under the terms of Article $25 \mathrm{fa}$ of the Dutch Copyright Act, indicated by the "Taverne" license above, 


\section{Natural growth of osteochondromas in Hereditary Multiple Osteochondromas}




\section{Colofon}

Copyright (o 2016 by H.M. Staal. All rights reserved.

Cover illustration: pareisaurier, geslacht Bunostegos; Marc Boulay Cover Layout: John Derwall, Heleen Staal

Thesis Layout: www.dlgraphics.nl

Printed by: Schrijen-Lippertz

ISBN: 978-90-824909-1-6

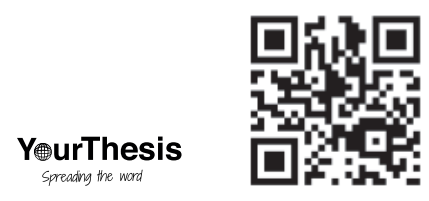

Reproduction of this thesis was supported by the Stichting Kliniek en Wetenschap Orthopaedie, Nederlandse Orthopaedische Vereniging (NOV), Smeets Loopcomfort, Pro-Motion Group BV, Guido Schoenen Orthopedie, Orthopaedie 2000 BV, Livit Orthopedie BV. 


\section{Natural growth of osteochondromas in Hereditary Multiple Osteochondromas}

\section{Proefschrift}

ter verkrijging van de graad van doctor aan de Universiteit Maastricht

op het gezag van de Rector Magnificus, Prof. dr. L.L.G. Soete, volgens het besluit van het College van Decanen in het openbaar te verdedigen op donderdag 9 juni 2016 om 16.00 uur.

door

\section{Heleen Muriel Staal}

Geboren op 19 april 1970 te Maastricht, Nederland 


\section{Promotor}

Prof. dr. Lodewijk W. van Rhijn

\section{Copromotoren}

Dr. T.J.M. Welting,

Dr. M.A. Witlox

\section{Beoordelingscommissie}

Prof. dr. J.H.S. Vles (voorzitter)

Prof. dr. S.K. Bulstra (Universitair Medisch Centrum Groningen)

Prof. dr. M.W. de Haan

Dr. J.B.A. van Mourik (Maxima Medisch Centrum Veldhoven)

Prof. dr. A.M.C.F. Verbunt 




\section{TABLE OF CONTENT}

Chapter one General introduction

Chapter two Current knowledge of osteochondroma formation in $\mathrm{HMO}$; where do osteochondromas originate and in what way is their growth regulated.

Chapter three Distal femoral osteochondromas in patients with $\mathrm{HMO}$, a longitudinal radiological assessment.

Chapter four Skeletal maturity of children with HMO: is diminished stature due to a systemic influence?

Chapter five The microarchitecture of bone in osteochondromas, a pilot study with Micro Computed Tomography Imaging.

Chapter six Dysplasia Epiphysealis Hemimelica:

a histological comparative study with osteochondromas.

Chapter seven The use of whole-body MR imaging in children with $\mathrm{HMO}$, an extended case study in two patients.

Chapter eight Time-lapse technique used to uncover the natural growth of osteochondomas of the wrist in patients with $\mathrm{HMO}$.

Chapter nine General discussion, 139

Future direction and concluding remarks.

Chapter ten Summary 153

Valorization 155

Nederlandse Samenvatting 158

Appendix Curriculum vitae 163

List of publications 165

Dankwoord 


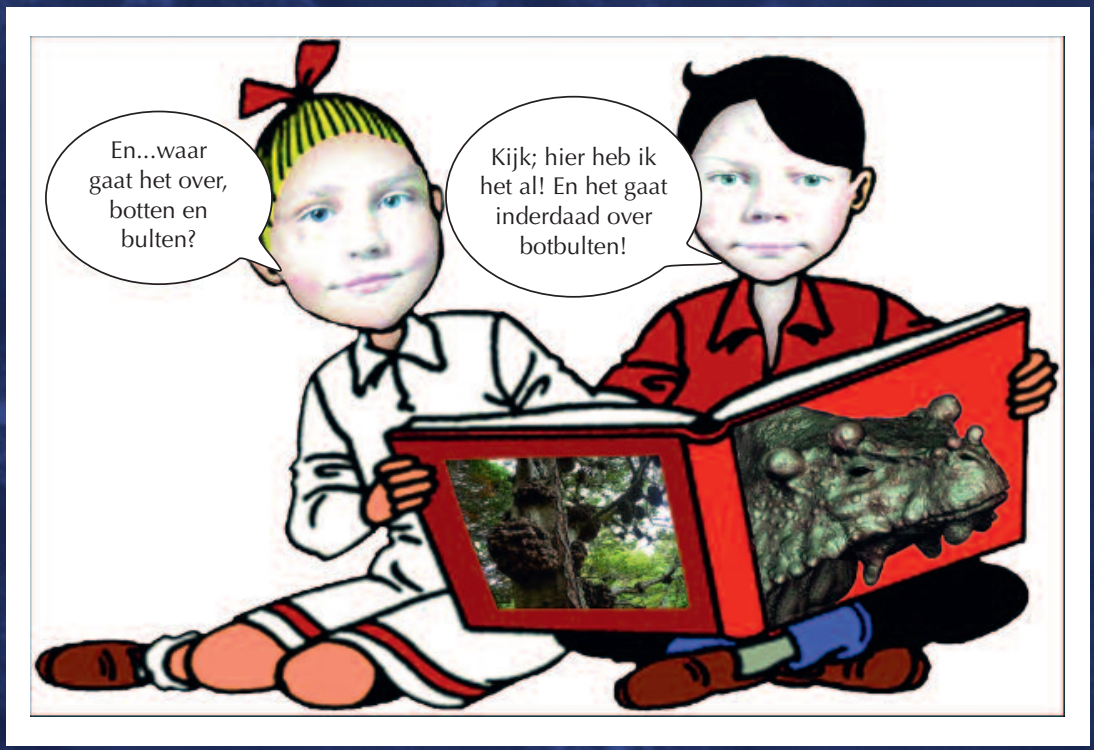




\section{Chapter ONe}

General Introduction 


\section{Chapter 1}

\section{INTRODUCTION}

Normal skeletal growth: the role of the epiphyseal plate.

Epiphyseal plates are found on both sides of long bones. The plates are sub-divided into three well defined zones in which distinct cellular subpopulations are located: the resting zone with progenitor-like immature epiphyseal cells, the proliferation zone with maturating chondrocytes, and the hypertrophic zone with large calcifying and apoptotic chondrocytes (Figure 1). The typical chondrocyte line-up, high cellular proliferation speed and vast amount of matrix deposition, combined with calcification and controlled cell death in the hypertrophic zone causes lengthening of the developing bones. Epiphyseal width decreases at the end of puberty and eventually the epiphyses completely close and are being replaced by bone, leading to growth arrest of the developing individual. The exact mechanism of epiphyseal closure is still not completely understood. However, hormonal influences are evident. Paracrine regulators like parathyroid hormone-related protein (PTHrP) and Indian hedgehog (Ihh) are considered key factors in the regulation of the growth plate $^{1,2}$. The paracrine balance and temporospatial presence of these morphogens is one of the most studied mechanisms of epiphyseal plate biology. Furthermore, sex hormones play an important role. Their levels increase at the onset of puberty, inducing the pubertal growth spurt ${ }^{3-8}$. Oestrogens at high levels promote epiphyseal fusion in females and males ${ }^{9}$. Aforementioned molecular signalling processes are also involved in pathologic skeletal processes leading to skeletal dysplasias such as metaphyseal chondrodysplasias and cleidocranial dysplasia and many others ${ }^{9-11}$. Signifiantly, similar processes are described to be involved in the molecular mechanisms that lead to the formation of osteochondromas indicating that epiphyseal plate biology and osteochondroma formation share common cell biological routes ${ }^{12}$.

\section{Hereditary Multiple Osteochondromas.}

\section{Characteristics}

According to the World Health Organization osteochondromas are defined as a cartilage-capped bony outgrowth on the external surface of long bones consisting of a marrow cavity that is continuous with that of underlying bone ${ }^{13-17}$. 
Histologically, osteochondromas resemble an epiphyseal plate-like build-up with proliferating as well as hypertrophic chondrocytes, often arranged in lined-up clusters. Osteochondromas are usually localized near the metaphysis of bones that develop by endochondral ossification and are commonly found in the distal aspect of the femur, the proximal part of the humerus, and the proximal region of the tibia ${ }^{12-16}$. These sites correlate with the sites of most rapid endochondral ossification. Locations, sizes and number of osteochondromas vary in each patient. In theory every bone element that is formed by endochondral bone formation can be affected. Osteochondromas are not uniquely found in humans, they have been described in many species including cats, dogs, sheep and horses ${ }^{18-22}$. Single osteochondromas are common in the general human population (1 to $2 \%$ ). Hereditary Multiple Osteochondromas ( $\mathrm{HMO}$ ) is the most prevalent genetic skeletal dysplasia. The incidence of $\mathrm{HMO}$ is estimated to be 1 in 50,000 $12-14$. In literature one finds many other names describing this cartilage and bone disorder such as diaphyseal aclasis, osteochondromata, chondral osteoma, osteochondromatosis, multiple cartilaginous exostoses, hereditary multiple exostosis (HME), multiple osteochondroma (MO), deforming chondreodysplasia, osteogenic disease, etc.

Figure 1: Schematic

representation of a femur with a distal osteochondroma and histological images of the epiphyseal cartilage lined up in columns (bottom) and the cartilage on top of the osteochondroma formed in clusters (top).

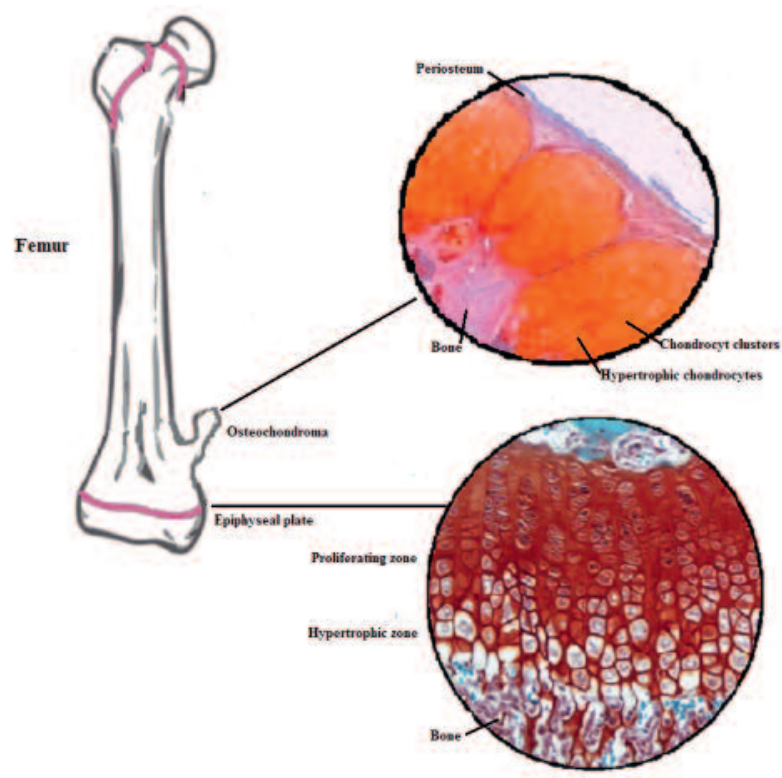




\section{Chapter 1}

\section{Clinical features}

In $\mathrm{HMO}$ the osteochondromas appear shortly after birth and continue to develop throughout childhood and into puberty. By the age of 12, almost all patients have been diagnosed ${ }^{13-15}$. Osteochondromas vary in size, location and shape ${ }^{15-17}$. The growth of osteochondromas can cause clinical problems such as compression on tendons, muscles, nerves, organs and on the spinal cord ${ }^{17,23}$. Figure 2, for example, shows an intra-thoracic osteochondroma compressing the lung. The pressure of the osteochondromas on neighboring tissues and organs can cause pain and dysfunction. Patients can struggle with mobility problems and fatigue ${ }^{23}$. Osteochondromas in the proximity of growth plates seem to cause growth disturbances leading to bone axis deformities (bowing) and luxations. (Figure 3). Osteochondromas themselves or the underlying genetic condition may influence the longitudinal bone growth leading to shortened stature, a common clinical feature observed in $\mathrm{HMO}$ patients ${ }^{24}$.

In general, osteochondromas continue to grow until closure of the growth plates at the end of puberty ${ }^{17,24}$. In adults it is abnormal when an osteochondroma continues to grow. This can be caused by malignant changes in the cartilaginous cap that covers the osteochondroma and can lead to an osteosarcoma. Osteosarcoma is a malignant neoplasm of mesenchymal origin. Malignant transformation of an osteochondroma has been observed in 1 to $5 \%$ of the cases ${ }^{14-17}$. It is advisable to monitor patients even after closure of the growth plates and to remove the osteochondromas that continue to expand after the growth plates have closed because of this possible malignant transformation ${ }^{17}$.

Figure 2: A Ct image of the thoracic cavity showing an osteochondroma (red arrow) of the rib that compresses the underlying lung.

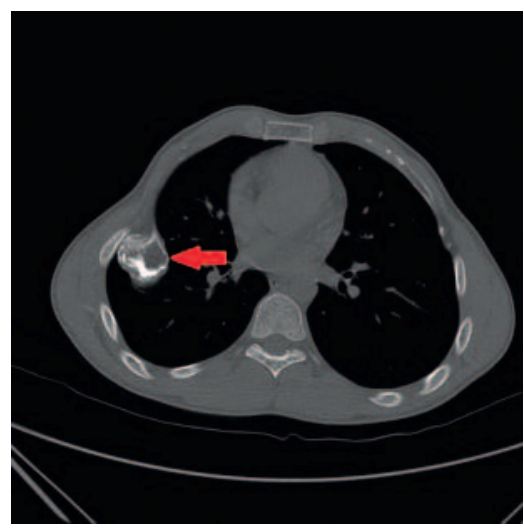



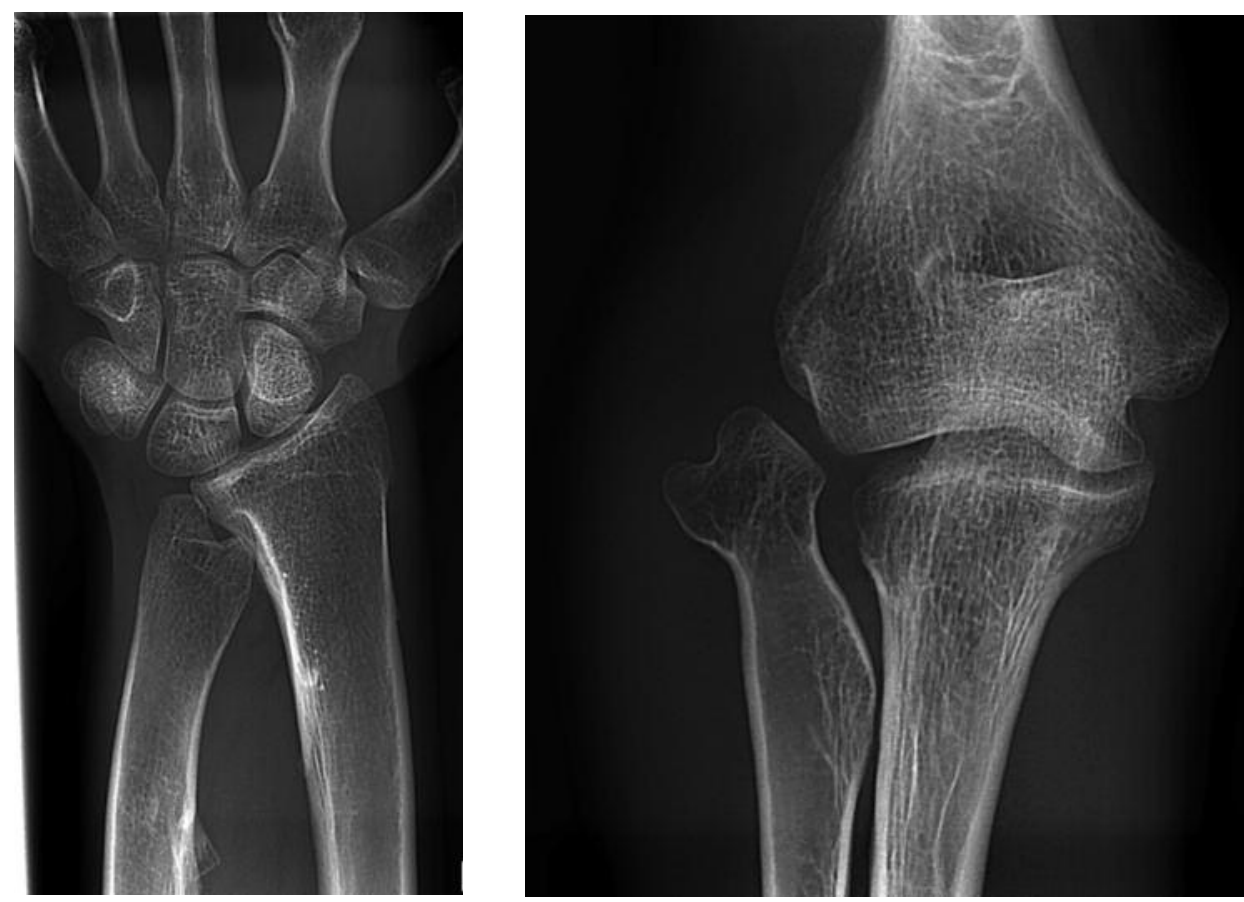

Figure 3: Conventional radiographs of an HMO patient's wrist showing ulnar shorthening and radial deviation (on the left) and radiograph of the ellbow showing radial head dislocation (on the right).

\section{Genetics}

$\mathrm{HMO}$ is an autosomal dominant inherited disease ${ }^{13-17}$. The genetic abnormality leading to $\mathrm{HMO}$ has been determined in the majority of patients. Approximately 60 to $70 \%$ carry a mutation in the exostosin 1 gene (EXT1), 20 to 30\% have an EXT2 gene mutation. The EXT genes encode for glycosyltransferases involved in the biosynthesis of heparan sulfate $\mathrm{e}^{25-27}$. For instance, in laboratory settings genetically modified mouse and zebra fish models have been used. This has led to a better understanding of the role of the EXT genes in the development of the osteochondromas ${ }^{22,25-28}$. In spite of the breakthrough in deciphering the normal functions of exostosin proteins, the cellular and molecular mechanisms leading to the aberrant growth of osteochondromas remain unclear. Genetic testing has given patients options in making choices in reproduction. However, being informed about the genetic background has not altered the orthopedic care. 


\section{Chapter 1}

\section{Treatment}

The only known treatment for osteochondromas up till now is surgical resection of osteochondromas. Surgical corrections can be performed when growth disturbances, like dislocations or axial deformities, appear in $\mathrm{HMO}$ patients. When patients suffer from pain due to growing osteochondromas compressing the soft tissue around them, conservative treatment such as physical therapy and pain management are available. The success rate of the treatment varies from patient to patient ${ }^{23}$. A method to prevent the development of osteochondromas is not available at present.

\section{AIM AND OUTLINE OF THIS THESIS}

The aim of this thesis is to unravel normal development of osteochondromas in order to understand the influence the disease $\mathrm{HMO}$ has on skeletal and general growth. It highlights local and general processes of the disease $\mathrm{HMO}$ with the aim to better understand the origin and development of osteochondromas, to unravel their structure and to improve their visualisation. The thesis starts with an overview of the current knowledge in chapter one.

Chapter two describes the current knowledge and gives a comprehensive literature review on the major pathophysiological theories. It explores the following three questions: What influences the growth of osteochondromas? Do osteochondromas escape Wolff's law and if so, how? What is the place of origin of osteochondromas? The different sections of the chapter evaluate the epidemiology, pathophysiology and histology of osteochondromas. It further focuses on the genetics and the cellular biology. Finally it describes the influence of growth regulatory factors on osteochondromas in $\mathrm{HMO}$.

The natural growth of osteochondromas is further addressed in chapters three and four. The local growth of distal femoral osteochondromas in relation to the growth of the femur itself is addressed in chapter three. Here the development and growth speed of new osteochondromas are compared to the longitudinal growth rate of the host bone. Since most osteochondromas are formed near the epiphysis, 
it was hypothesized that osteochondromas that originate from the epiphyseal periphery continue to grow while the femur is growing. To address this hypothesis we studied the development of osteochondromas in children with $\mathrm{HMO}$ by measuring osteochondromas of the distal femur on long-leg plane radiographs. The lengthening of their femur was compared to the increase in distance between the osteochondroma and the distal end of the femur and the formation of new osteochondromas was recorded. Clinically, short stature is considered as a common feature of $\mathrm{HMO}$, with the majority of affected individuals being below average height. Hormones control skeletal maturation; these hormones might be influenced by the systemic gene defect of the EXT genes. In chapter four we therefore studied the general growth of children with $\mathrm{HMO}$, while hypothesizing that the diminished stature in adults with $\mathrm{HMO}$ is due to a systemic influence leading to early maturation of their epiphyses. Therefore it is expected that the skeletal age in adolescences with $\mathrm{HMO}$ is higher than their corresponding calendar age.

Chapters five and six describe the natural structure of osteochondromas. In chapter five the micro architecture of the bone in osteochondromas is analyzed. Osteochondromas are expected to carry fewer loads than normal bone because of their off-axis position. Therefore the hypothesis is that osteochondromas display a less developed microstructure. To test this hypothesis the bone morphology of osteochondromas was determined using micro-CT scanning and the results were compared to literature values of normal bone. In chapter six the cartilage is analysed. Trevor's disease is a rare condition with asymmetrical limb deformity due to localized overgrowth of cartilage. In literature Trevor's disease is described as an epiphyseal osteochondroma. Therefore the aim of this study was to test the hypothesis that Trevor's disease and HMO are histologically comparable. The study compares the histology of cartilage of children with Trevor's disease with the histology of osteochondromal cartilage of children with HMO.

Chapters seven and eight highlight the visualisation of osteochondromas in children with HMO. Chapter seven describes the diagnostic value of a new development in radiology, the use of whole body MR imaging. Regular conventional radiographs are compared to whole body MR Images of two $\mathrm{HMO}$ affected children. 


\section{Chapter 1}

The images of both methods were evaluated to see if all osteochondromas could be identified and to see if the possible deformity of the long bones was equally visible. The use of this MRI technique could possibly lower the total amount of ionizing radiation that children with $\mathrm{HMO}$ receive because of the frequent radiological follow up. Chapter eight is a digital file that shows the normal growth of wrists of children with $\mathrm{HMO}$ in a time-lapse manner. Regularly each radiograph is assessed as an independent figure, in this single dimension it is difficult to assess actual growth progression. Time-lapse videos give the opportunity to virtually look at the growth of osteochondromas and the wrist articulation development. Conventional radiographic images were therefore combined in a time-lapse technique; the images of the wrist were captured in the same frame, the same contrast and grayscale over time.

This thesis concludes with a general discussion and future directions. It provides a glimpse into future study opportunities, to increase understanding of the disease and to discover a treatment of $\mathrm{HMO}$ at an early stage. 


\section{References}

1. Karimian E, Chagin AS, Sävendahl L, Genetic regulation of the growth plate. Front Endocrinol (Lausanne) 2: 2012, pp113.

2. Emons J, Chagin AS, Sävendahl L, Karperien M, Wit JM, Mechanisms of growth plate maturation and epiphyseal fusion. Horm Res Paediatr 75: 2011, pp 383-391.

3. Juul A, The effects of oestrogens on linear bone growth. Human Reproduction Update, 7 (3), 2001, pp. 303-313

4. Tanner JM, Whitehouse RH, Hughes PC, Carter BS. Relative importance of growth hormone and sex steroids for the growth at puberty of trunk length, limb length, and muscle width in growth hormone-deficient children. The Journal of Pediatrics, 89 (6), 1976, pp. 1000-1008

5. Bundak R, Hindmarsh, PC, Brook CG, Body segments and growth hormone. Archives of Disease in Childhood, 63 (7),1988, pp. 839-840

6. Mortensen HB, Main K, Michaelsen KF, Kastrup KW, Jłrgensen JT, Skakkebaek NE. Predicting and monitoring of growth in children with short stature during the first year of growth hormone treatment. Acta Paediatrica Scandinavica, 80 (12), 1991, pp. 1150-1157

7. Hermanussen M, Gausche R, Keller A, Kiess W, Brabec M, Keller E, Short-term growth response to $\mathrm{GH}$ treatment and considerations upon the limits of short-term growth predictions. Hormone Research, 58 (2), 2002, pp. 71-77

8. Weise M, De-Levi S, Barnes KM, Gafni Rl, Abad V, Baron J, Effects of estrogen on growth plate senescence and epiphyseal fusion. Proceedings of the National Academy of Sciences of the United States of America, 98 (12), 2001, pp. 6871-6876

9. Krakow D, Rimoin DL, The skeletal dysplasias Genetics in Medicine 12, 2010, pp 327-341

10. Baldridge D, Shchelochkov O, Kelley B, Lee B. Signaling pathways in human skeletal dysplasias. Annu Rev Genomics Hum Genet. (11), 2010, pp189-217.

11. Kwok Yeung Tsang K, Wa Tsang S, Chan D, Cheah Yeung Tsang KSE, The chondrocytic journey in endochondral bone growth and skeletal dysplasia, Birth Defects Research Part C: Embryo Today: Reviews, Volume 102 (1), 2014

12. Alman BA. The role of hedgehog signalling in skeletal health and disease. Nat Rev Rheumatol. Sep;11 (9), 2015, pp 552-60.

13. Khurana J, Osteochondroma, World Health Organization Classification of Tumours. Pathology and Genetics of Tumours of Soft Tissue and Bone: 2002, pp 234-236. 


\section{Chapter 1}

14. Bovee JVMG, Osteochondroma. World Health Organization Classification of Tumours. Pathology and Genetics of Tumours of Soft Tissue and Bone. 2002

15. Schmale GA, Conrad EU 3rd, Raskind WH, The natural history of hereditary multiple exostoses. J Bone Joint Surg Am 76: 1994, pp 986-992.

16. Kitsoulis P, Galani V, Stefanaki K, Paraskevas G, Karatzias G, Osteochondromas: review of the clinical, radiological and pathological features. In Vivo 22: 2008, pp 633-646.

17. Bovée JV, Multiple osteochondromas. Orphanet J Rare Dis 3: 32008.

18. Shupe JL, Leone NC, Olson AE, Gardner EJ. Hereditary multiple exostoses: clinicopathologic features of a comparative study in horses and man. American Journal of Veterinary Research, 40(6), 1979, pp 751-757

19. de Brot S, Grau-Roma L, Vidal E, Segalés J. Occurrence of osteochondromatosis (multiple cartilaginous exostoses) in a domestic pig (Sus scrofa domesticus). Journal of Veterinary Diagnostic Investigation September 1, 2013, pp 599-602

20. Pool R, Carrig C, Multiple Cartilaginous Exostoses in a Cat. Veterinary Pathology September vol. 9 no. 5 1972, pp 350-359

21. Wicklund CL, Pauli RM, Johnston D, Hecht JT, Natural history study of hereditary multiple exostoses. Am J Med Genet 55: 1995, pp 43-46.

22. Lind T, Tufaro F, McCormick C, Lindahl U, Lidholt K, The putative tumor suppressors EXT1 and EXT2 are glycosyltransferases required for the biosynthesis of heparan sulfate. J Biol Chem 273: 1998, pp 26265-26268.

23. Goud AL, de Lange J, Scholtes VA, Bulstra SK, Ham SJ. Pain, physical and social functioning, and quality of life in individuals with multiple hereditary exostoses in The Netherlands: a national cohort study. J Bone Joint Surg Am.Jun 6;94(11): 2012, pp 1013-20.

24. Clement ND, Duckworth AD, Baker AD, Porter DE. Skeletal growth patterns in hereditary multiple exostoses: a natural history. J Pediatr Orthop B. 2012 Mar;21(2):150-4.

25. Kitagawa $H$, Shimakawa $H$, Sugahara $K$, The tumor suppressor EXT-like gene EXTL2 encodes an alpha1, 4-N- acetylhexosaminyltransferase that transfers $\mathrm{N}$ acetylgalactosamine and $\mathrm{N}$-acetylglucosamine to the common glycosaminoglycan-protein linkage region. The key enzyme for the chain initiation of heparan sulfate. J Biol Chem 274: 1999, pp13933-13937.

26. McCormick C, Leduc Y, Martindale D, Mattison K, Esford LE, The putative tumour suppressor EXT1 alters the expression of cell-surface heparan sulfate. Nat Genet 19: 1998, pp 158-161. 
27. Wiweger MI, de Andrea CE, Scheepstra KW, Zhao Z, Hogendoorn PC. Possible effects of EXT2 on mesenchymal differentiation—lessons from the zebrafish. Orphanet J Rare Dis. 2014

28. Matsumoto K, Irie F, Mackem S, Yamaguchi Y. A mouse model of chondrocyte-specific somatic mutation reveals a role for Ext1 loss of heterozygosity in multiple hereditary exostoses. Proc Natl Acad Sci USA, Jun 15;107(24), 2010 


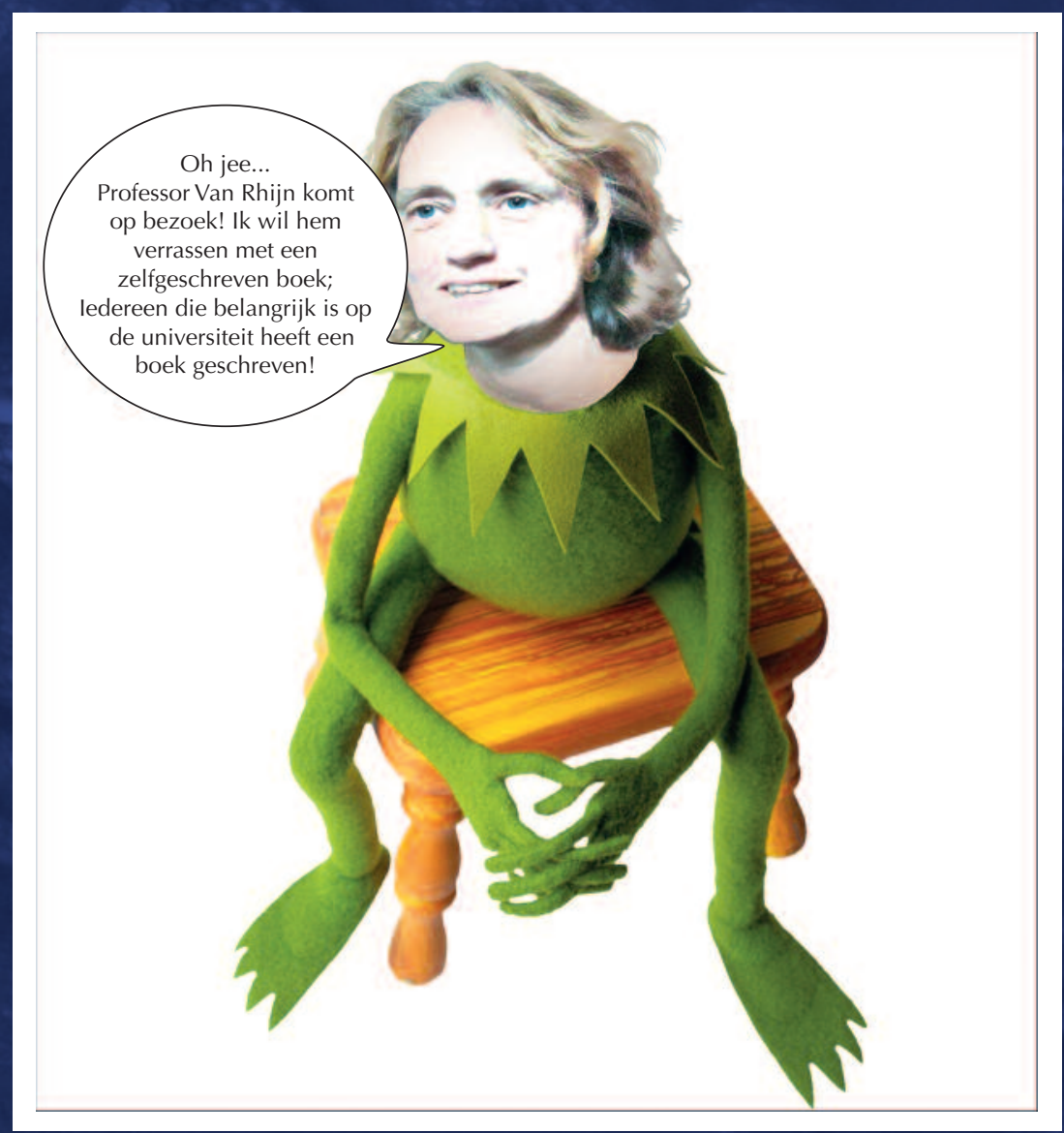




\section{Chapter tWo}

\section{Current knowledge of}

osteochondroma formation in Hereditary Multiple Osteochondromas: where do osteochondromas originate and in what way is their growth regulated?

Heleen M. Staal, M. Adhiambo Witlox, Tristan de Mooij, Pieter J. Emans, S. John Ham, Lodewijk W. van Rhijn, Tim JM. Welting.

Adapted from 


\section{Chapter 2}

\section{Abstract}

Multiple Hereditary Osteochondromas is an autosomal dominant inherited disease causing osteochondromas: growth on the bones of children. The disease is mainly caused by mutated exostosin (EXT)1 or EXT2 genes. These mutations yield nonfunctional EXT-gene products. Lack of functional proteins cause a defect in heparan sulfate synthesis and therefore in proteoglycan modification and cell signalling. It is assumed that a subset of chondrocytes form an osteochondroma, through a growth and differentiation process which is only partially understood. The place of origin of these osteochondroma-forming chondrocytes is still unknown. We also do not know in detail which processes influence the osteochondromas growth, and what shelters the osteochondromas from being resorbed by osteoclast activity. In this paper we systematically review the major pathophysiological theories of osteochondromas, with a focus on the aforementioned knowledge gaps.

Abbreviations: BMP: Bone Morphogenetic Protein; EXT: Exostosin; FGF: Fibroblast Growth Factor; HME: Hereditairy Multiple Exostosis; HMO: Hereditairy Multiple Osteochondromas; HS: Heparan Sulphate; Ihh: Indian hedgehog; LOH: loss of heterozygosity. 


\section{INTRODUCTION}

The World Health Organisation (WHO) defines osteochondromas as a cartilagecapped bony outgrowth on the external surface of long bones. By definition it contains a bone marrow cavity continuing in the normal cavity of the long bone $e^{1,2}$. With a proportion of $30-50 \%$ of all benign bone tumours, it is the most frequently occurring bone lesion. Hereditary multiple osteochondromas (HMO) constitutes a separate, but clinically and radiographically indistinct disease entity that encompasses $10-15 \%$ of all osteochondromas patients. Approximately 1:50,000 people suffer from $\mathrm{HMO}^{3,4}$. The disease is also known as hereditary multiple exostoses, diaphyseal aclasis, osteochondromatosis and multiple cartilaginous exostoses ${ }^{5}$.

The diagnosis of $\mathrm{HMO}$ is based on radiological and clinical presentation of multiple outgrowths (Figure 1), supplemented with, if available, histological evaluation. Approximately $65 \%$ of all patients have a positive family anamnesis ${ }^{6}$. $\mathrm{HMO}$ is an autosomal dominant inherited disease mainly caused by mutated exostosin (EXT)1 or EXT2 genes. This causes a lack of functional proteins influencing heparan-sulphate synthesis, thus affecting the proteoglycan modification and cell signalling, which play a role in osteochondromas growth ${ }^{7}$.

Growth of the osteochondromas occurs as long as a child is growing, and new osteochondromas will form continually. After closure of the growth plates the osteochondromas stop growing and no new ones are formed ${ }^{5}$. Both sessile and pedunculated osteochondromas have been described. Through its shape, the pedunculated more than the sessile variant can compromise overlying tissue and therefore has a greater risk of becoming symptomatic ${ }^{8,9}$. The osteochondromas can lead to compression on tendons, nerves, muscles, ligaments and on the spinal cord. Patients may experience pain or fatigue. Growing osteochondromas are known to cause a set of growth anomalies, including Madelung-like deformity (40-60\%), unequal limb length (10-50\%), joint deformity (2-55\%) and a disproportionally short stature $(37-45 \%)^{3,6,10}$.

Furthermore osteochondromas can fracture (5\%) and they can cause vascular problems, abnormal scar formation, bursa formation, and joint impingement ${ }^{10-13}$. The direction of growth of the osteochondromas is pointed away from the adjacent 


\section{Chapter 2}

growth plate and away from the adjacent joint ${ }^{14}$; they are not in line with the axis of the bone and are therefore not submitted to the axial load. We know from normal bone formation that non-loaded bone will remodel according to Wolff's law. This implies that we expect osteochondromas to be remodeled by creeping substitution and to eventually disappear as a result of osteoclast resorption ${ }^{15,16}$. Until now it is still unknown why osteochondromas after formation do not disappear.

Apart from the unknown mechanism of growth, the place of origin of osteochondromas also remains unclear. Most exostoses are found in the metaphysis under the periosteum, suggesting a metaphyseal origin. However, epiphyseal-like cartilage is found on top, suggesting an epiphyseal origin ${ }^{15}$. There is no medication to cure osteochondromas or to slow their growth. Non-recurrence on site is only ensured after radical surgical removal of the osteochondromas. However, removal of osteochondromas in a skeletally immature patient may lead to epiphyseal damage and growth deformities ${ }^{16,17}$.

The aim of this review is to explore literature in the light of the following clinically raised questions: What factors influence the growth of osteochondromas? Do osteochondromas escape Wolff's law and if so, in what way? What is the place of origin of osteochondromas? In order to answer these questions best as possible, the epidemiology, pathophysiology, marker expression and growth regulation are discussed, and the major pathophysiological theories are reviewed and put into historical perspective.

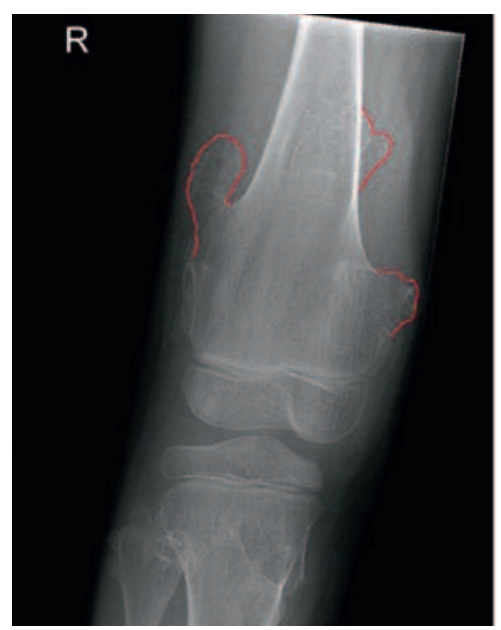

Figure 1: X-ray AP view of the right knee of adolescent female patient with $\mathrm{HMO}$, the lines on the distal femur mark the bony outgrowth of the osteochondromas. 


\section{EPIDEMIOLOGY OF OSTEOCHONDROMAS}

Osteochondromas are a common isolated bony outgrow of the long bones. It affects $1-3 \%$ of the general population. About 10-15\% of these osteochondromas are in the context of the genetic form, Hereditary Multiple Osteochondromas. $\mathrm{HMO}$ has its onset from early infancy to puberty. The osteochondromas ceases to grow and calcifies when the patient reaches skeletal maturity. Thereafter, no new osteochondromas develop ${ }^{4,5}$. The patients in average suffer from 15-18 osteochondromas, but up to a number of 80 osteochondromas have been described. The metaphysis of the tibia, femur and humerus is the most common location ${ }^{18-20}$.

Affliction is usually symmetrical. Caucasians are more often affected than other races, affecting 0.9 - 2 individuals per 100,000. Caucasian men in particular have a higher predilection to suffer from $\mathrm{HMO}^{10,21}$. Male predilection (1.5: 1), however, is possibly due to an easier overlooked milder female phenotype ${ }^{21}$. The osteochondromas have a cartilage cap; the thickness of the cap differs and ranges from 1-2 mm to several centimetres. Increasing thickness correlates with pain and with chondrosarcomatous potential in adults. These osteochondromas often have a more proximal location and a larger size $\mathrm{e}^{1,2,18,22}$. HMO patients have a 1-3\% risk of malignancy and the progression to malignancy is quicker than in non-hereditary (solitary) counterparts ${ }^{1-3,6,10,23,24}$.

\section{Pathophysiology of osteochondromas}

\section{Histology}

Osteochondromas have a strikingly consistent morphology, typically forming a cylinder pointing at various angles away from the epiphyseal disc and the joint. An osteochondroma of a skeletally immature patient consists of a bony stalk and a cartilage cap. The cap is lineated peripherally with the perichondrium, which is continuous with the periosteum of the underlying bone. The cortex of the stalk is in continuity with the cortex of the normal bone, thus creating a continuous medullary cavity. In the skeletally immature patient the medullary cavity is delineated with the cartilaginous cap. The cap has the histological appearance of an epiphyseal growth plate with chondrocytes lined up in columns (Figure 2) ${ }^{18,24}$. 


\section{Chapter 2}

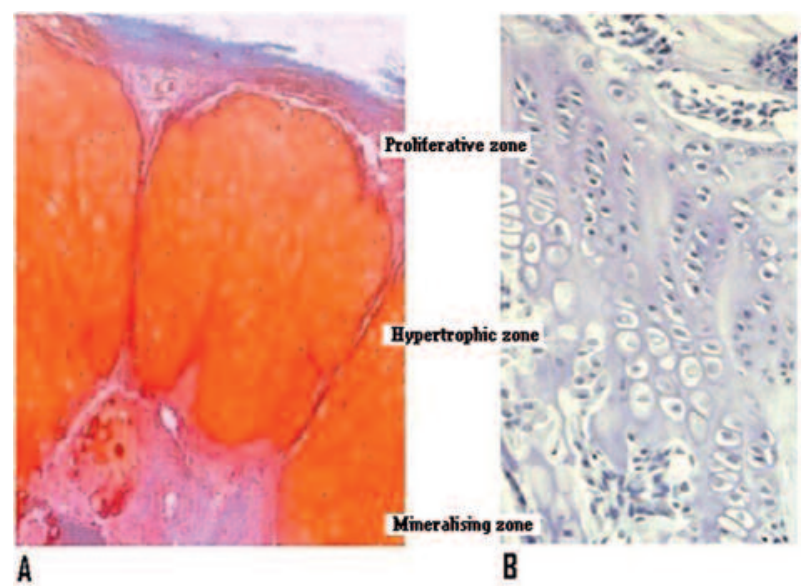

Figure 2: (A) Saffranine-O staining of the cartilaginous cap of an osteochondroma, showing the presence of similar zones as the mouse epiphysis but in separated clusters. (B) Haematoxiline staining of the epiphysis of a 6-week-old mouse as a representative example of a general growth plate.

\section{Genetics}

$\mathrm{HMO}$ is an autosomal dominant inherited condition caused by mutations in the exostosin (EXT) 1 and EXT2 genes. The EXT gene family comprises six members, all located on different chromosomes and chromosomal regions ${ }^{25-27}$. EXT1 and EXT2 mutations together explain over $90 \%$ of all cases of $\mathrm{HMO}^{28,29}$. Mutations in EXT-1 account for $44-70 \%$ of the $\mathrm{HMO}$ cases and in $27-40 \%$ mutations in EXT-2 are causative for HMO. In a subset of patients both genes are affected ${ }^{21,30-32}$. In males, mutations in EXT-1 lead to a more severe HMO phenotype compared to mutations in EXT2. Typically male carriers of mutations in EXT1 display more and bigger osteochondromas ${ }^{33-39}$.

The risk of malignant transformation cannot be linked to these specific genetic mutation. Genetically mutated mice carrying Ext1(+/-) as well as Ext2(+/-) heterozygous mutations mimic the genetic status of human $\mathrm{HMO}$ and have shown to be able to format osteochondromas. The formation of stereotypical osteochondromas seems to require a significant, but not complete, loss of Ext expression ${ }^{40}$. Despite the identification of causative genes, the pathogenesis of $\mathrm{HMO}$ remains unclear. 


\section{Cellular biology}

EXT1 and EXT2 gene products are type II transmembrane proteins. They contain a single 17 amino acid long transmembrane domain and a short amino-terminal cytoplasmatic tail. EXT1 and EXT2 form a complex (EXT1/2) in the Golgi apparatus, acting as glycotransferases ${ }^{41-43}$. Over-expressed EXT1 or EXT2 is accumulated in the endoplasmic reticulum. Only when expressed in synchronous amounts, EXT1/2 complexes are transported to the Golgi apparatus and display maximum catalytic activity $^{43-45}$. They seem to play a role in the heparan sulphate (HS) synthesis.

The synthesis of elongated heparan sulphate (HS) chains involves a polymerisation process that is carried out in different sequential steps. The process starts with a so-called priming step that prepares the subsequent polymerization. Thereafter elongation is initiated by definitive polymerisation of repeating disaccharide units. The EXT proteins are involved in two different steps in this biosynthetic process. EXT-L2 is the glycosyltransferase, which is critically involved in the HS specific polymerisation of a $\mathrm{N}$-acetylglucosamine (GICNAc) residue to a HSspecific tetrasaccharide linker. This initial polymerisation of the GlcNAc residue is a prerequisite for further HS chain elongation catalysed by EXT1 and EXT2 40,43,46-54.

Chain elongation by EXT1/2 involves the alternating polymerization of GICA (glucoronic acid) and GlcNAc dissaccharide units, maturing the functional HS polymer. When the HS polymerisation process is completed, the proteoglycans are transported to the cell surface and located in the extracellular matrix or at the cell surface, where they function in high affinity binding of growth factors, cytokines, extra cellular enzymes and even viral enzymes ${ }^{43,49,55-57 .}$

As only EXT1 and EXT2 are specifically associated with $\mathrm{HMO}$, the major mechanism that is believed to underlie the HMO pathogenesis involves impaired HS-polymerase activity by dysfunctional EXT1/2 activity, leading to improper HS synthesis and aberrant cell signalling due to improper binding of essential signalling molecules. 


\section{Chapter 2}

\section{WHAT INFLUENCES THE GROWTH OF OSTEOCHONDROMAS?}

From clinical follow-up it is known that osteochondromas sprout and grow while the patient is actively growing. After closure of the growth plates the osteochondromas stop growing and no new osteochondromas are formed ${ }^{5,18}$. This raises the question how the growth of osteochondromas is regulated. To answer this question we focus on the influence of mutated EXT genes on the signalling pathways and the major regulatory systems of normal growth regulation.

In patients with $\mathrm{HMO}$ we know that the mutations in either EXT1 or EXT2 result in reduction or absence of $\mathrm{HS}$ in the cartilage compartment of the osteochondroma. This impaired HS synthesis has been linked to disturbed cellular signalling responses leading to growth disturbance of chondrocytes and possibly to the formation of osteochondromas $^{15,40,58,59}$.

There are two major classes of heparan sulphate proteoglycans (HSPGs). Glypicans are located at the cell surface and are glycosylphosphatidylinositol (GPI)linked molecules, solely bearing heparan sulphate. The other main class of signalling HSPGs are syndecans, which are transmembrane proteins decorated with both chondroitin sulphate and heparan sulphate ${ }^{60}$. HS and HSPG act as co-receptors for several growth factors, including bone morphogenic proteins (BMPs), fibroblast growth factors (FGFs), Wingless-members (Wnt), transforming growth factor B(TGFB) and Indian hedgehog (Ihh ${ }^{61-69}$.

Normal endochondral ossification of long bones is a highly regulated process characterized by proliferation of chondrocytes, differentiation, calcification, and programmed cell death. The epiphysis of a long bone is divided into three welldefined zones in which these cellular processes take place: the resting zone with the immature cells (also known as the germinal zone), the proliferating zone with more mature chondrocytes, and the hypertrophic zone with large calcifying and apoptotic chondrocytes. In the growing child the growth plate matures. During this process the hypertrophic cells synthesize collagen type $\mathrm{X}$ and then undergo calcification and cell death. After degradation of calcified cartilage by chondroclasts, the resulting cavities are invaded gradually by osteoblasts secreting bone matrix. At 
the end of puberty, the width of the epiphysis decreases and eventually the epiphysis is completely closed and replaced by bone. This process is controlled by various endocrine, autocrine, and paracrine factors ${ }^{70}$.

The exact mechanism of epiphyseal fusion is still not completely understood. Paracrine regulators like parathyroid hormone-related protein (PTHrP) and Indian hedgehog (Ihh) are considered key factors in the regulation of the growth plate ${ }^{71}$.

These growth factors coordinate endochondral ossification by regulating chondrocyte proliferation. Looking at one of the major regulatory systems we zoom in on the possible effects of the HMO-associated EXT1 and EXT2 gene mutations on the Indian hedgehog - parathyroid hormone related protein feedback loop.

\section{$\mathrm{HMO}$ related to the Indian hedgehog/parathyroid hormone-related protein signalling}

Indian hedgehog (Ihh) seems to orchestrate the chondrocyte proliferation and differentiation and the osteoblast differentiation ${ }^{72}$. Ihh is expressed and secreted by post-mitotic hypertrophic chondrocytes simultaneously with expression of the parathyroid hormone-related protein receptor (PPR) during the bone formation. Ihh diffuses throughout the growth plate and binds to its receptor, Patched-1 (Ptc-1) expressed by chondrocytes in the resting zone. This, in turn, activates downstream signalling that in turn leads to elevation of PTHrP expression ${ }^{73}$. Since inactivation of either Ihh or PPR in chondrocytes leads to abrupt fusion of the epiphyseal growth plate in mice ${ }^{74,75}$, it is suggested that the loop is crucial for maintaining the growth plate in the open phase. In humans inactivating mutation in Ihh results in acrocapitofemoral dysplasia, which is associated with premature closure of the growth plates ${ }^{76}$.

EXT1 and EXT2 are expressed in the proliferating and the hypertrophic zones of the growth plate, and are responsible for an extracellular heparan sulphate proteoglycan (HSPG) gradient. Their expression coincides with the onset of Indian hedgehog signalling. HMO associated mutations in EXT1 and EXT2 may lead to abnormal HS gradient formation in the growth plate ${ }^{66,71}$. De Andrea et al. showed in different studies of human growth plates and of different proteoglycan-deficient zebra fish mutants the disrupted diffusion gradients of morphogens and signal transduction in the epiphyseal growth plate ${ }^{77}$. As diffusion of Ihh is HSPG- 


\section{Chapter 2}

dependent, proliferation zone EXT-/- chondrocytes could "encounter" an abnormal Ihh signal, leading to abnormal proliferation ${ }^{78,79}$. Evidence put forth independently by Hameetman and Benoist-Lasselin shows the presence of Ihh in the cartilage cap of osteochondromas and proves Ihh signalling despite the lack of EXT proteins ${ }^{80-82}$.

The above suggests an influence of HS and Ext in $\mathrm{HMO}$ on the Indian hedgehog/parathyroid hormone-related protein signalling, possibly leading to premature closing of the epiphysis and thereby declaring the short stature in $\mathrm{HMO}$ patients.

In addition to the hedgehog proteins and parathyroid hormone-related peptide, other important local regulators of the epiphysis are the bone morphogenic proteins (BMPs) and the fibroblast growth factors (FGFs). Further local factors such as vascularisation, vitamin $\mathrm{D}$ and transforming growth factor beta (TGF- $\beta$ ) are not discussed in this context because they have no known relation with the EXT genes or HS. Also the systemic factors such as the GH/IGF-I system, glucocorticoids and oestrogens are not discussed.

$\mathrm{HMO}$ related to para/autocrine regulators; the bone morphogenic proteins (BMPs), and the fibroblast growth factors (FGFs).

BMPs have a role in every stage of endochondral bone formation and angiogenesis ${ }^{83,84}$. Lack of BMPs and/or their receptors in early stages have been shown to result in failure in mesenchymal condensation or digit formation in mice ${ }^{85-}$ ${ }^{87}$. In a later stage the BMP proteins are expressed in the perichondrium as well as hypertrophic and proliferative chondrocytes. Indian hedgehog expression in prehypertrophic chondrocytes increases through BMP signaling, thereby increasing both the rate of chondrocyte proliferation and the length of proliferative columns ${ }^{70,88}$. Since HS and HSPG act as co-receptors for BMPs in HMO patient; we therefore expect a decrease in the rate of chondrocyte proliferation and a shortening of the proliferative columns, which may lead to shortage or axial deviation (in case of partially decreased growth rate) of the long bones.

Other local growth factors depending on the HSPGs for cell-signalling activity are the fibroblast growth factors (FGFs). FGFs are essential for normal embryonic development.. FGF receptor 3 (FGFR3) is expressed in the resting zone of the epiphyseal disc, where it promotes hypertrophic differentiation and decreases 
proliferation. In mice it has been shown that FGFs can act as antagonists of BMP signalling and negatively regulate Ihh expression. FGFs acting via FGF receptor-3 (FGFR3) are the key negative regulators in chondrocyte proliferation. Mutations in FGFR3 lead to achondroplasia or hypochondroplasia ${ }^{71}$. The expression of FGFs and their receptors in postnatal growth plate cartilage suggests that these proteins contribute to growth plate senescence and thus help to determine the size of the adult skeleton ${ }^{70,89}$. In relation to HMO, EXT mutation and thus HSPG deficiency would lead to a functional FGFR3 null state. Bovée et al. showed defective (mostly absent) expression of FGF2, FGFR1, FGFR3 in osteochondromas, presumably allowing skeletal overgrowth at the site of the osteochondromas ${ }^{90}$.

In conclusion, in HMO with mutations in either EXT1 or EXT2 it is likely that the absence or reduction of HS disturbs the three major regulatory systems of epiphyseal growth, being the Indian hedgehog/ parathyroid hormone related protein feedback loop, the BMPs and the fibroblast growth factors. One of the striking observations is that the general genetic defect in all cells does not induce osteochondromas in or near all growth plates. This raises the question if there might be a secondary, unknown influence or factor. This suggestion is supported by multiple studies describing mixed cell populations with both mutant and wild cells in the cartilage of the osteochondromas ${ }^{59,91,92}$.

\section{Do OSTEOCHONDROMAS ESCAPE WOLFF'S LAW AND IF SO, HOW?}

Osteochondromas contain a bone marrow cavity continuing in the normal cavity of the long bone. The cap of the osteochondroma has the histological appearance of an epiphyseal growth plate with chondrocytes lined up in columns. The growth platelike lesion grows at an approximately 60-degree angle relative to the normal growth direction of the bone ${ }^{13}$. When osteochondromas arise there can be spontaneous regression ${ }^{93}$. This applies not only to naturally occurring osteochondromas but also to surgically created ones. Osteochondromas can be created by inverting a 60-degree span of the ring of LaCroix ${ }^{94}$. These surgically created osteochondromas disappear eventually due to spontaneous regression. One could expect all osteochondromas to disappear eventually, raising the question why the osteochondromas in $\mathrm{HMO}$ do not 


\section{Chapter 2}

resorb or regress, according to Wolff's law. Possibly, the answer lies not only in the osteochondromas itself, but also in the surroundings of the osteochondromas. The osteochondroma is covered with periosteum. This periosteal layer also covers the cartilaginous top. This layer consists of undifferentiated cells overlying the top of the osteochondroma. In culture they yield a rapidly proliferating homogenous population of fibroblast-like cells. These cells express FGF9, FGFR3, and collagen type Ila ${ }^{95}$, possibly influencing the cartilage cell in the top that resembles epiphyseal cells and may also have a similar function. The top of the osteochondroma then behaves like regular endochondral bone with active remodelling. Trebicz-Geffen et al. assessed surgically created osteochondromas and found a lack of FGF receptor 3 (FGFR3), and down-regulated Indian hedgehog ${ }^{96}$. Perhaps the presence of the epiphyseal-like chondrocyt carrying cap and the presence of growth regulatory factors such as FGF and Ihh coming from the covering layer gives active regeneration of the osteochondromas which shields them from resorption due to remodeling, explaining how the osteochondroma is constructed by the cartilaginous cells in the top and simultaneously broken down by the remodelling.

\section{WHAT IS THE PLACE OF ORIGIN OF OSTEOCHONDROMAS?}

Although we know that most osteochondromas are found in the metaphysis under the periosteum we still do not know where they originate. To find the place of origin, we first zoom in on the cellular marker expression. As mentioned, associations have been found between the epiphysis and osteochondromas, for example the proliferative zone resembling chondrocytes in osteochondromas stain positive for $\mathrm{PCNA}^{80}$. PCNA is a specific marker for S-phase cells, showing that these proliferative zone resembling cells indeed preserve their proliferative character. The cartilage cap of osteochondromas does not significantly thicken, which indicates that the proliferative cells undergo hypertrophy and the osteochondroma does retain a rudimentary epiphyseal function. Other similarities are found when using the proliferative marker Ki-67. Both the osteochondroma and the normal growth plate stained positive in equal measures, showing according to Huch et al. that the osteochondromas and the normal growth plate shared similar proliferation 
capacity $^{97}$. Benoist-Lasselin et al.proved growth plate phenotype by staining the osteochondromas positive for cartilage specific collagen type II, and hypertrophic zone specific marker collagen type $X^{80}$. The above makes the epiphyseal disc as a place of origin likely.

Looking at the histopathologic studies of very young patients, the earliest lesions are shown as a micro osteochondromas within the periosteum adjacent to the normal physis. This suggests the groove of Ranvier as a possible place of origin. This idea that the origin of the osteochondromas is the groove of Ranvier is supported by the fact that the osteochondromas always grows close to the epiphysis but never in it ${ }^{98-100}$. Other histopathologic studies as the study by Milgram et al. in 1983 showed that the osteochondromas are derived from aberrant cartilaginous epiphyseal growth plate tissue, which proliferates autonomously and separates from the normal growth plate near its edge. The aberrant tissue remains in a subperiosteal location, where it either disappears or proliferates ${ }^{15}$. This is bolstered by the finding that redistribution of Ihh from growth plate to perichondrium leads to ectopic cartilage formation ${ }^{101}$. This is further supported by studies conducted in mouse models of $\mathrm{HMO}$ that indicated ablation of Ext1 in growth plate chondrocytes leads to formation of ectopic cartilage around the epiphyses, not in it $^{91,92,102}$.

Looking at the research presented above, the growth plate seems to be the best possible place of origin of the osteochondromas both in cellular type as in cellular function of the chondrocytes found in osteochondromas.

\section{HistoricAl PERSPECTIVE ON THE theORIES ABOUT THE FORMATION OF OSTEOCHONDROMAS IN HMO}

In the earliest publications at the beginning of the 20st century the osteochondroma was believed to arise from an erroneous differentiation of cells in the periosteum ${ }^{103}$. However, many years later evidence showed that the perichondrium and the bony stalk were not of clonal origin, making it an unlikely pathophysiological source ${ }^{82}$. At the end of the 20st century, based on the observation that exposure to radiation could induce solitary osteochondroma formation, the link with DNA damage was made. The "loss to follow-up" was then postulated as a mechanism to explain the 


\section{Chapter 2}

formation. Researchers thought DNA damage to be a likely cause of diminished gene expression. Both in solitary and multiple, or familial, osteochondromas loss of heterozygosity $(\mathrm{LOH})$ of the EXT1 gene has been shown ${ }^{56,104,105}$. This suggests a common pathophysiological mechanism, which seems plausible based on the extensive similarities in morphology. But laboratory results showed that while $\mathrm{LOH}$ can occur in osteochondromas, and can induce their formation, it is not a consistent and thus necessary step in their development. This led to the search for a different explanation as to how gene expression can be down regulated. It seems obvious that since the EXT genes function in unison, their expression would also be regulated in a synchronized fashion. As the EXT genes are only expressed in specific zones of the normal epiphyseal disc, it might be that their expression is induced by differentiation. This defective differentiation is shown by the partial or absent signalling pathways in osteochondromas ${ }^{13}$. With the knowledge of the DNA blueprint in the 21st century, mouse, rat and zebra fish models were introduced. Many different authors then described the different pathways such as the defective Ihh signal and the Ihh-PTHrP feedback malfunctioning. Jones et al. in 2003 postulated a theory that an islet of EXT-/- chondrocytes would produce a defective Ihh signal, preventing the perichondrium from osteoblastic differentiation. Islet of chondrocytes differentiate and form an internal growth plate forming an osteochondroma $^{79}$. This theory emphasises the importance of the perichondrium, but does not offer an explanation on how the chondrocytes gain their proliferative capacity nor does it incorporate the evidence of abnormal osteoblastic and hypertrophic marker expression in osteochondroma chondrocytes.

Other evidence showed that the cartilage cap of an immature osteochondromas is of clonal origin and therefore of neoplastic nature ${ }^{56,104-106}$. This makes the cartilage cap the likelier intermediate for osteochondroma development and the growth plate the most likely source of pathological chondrocytes ${ }^{81}$. Currently different authors believe osteochondromas should be approached more like a derailed growth plate $^{4,59,80,97}$. The growth-plate-like structure of the osteochondromas seems to be fed by a reservoir of proliferative zone resembling chondrocytes, accompanied by hypertrophic zone resembling chondrocytes, which quite possibly are the differentiation products of the proliferative resembling cells ${ }^{107}$.

Now that the causative gene mutations have been clarified and the site of origin 
might be found, it is a matter of finding the pathophysiological "missing" link that brings us from gene mutation to osteochondroma formation. Knowing that the general genetic defect does not induce osteochondromas near all growth plates, the search for other explanations began. Different models were therefore introduced, such as Knudson's two hit model: the neoplastic theory ${ }^{82,108}$. It postulates that EXT null chondrocytes might lose their tumour suppressor function through loss of heterozygosity thereby causing osteochondromas ${ }^{58,99,109-112}$.

In summary, the well-preserved morphology of osteochondromas seems to entail a highly regulated process, deregulated in a highly consistent manner. The differentiation process is not of a pathological nature in itself but steered in the wrong direction. It can therefore count on a highly constant and regulated physiological response, also explaining the constant morphology. The EXT genes are only expressed throughout the proliferating and hypertrophic zone of the epiphyseal disc. Pathological derailment is likely to start as EXT heterozygous chondrocytes express less heparan sulphate. As heparan sulphate is essential to membrane bound and extracellular proteoglycans optimizing signalling transduction and gradient formation of several premier epiphyseal-signalling pathways, it seems apparent that part of the solution ought to be found in this aspect.

Possibly a malformation in gradient formation and signalling exposes chondrocytes to a unique dysphysiological morphogenic "cocktail". To understand the exact cocktail these chondrocytes are subjected to, more work is needed. 


\section{Chapter 2}

\section{CONCLUSION}

This review summarizes current knowledge about HMO. The pathophysiology and genetics of the EXT genes and their possible role in heparan sulphate biosynthesis are described. The theories about the growth of osteochondromas are analysed, finding clues in the mutations in either EXT1 or EXT2 that lead to the absence or reduction of HS. This seems to disturb the major regulatory systems of epiphyseal growth and of osteochondromas. The question on how osteochondromas escape Wolff's law is highlighted referring to the periostal layer and the osteochondromas proper growth plate. The presence of this epiphyseal like chondrocyt caring cap might give osteochondromas the possibility to regenerate and that might shield them from being resorpted due to remodelling. This active chondrocytic cap on the osteochondroma might be due to the place of origin of the cells that could very well be the growth plate. Thus also explaining their growth capacity in harmony with the growth of the patient. Because the general gene defect does not account for the difference in penetration of the disease in patients, further studies might be focussed on the search for multiple causes or defects. 


\section{REFERENCES}

1. Khurana J, A.-K.F.a.B.J, Osteochondroma (2002) World Health Organization Classification of Tumours. Pathology and Genetics of Tumours of Soft Tissue and Bone: 234-236.

2. Bovee JVMG (2002) HP Osteochondroma. World Health Organization Classification of Tumours. Pathology and Genetics of Tumours of Soft Tissue and Bone.

3. Schmale GA, Conrad EU 3rd, Raskind WH (1994) The natural history of hereditary multiple exostoses.J Bone Joint Surg Am 76: 986-992.

4. Kitsoulis P, Galani V, Stefanaki K, Paraskevas G, Karatzias G, et al. (2008) Osteochondromas: review of the clinical, radiological and pathological features.In Vivo 22: 633-646.

5. Bovée JV (2008) Multiple osteochondromas.Orphanet J Rare Dis 3: 3

6. Legeai-Mallet L, Munnich A, Maroteaux P, Le Merrer M (1997) Incomplete penetrance and expressivity skewing in hereditary multiple exostoses.Clin Genet 52: 12-16.

7. Hecht JT, Hall CR, Snuggs M, Hayes E, Haynes R, et al. (2002) Heparan sulfate abnormalities in exostosis growth plates.Bone 31: 199-204.

8. Bottner F, Rodl R, Kordish I, Winklemann W, Gosheger G, et al. (2003) Surgical treatment of symptomatic osteochondroma. A three- to eight-year follow-up study.J Bone Joint Surg Br 85: 1161-1165.

9. Darilek S, Wicklund C, Novy D, Scott A, Gambello M, et al. (2005) Hereditary multiple exostosis and pain.J PediatrOrthop 25: 369-376.

10. Wicklund CL, Pauli RM, Johnston D, Hecht JT (1995) Natural history study of hereditary multiple exostoses.Am J Med Genet 55: 43-46.

11. Carpintero P, León F, Zafra M, Montero M, Berral FJ (2003) Fractures of osteochondroma during physical exercise.Am J Sports Med 31: 1003-1006.

12. Hosalkar H, Greenberg J, Gaugler RL, Garg S, Dormans JP (2007) Abnormal scarring with keloid formation after osteochondroma excision in children with multiple hereditary exostoses.J PediatrOrthop 27: 333-337.

13. Vanhoenacker FM, Van Hul W, Wuyts W, Willems PJ, De Schepper AM (2001) Hereditary multiple exostoses: from genetics to clinical syndrome and complications.Eur J Radiol 40: 208-217.

14. Pannier S, Legeai-Mallet L (2008) Hereditary multiple exostoses and enchondromatosis.Best Pract Res ClinRheumatol 22: 45-54. 


\section{Chapter 2}

15. Milgram JW (1983) The origins of osteochondromas and enchondromas. A histopathologic study.ClinOrthopRelat Res : 264-284.

16. Chin KR, Kharrazi FD, Miller BS, Mankin HJ, Gebhardt MC (2000) Osteochondromas of the distal aspect of the tibia or fibula. Natural history and treatment.J Bone Joint Surg Am 82: 1269-1278.

17. Noonan KJ, Feinberg JR, Levenda A, Snead J, Wurtz LD (2002) Natural history of multiple hereditary osteochondromatosis of the lower extremity and ankle.J PediatrOrthop 22: 120124.

18. Hennekam RC (1991) Hereditary multiple exostoses.J Med Genet 28: 262-266.

19. Nawata K, Teshima R, Minamizaki T, Yamamoto K (1995) Knee deformities in multiple hereditary exostoses. A longitudinal radiographic study.ClinOrthopRelat Res : 194-199.

20. Leone NC, Shupe JL, Gardner EJ, Millar EA, Olson AE, et al. (1987) Hereditary multiple exostosis. A comparative human-equine-epidemiologic study.J Hered 78: 171-177.

21. Legeai-Mallet L, Rossi A, Benoist-Lasselin C, Piazza R, Mallet JF, et al. (2000) EXT 1 gene mutation induces chondrocyte cytoskeletal abnormalities and defective collagen expression in the exostoses.J Bone Miner Res 15: 1489-1500.

22. Woertler K, Lindner N, Gosheger G, Brinkschmidt C, Heindel W (2000) Osteochondroma: MR imaging of tumor-related complications.EurRadiol 10: 832-840.

23. Gordon SL, Buchanan JR, Ladda RL (1981) Hereditary multiple exostoses: report of a kindred.J Med Genet 18: 428-430.

24. Peterson HA (1989) Multiple hereditary osteochondromata.ClinOrthopRelat Res : 222-230.

25. Wise CA, Clines GA, Massa H, Trask BJ, Lovett M (1997) Identification and localization of the gene for EXTL, a third member of the multiple exostoses gene family.Genome Res 7: 1016.

26. Wuyts W, Van Hul W, Hendrickx J, Speleman F, Wauters J, et al. (1997) Identification and characterization of a novel member of the EXT gene family, EXTL2.Eur J Hum Genet 5: 382389.

27. Van Hul W, Wuyts W, Hendrickx J, Speleman F, Wauters J, et al. (1998) Identification of a third EXT-like gene (EXTL3) belonging to the EXT gene family.Genomics 47: 230-237.

28. White SJ, Vink GR, Kriek M, Wuyts W, Schouten J, et al. (2004) Two-color multiplex ligation-dependent probe amplification: detecting genomic rearrangements in hereditary multiple exostoses. Hum Mutat 24: 86-92. 
29. Signori E, Massi E, Matera MG, Poscente M, Gravina C, et al. (2007) A combined analytical approach reveals novel EXT1/2 gene mutations in a large cohort of Italian multiple osteochondromas patients. Genes Chromosomes Cancer46: 470-477.

30. Cook A, Raskind W, Blanton SH, Pauli RM, Gregg RG, et al. (1993) Genetic heterogeneity in families with hereditary multiple exostoses.Am J Hum Genet 53: 71-79.

31. Wu YQ, Heutink P, de Vries BB, Sandkuijl LA, van den Ouweland AM, et al. (1994) Assignment of a second locus for multiple exostoses to the pericentromeric region of chromosome 11. Hum Mol Genet 3: 167-171.

32. Raskind WH, Conrad EU 3rd, Matsushita M, Wijsman EM, Wells DE, et al. (1998) Evaluation of locus heterogeneity and EXT1 mutations in 34 families with hereditary multiple exostoses. Hum Mutat 11: 231-239.

33. Le Merrer M, Legeai-Mallet L, Jeannin PM, Horsthemke B, Schinzel A, et al. (1994) A gene for hereditary multiple exostoses maps to chromosome 19p. Hum Mol Genet 3: 717-722.

34. Porter DE, Lonie L, Fraser M, Dobson-Stone C, Porter JR, et al. (2004) Severity of disease and risk of malignant change in hereditary multiple exostoses. A genotype-phenotype study.J Bone Joint Surg Br 86: 1041-1046.

35. Jäger M, Westhoff B, Portier S, Leube B, Hardt K, et al. (2007) Clinical outcome and genotype in patients with hereditary multiple exostoses.J Orthop Res 25: 1541-1551.

36. Francannet C, Cohen-Tanugi A, Le Merrer M, Munnich A, Bonaventure J, et al. (2001) Genotype-phenotype correlation in hereditary multiple exostoses.J Med Genet 38: 430434.

37. Carroll KL, Yandow SM, Ward K, Carey JC (1999) Clinical correlation to genetic variations of hereditary multiple exostosis.J PediatrOrthop 19: 785-791.

38. Alvarez CM, De Vera MA, Heslip TR, Casey B (2007) Evaluation of the anatomic burden of patients with hereditary multiple exostoses.ClinOrthopRelat Res 462: 73-79.

39. Alvarez C, Tredwell S, De Vera M, Hayden M (2006) The genotype-phenotype correlation of hereditary multiple exostoses.Clin Genet 70: 122-130.

40. Stickens D, Zak BM, Rougier N, Esko JD, Werb Z (2005) Mice deficient in Ext2 lack heparan sulfate and develop exostoses.Development 132: 5055-5068.

41. Lind T, Tufaro F, McCormick C, Lindahl U, Lidholt K (1998) The putative tumor suppressors EXT1 and EXT2 are glycosyltransferases required for the biosynthesis of heparan sulfate.J BiolChem 273: 26265-26268. 


\section{Chapter 2}

42. Kitagawa H, Shimakawa H,Sugahara K (1999) The tumor suppressor EXT-like gene EXTL2 encodes an alpha1, 4-N-acetylhexosaminyltransferase that transfers $\mathrm{N}$-acetylgalactosamine and $\mathrm{N}$-acetylglucosamine to the common glycosaminoglycan-protein linkage region. The key enzyme for the chain initiation of heparan sulfate. J BiolChem 274:13933-13937.

43. McCormick C, Leduc Y, Martindale D, Mattison K, Esford LE, et al. (1998) The putative tumour suppressor EXT1 alters the expression of cell-surface heparan sulfate.Nat Genet 19: 158-161.

44. Senay C, Lind T, Muguruma K, Tone Y, Kitagawa H, et al. (2000) The EXT1/EXT2 tumor suppressors: catalytic activities and role in heparan sulfate biosynthesis.EMBO Rep 1: 282286.

45. Kobayashi S, Morimoto K, Shimizu T, Takahashi M, Kurosawa H, et al. (2000) Association of EXT1 and EXT2, hereditary multiple exostoses gene products, in Golgi apparatus. BiochemBiophys Res Commun 268: 860-867.

46. Sugahara K, Kitagawa H (2000) Recent advances in the study of the biosynthesis and functions of sulfated glycosaminoglycans.CurrOpinStructBiol 10: 518-527.

47. Kim BT, Kitagawa H, Tamura J, Saito T, Kusche-Gullberg M, et al. (2001) Human tumor suppressor EXT gene family members EXTL1 and EXTL3 encode alpha 1,4- Nacetylglucosaminyltransferases that likely are involved in heparan sulfate/ heparin biosynthesis.ProcNatlAcadSci U S A 98: 7176-7181.

48. Kim BT, Kitagawa H, Tanaka J, Tamura J, Sugahara K (2003) In vitro heparan sulfate polymerization: crucial roles of core protein moieties of primer substrates in addition to the EXT1-EXT2 interaction.J BiolChem 278: 41618-41623.

49. Lin X, Wei G, Shi Z, Dryer L, Esko JD, et al. (2000) Disruption of gastrulation and heparan sulfate biosynthesis in EXT1-deficient mice.DevBiol 224: 299-311.

50. Pedersen LC, Tsuchida K, Kitagawa H, Sugahara K, Darden TA, et al. (2000) Heparan/chondroitin sulfate biosynthesis. Structure and mechanism of human glucuronyltransferase I.J BiolChem 275: 34580-34585.

51. Wiggins CA, Munro S (1998) Activity of the yeast MNN1 alpha-1,3-mannosyltransferase requires a motif conserved in many other families of glycosyltransferases.ProcNatlAcadSci U S A 95: 7945-7950.

52. Busch C, Hofmann F, Selzer J, Munro S, Jeckel D, et al. (1998) A common motif of eukaryotic glycosyltransferases is essential for the enzyme activity of large clostridialcytotoxins.J BiolChem 273: 19566-19572. 
53. Munro S, Freeman M (2000) The notch signalling regulator fringe acts in the Golgi apparatus and requires the glycosyltransferase signature motif DXD.CurrBiol 10: 813-820.

54. Li J, Rancour DM, Allende ML, Worth CA, Darling DS, et al. (2001) The DXD motif is required for GM2 synthase activity but is not critical for nucleotide binding.Glycobiology 11: 217-229.

55. McCormick C, Duncan G, Tufaro F (1999) New perspectives on the molecular basis of hereditary bone tumours.Mol Med Today 5: 481-486.

56. Bovée JV, Cleton-Jansen AM, Wuyts W, Caethoven G, Taminiau AH, et al. (1999) EXTmutation analysis and loss of heterozygosity in sporadic and hereditary osteochondromas and secondary chondrosarcomas.Am J Hum Genet 65: 689-698.

57. Duncan G, McCormick C, Tufaro F (2001) The link between heparan sulfate and hereditary bone disease: finding a function for the EXT family of putative tumor suppressor proteins.J Clin Invest 108: 511-516.

58. Zak BM, Crawford BE, Esko JD (2002) Hereditary multiple exostoses and heparan sulfate polymerization.BiochimBiophysActa 1573: 346-355.

59. Huegel J, Mundy C, Sgariglia F, Nygren P, Billings PC, Yamaguchi Y, Koyama E, Pacifici M. (2013) Perichondrium phenotype and border function are regulated by Ext1 and heparan sulfate in developing long bones: a mechanism likely deranged in Hereditary Multiple Exostoses.DevBiol 377: 100-112.

60. Selleck SB (2000) Proteoglycans and pattern formation: sugar biochemistry meets developmental genetics. Trends Genet 16: 206-212.

61. Takada T, Katagiri T, Ifuku M, Morimura N, Kobayashi M, Hasegawa K, Ogamo A, Kamijo R. (2003) Sulfated polysaccharides enhance the biological activities of bone morphogenetic proteins.J BiolChem 278: 43229-43235.

62. Lander AD, Selleck SB (2000) The elusive functions of proteoglycans: in vivo veritas.J Cell Biol 148: 227-232.

63. Perrimon N, Bernfield M (2000) Specificities of heparansulphate proteoglycans in developmental processes. Nature 404: 725-728.

64. Baeg GH, Lin X, Khare N, Baumgartner S, Perrimon N (2001) Heparan sulfate proteoglycans are critical for the organization of the extracellular distribution of Wingless.Development 128: 87-94. 


\section{Chapter 2}

65. Takei Y, Ozawa Y, Sato M, Watanabe A, Tabata T (2004) Three Drosophila EXT genes shape morphogen gradients through synthesis of heparan sulfate proteoglycans. Development 131: 73-82.

66. Bellaiche Y, The I, Perrimon N (1998) Tout-velu is a Drosophila homologue of the putative tumour suppressor EXT-1 and is needed for Hh diffusion.Nature 394: 85-88.

67. The I, Bellaiche Y, Perrimon N (1999) Hedgehog movement is regulated through tout veludependent synthesis of a heparan sulfate proteoglycan.Mol Cell 4: 633-639.

68. Toyoda H, Kinoshita-Toyoda A, Fox B, Selleck SB (2000) Structural analysis of glycosaminoglycans in animals bearing mutations in sugarless, sulfateless, and tout-velu. Drosophila homologues of vertebrate genes encoding glycosaminoglycan biosynthetic enzymes.J BiolChem 275: 21856-21861.

69. Reichsman F, Smith L, Cumberledge S (1996)Glycosaminoglycans can modulate extracellular localization of the wingless protein and promote signal transduction. J Cell Biol 135: 819-827

70. Karimian E, Chagin AS, Sävendahl L (2012) Genetic regulation of the growth plate.Front Endocrinol (Lausanne) 2: 113.

71. Emons J, Chagin AS, Sävendahl L, Karperien M, Wit JM (2011) Mechanisms of growth plate maturation and epiphyseal fusion. Horm Res Paediatr 75: 383-391.

72. Enomoto-Iwamoto M, Nakamura T, Aikawa T, Higuchi Y, Yuasa T, et al. (2000) Hedgehog proteins stimulate chondrogenic cell differentiation and cartilage formation.J Bone Miner Res 15: 1659-1668.

73. St-Jacques B, Hammerschmidt M, McMahon AP (1999) Indian hedgehog signaling regulates proliferation and differentiation of chondrocytes and is essential for bone formation.Genes Dev 13: 2072-2086.

74. Maeda Y, Nakamura E, Nguyen MT, Suva LJ, Swain FL, et al. (2007) Indian Hedgehog produced by postnatal chondrocytes is essential for maintaining a growth plate and trabecular bone.ProcNatlAcadSci U S A 104: 6382-6387.

75. Hirai T, Chagin AS, Kobayashi T, Mackem S, Kronenberg HM (2011) Parathyroid hormone/parathyroid hormone-related protein receptor signaling is required for maintenance of the growth plate in postnatal life.ProcNatlAcadSci U S A 108: 191-196.

76. Hellemans J, Coucke PJ, Giedion A, De Paepe A, Kramer P, et al. (2003) Homozygous mutations in $\mathrm{IHH}$ cause acrocapitofemoral dysplasia, an autosomal recessive disorder with cone-shaped epiphyses in hands and hips.Am J Hum Genet 72: 1040-1046. 
77. de Andrea CE, Prins FA, Wiweger MI, Hogendoorn PC (2011) Growth plate regulation and osteochondroma formation: insights from tracing proteoglycans in zebrafish models and human cartilage.J Pathol 224: 160-168.

78. Koziel L, Kunath M, Kelly OG, Vortkamp A (2004) Ext1-dependent heparan sulfate regulates the range of Ihh signaling during endochondral ossification.Dev Cell 6: 801-813.

79. Jones KB, Morcuende JA (2003) Of hedgehogs and hereditary bone tumors: re-examination of the pathogenesis of osteochondromas.lowa Orthop J 23: 87-95.

80. Benoist-Lasselin C, de Margerie E, Gibbs L, Cormier S, Silve C, et al. (2006) Defective chondrocyte proliferation and differentiation in osteochondromas of MHE patients.Bone 39: 17-26.

81. Hameetman L, Rozeman LB, Lombaerts M, Oosting J, Taminiau AH, Cleton-Jansen AM, Bovée JV, Hogendoorn PC. (2006) Peripheral chondrosarcoma progression is accompanied by decreased Indian Hedgehog signalling.J Pathol 209: 501-511.

82. Hameetman L, Szuhai K, Yavas A, Knijnenburg J, van Duin M, van Dekken H, Taminiau AH, Cleton-Jansen AM, Bovée JV, Hogendoorn PC. (2007) The role of EXT1 in nonhereditary osteochondroma: identification of homozygous deletions.J Natl Cancer Inst 99: 396-406.

83. Moser M, Patterson C (2005) Bone morphogenetic proteins and vascular differentiation: BMPing up vasculogenesis. ThrombHaemost 94: 713-718.

84. Zhang F, Qiu T, Wu X, Wan C, Shi W, Wang Y, Chen JG, Wan M, Clemens TL, Cao X. (2009) Sustained BMP signaling in osteoblasts stimulates bone formation by promoting angiogenesis and osteoblast differentiation.J Bone Miner Res 24: 1224-1233.

85. Storm EE, Kingsley DM (1999) GDF5 coordinates bone and joint formation during digit development.DevBiol 209: 11-27.

86. Baur ST, Mai JJ, Dymecki SM (2000) Combinatorial signaling through BMP receptor IB and GDF5: shaping of the distal mouse limb and the genetics of distal limb diversity.Development 127: 605-619.

87. Pizette S, Niswander L (2000) BMPs are required at two steps of limb chondrogenesis: formation of prechondrogenic condensations and their differentiation into chondrocytes.DevBiol 219: 237-249.

88. Minina E, Wenzel HM, Kreschel C, Karp S, Gaffield W, McMahon AP, Vortkamp A. (2001) $\mathrm{BMP}$ and $\mathrm{Ihh} / \mathrm{PTHrP}$ signaling interact to coordinate chondrocyte proliferation and differentiation.Development 128: 4523-4534. 


\section{Chapter 2}

89. Lazarus JE, Hegde A, Andrade AC, Nilsson O, Baron J (2007) Fibroblast growth factor expression in the postnatal growth plate.Bone 40: 577-586.

90. Bovée JV, van den Broek LJ, Cleton-Jansen AM, Hogendoorn PC (2000) Up-regulation of $\mathrm{PTHrP}$ and $\mathrm{Bcl}-2$ expression characterizes the progression of osteochondroma towards peripheral chondrosarcoma and is a late event in central chondrosarcoma.Lab Invest 80: 1925-1934.

91. Matsumoto K, Irie F, Mackem S, Yamaguchi Y (2010) A mouse model of chondrocytespecific somatic mutation reveals a role for Ext1 loss of heterozygosity in multiple hereditary exostoses.ProcNatlAcadSci U S A 107: 10932-10937.

92. Jones KB, Piombo V, Searby C, Kurriger G, Yang B, Grabellus F, Roughley PJ, Morcuende JA, Buckwalter JA, Capecchi MR, Vortkamp A, Sheffield VC. (2010) A mouse model of osteochondromagenesis from clonal inactivation of Ext1 in chondrocytes.ProcNatlAcadSci U S A 107: 2054-2059.

93. Yanagawa T, Watanabe H, Shinozaki T, Ahmed AR, Shirakura K, Takagishi K. (2001) The natural history of disappearing bone tumours and tumour-like conditions.ClinRadiol 56: 877-886.

94. Delgado E, Rodríguez JI, Rodríguez JL, Miralles C, Paniagua R (1987) Osteochondroma induced by reflection of the perichondrial ring in young rat radii.Calcif Tissue Int 40: 85-90.

95. Robinson D, Hasharoni A, Oganesian A, Sandell LJ, Yayon A, Nevo Z. (2001) Role of FGF9 and FGF receptor 3 in osteochondroma formation.Orthopedics 24: 783-787.

96. Trebicz-Geffen M, Nevo Z, Evron Z, Posternak N, Glaser T, Fridkin M, Kollander Y, Robinson D. (2003) The short-lived exostosis induced surgically versus the lasting genetic hereditary multiple exostoses.ExpMolPathol 74: 40-48.

97. Huch K, Mordstein V, Stöve J, Nerlich AG, Amholdt H, Delling G, Puhl W, Günther KP, Brenner RE. (2002) Expression of collagen type I, II, X and Ki-67 in osteochondroma compared to human growth plate cartilage.Eur J Histochem 46: 249-258.

98. Mansoor A, Beals RK (2007) Multiple exostosis: a short study of abnormalities near the growth plate.J PediatrOrthop B 16: 363-365.

99. Porter DE, Simpson AH (1999) The neoplastic pathogenesis of solitary and multiple osteochondromas.J Pathol 188: 119-125.

100. Hecht JT, Hayes E, Haynes R, Cole WG, Long RJ, Farach-Carson MC, Carson DD. (2005) Differentiation-induced loss of heparan sulfate in human exostosis derived chondrocytes.Differentiation 73: 212-221. 
101. Koyama E, Young B, Nagayama M, Shibukawa Y, Enomoto-Iwamoto M, Maeda Y, Lanske B, Song B, Serra R, Pacifici M. (2007) Conditional Kif3a ablation causes abnormal hedgehog signaling topography, growth plate dysfunction, and excessive bone and cartilage formation during mouse skeletogenesis. Development 134: 2159-2169.

102. Zak BM, Schuksz M, Koyama E, Mundy C, Wells DE, Yamaguchi Y, Pacifici M, Esko JD. (2011) Compound heterozygous loss of Ext1 and Ext2 is sufficient for formation of multiple exostoses in mouse ribs and long bones.Bone 48: 979-987.

103. Muller (1914)Uberhereditare multiple cartilaginareexostosen und ecchondrosen. BeitrPatholAnat57: 232.

104. Mertens F, Rydholm A, Kreicbergs A, Willén H, Jonsson K, Heim S, Mitelman F, Mandahl N. (1994) Loss of chromosome band 8q24 in sporadic osteocartilaginousexostoses.Genes Chromosomes Cancer 9: 8-12.

105. Bridge JA, Nelson M, Orndal C, Bhatia P, Neff JR (1998)Clonal karyotypic abnormalities of the hereditary multiple exostoses chromosomal loci 8q24.1 (EXT1) and 11p11-12 (EXT2) in patients with sporadic and hereditary osteochondromas. Cancer 82: 1657-1663.

106. Stickens D, Evans GA (1998) A sugar fix for bone tumours? Nat Genet 19: 110-111.

107. Nakase T, Myoui A, Shimada K, Kuriyama K, Joyama S, Miyaji T, Tomita T, Yoshikawa H. (2001) Involvement of BMP-2 signaling in a cartilage cap in osteochondroma.J Orthop Res 19: 1085-1088.

108. Knudson AG Jr (1971) Mutation and cancer: statistical study of retinoblastoma. ProcNatlAcadSci U S A 68: 820-823.

109. Hall CR, Cole WG, Haynes R, Hecht JT (2002) Reevaluation of a genetic model for the development of exostosis in hereditary multiple exostosis.Am J Med Genet 112: 1-5.

110. Ahn J, Lüdecke HJ, Lindow S, Horton WA, Lee B, Wagner MJ, Horsthemke B, Wells DE. (1995) Cloning of the putative tumour suppressor gene for hereditary multiple exostoses (EXT1).Nat Genet 11: 137-143.

111. Stickens D, Clines G, Burbee D, Ramos P, Thomas S, Hogue D, Hecht JT, Lovett M, Evans GA. (1996) The EXT2 multiple exostoses gene defines a family of putative tumour suppressor genes.Nat Genet 14: 25-32.

112. Wuyts W, Radersma R, Storm K, Vits L (2005) An optimized DHPLC protocol for molecular testing of the EXT1 and EXT2 genes 


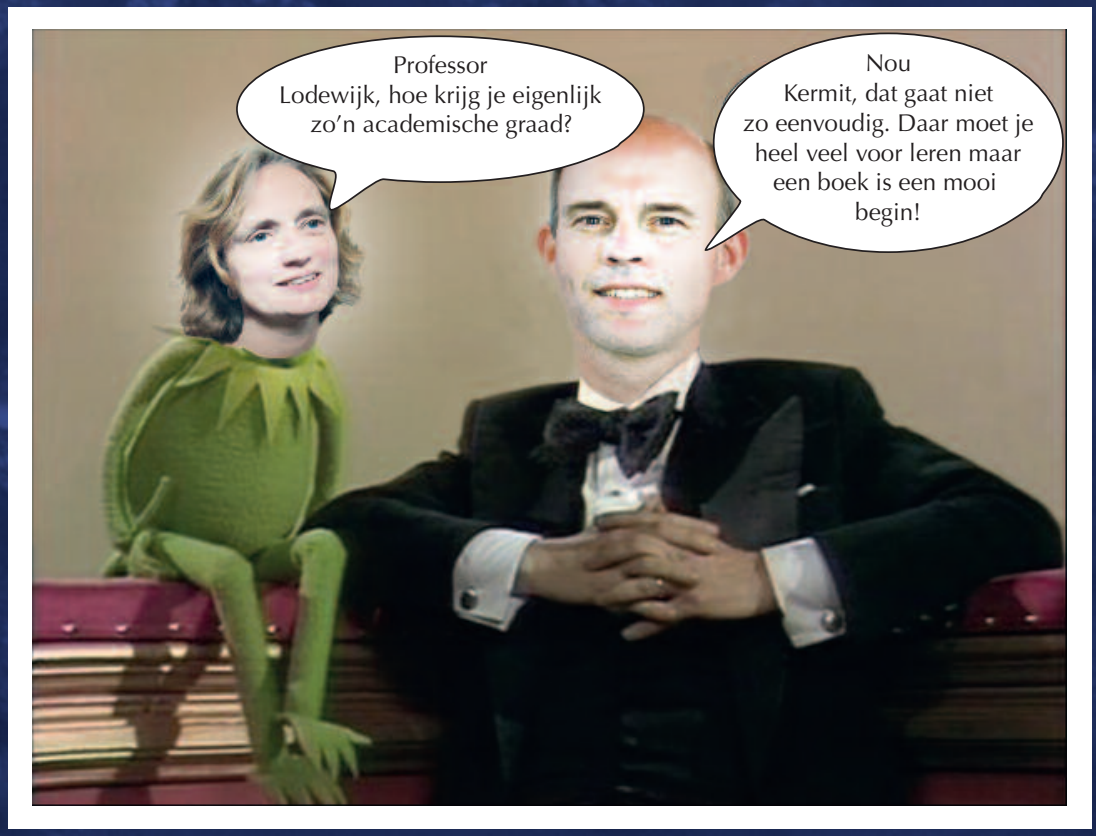




\section{CHAPTER THREE}

\section{Distal femoral osteochondromas in patients with Hereditary Multiple Osteochondromas, a longitudinal radiological assessment}

Heleen M. Staal, Wouter Nijhuis, Gerjon Hannink, M. Adhiambo Witlox, Arnard L. van der Zwan, S. John Ham, Chris J. Arts, Lodewijk W. van Rhijn.

International Journal of Research Studies in Biosciences (IIRSB) Volume 3, Issue 8, August 2015, PP 68-77 


\section{Chapter 3}

\section{AbStRACT}

Background: The nature of distal femoral osteochondromas in terms of size and position in patients with hereditary multiple osteochondromas ( $\mathrm{HMO}$ ) during skeletal growth has never been assessed before. This study was set up to address three specific aims: 1) to assess whether it is possible to reproducibly measure growth of osteochondromas on plain X-rays, 2) to assess which parameters best describe the relationship between growth of the femur and development of osteochondromas, and 3) to assess whether the osteochondromas' place of origin and its positional relation to the femur changes during skeletal growth.

Methods: We retrospectively reviewed X-rays of ten patients diagnosed with $\mathrm{HMO}$, with sixteen individual osteochondromas and measured the length of the femur from the fossa piriformis to the joint line of the knee, the distance from the base of the osteochondroma to the joint line of the knee, the base of the osteochondroma, the cross section of the femur at the most proximal part of the base and the length of the osteochondroma measured from top to base. Newly formed osteochondromas were registered. Two observers performed all measurements independently. The interobserver agreement was estimated using concordance correlation coefficients (CCC).

Results: The length of the femur (CCC $0.98(95 \% \mathrm{Cl} 0,97-0,99))$ and the height of the osteochondroma (CCC 0.87 ) could be measured with high reproducibility between individual observers. Measurement of the femoral cross section and the base of the osteochondroma (CCC 0.59 and 0.38 respectively) and the distance of the osteochondroma to the joint line of the knee (CCC 0.43) resulted in low reproducibility. The height of the osteochondroma and the distance to the joint line of the knee increased over time during growth. No newly formed osteochondromas were detected proximal to the existing osteochondromas in the distal femur.

Conclusions: The length of the femur and the height of the osteochondromas could be reliably measured on plain X-rays. No new osteochondroma formed proximal to the already existing ones, indicating that they are formed in or close to the epiphyseal line. It seems likely that the osteochondromas are formed at or near the epiphyses during growth and stay in their place of origin, as the femur grows more distal, resulting in a more proximal position of the osteochondromas over time.

Level of Evidence: IV (retrospective case series) 


\section{INTRODUCTION}

Hereditary Multiple Osteochondromas (HMO) or Hereditary Multiple Exostoses (HME) is an autosomal dominant disorder known for characteristic growth of osteochondromas ${ }^{1-4}$. According to the definition of the World Health Organization (WHO), osteochondromas are cartilage-capped bony outgrowths on the external surface of long bones, consisting of a marrow cavity that is continuous with that of the underlying bone $e^{2,5,6}$.

For a diagnosis of $\mathrm{HMO}$ to be made, two or more lesions must be radiographically identified. Osteochondromas typically occur around the metaphysis of long bones and have an incidence of approximately 1 in $50.000^{7,8}$. The onset of HMO may be at any time between early childhood (2-3 years) and puberty with the majority affected during the first decade of life ${ }^{9}$. Clinically short stature is considered as a common feature of $\mathrm{HMO}$, with the majority of affected individuals being below average height but within a normal range $\mathrm{e}^{4,10,11}$.

Osteochondromas may be sessile or pedunculated and can vary in number and size. They are usually painless and come to clinical attention for cosmetic reasons. Nevertheless, large osteochondromas can cause a variety of clinical problems such as pain, bursa formation, fracture after local trauma, or snapping may occur when a large muscle moves over the top of the osteochondroma ${ }^{12}$. In addition, osteochondromas on the lower limb can cause various deformities. The most common findings are genu valgum and lower limb length discrepancy ${ }^{13,14}$. The way in which osteochondromas influence these growth deformities is not clear. Osteochondromas develop when the epiphyseal plate is not yet closed ${ }^{9,15}$ and even though the pathogenesis of $\mathrm{HMO}$ has not been completely elucidated, the most adopted hypothesis suggests that the osteochondromas develop in the epiphyseal plate. Nests of cartilage being misplaced fragments of cartilage around the epiphyseal line become isolated on the surface of the metaphysis, proliferate, and form the osteochondroma ${ }^{16-19}$. The periosteum, which is incomplete at the sites of these cartilaginous nests, fails to remodel the metaphysis in a normal manner ${ }^{16}$. If the osteochondroma is formed near the epiphysis, we can assume that the growth deformities arise because osteochondromas influence the epiphysis either in a mechanical or a biochemical manner. Because most osteochondromas in $\mathrm{HMO}$ 


\section{Chapter 3}

occur in the distal femur, this is a logical site to investigate the influence of osteochondromas on growth. The nature of distal femoral osteochondroma in terms of size and position during skeletal growth has to our knowledge not been assessed until now. Therefore the aim of this study is to analyse the development of the osteochondromas of the distal femur and to compare their growth to the growth of the distal femur. Since osteochondromas are formed near the epiphysis, it is hypothesized that the osteochondromas form near the epiphysis and continue to grow while the femur is growing and therefore the osteochondromas stay in their place of origin. In this manner new ostoechondromas can only form closer to the epiphyseal plate than already existing ones.

This study was set up to address three specific aims: 1 ) to assess whether it is possible to reproducibly measure growth of osteochondromas on plain X-rays, 2) to assess which parameters best describe the relationship between growth of the femur and development of osteochondromas, and 3) to assess whether the osteochondromas' place of origin and its positional relation to the femur changes during skeletal growth.

\section{Patients AND Methods}

Ten patients were selected for this study from a population of fifty-three patients with $\mathrm{HMO}$ under twenty years old from a $\mathrm{HMO}$ study population of two medical centres in the Netherlands (OLVG, Amsterdam and MUMC+, Maastricht). Inclusion criteria were at least 3 calibrated anterior-posterior (AP) long standing X-rays of either one leg or both, taken at different time points. Two or more of these X-rays should been taken when the epiphysis was still open. Furthermore, patients should have at least one lateral knee radiograph in order to determine the direction of the osteochondroma in a three dimensional plane. Immeasurable osteochondroma or technical improper X-ray resolution were considered exclusion criteria. The ten selected patients (12 legs) accounted for 16 osteochondromas that were included in this study. The radiological measurements were performed by two clinicians (HS and WN; orthopaedic surgeons) independently from each other. All measurements were performed on digital calibrated x-rays with digital measuring software. All X-rays were successively analysed per patient. 
The following measurements were performed as shown in Figure $1 \mathrm{a}$ and $1 \mathrm{~b}$ (Figure 1ab):

A. Length of the femur from the fossa piriformis to the joint line of the knee in $(\mathrm{mm})$

B. Distance between base of the osteochondroma and joint line of the knee $(\mathrm{mm})$

C. Cross-section of femur (outside of medial to lateral cortex, perpendicular to the joint line) at the most proximal part of the base $(\mathrm{mm})$.

D. Length of the base of the osteochondroma $(\mathrm{mm})$

E. Height of the osteochondroma from the centre of the base to the top $(\mathrm{mm})$

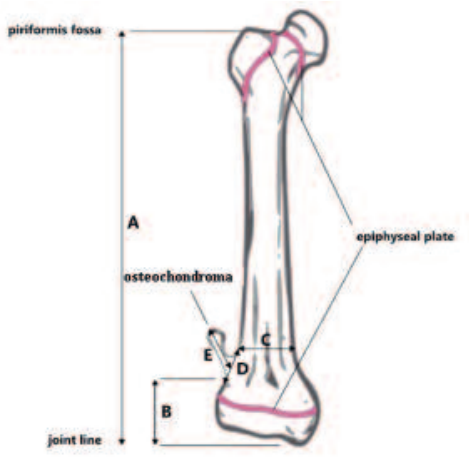

Figure 1a: Schematic figure of the measured parameters on the X-ray of a femur with a distal osteochondroma: A: Length of the femur from the fossa piriformis to the joint line of the knee. B: Distance between base of the osteochondroma and joint line of the knee. C: Cross section femur at the most proximal part of the base: D: Length of the base. E: Length of the osteochondroma.

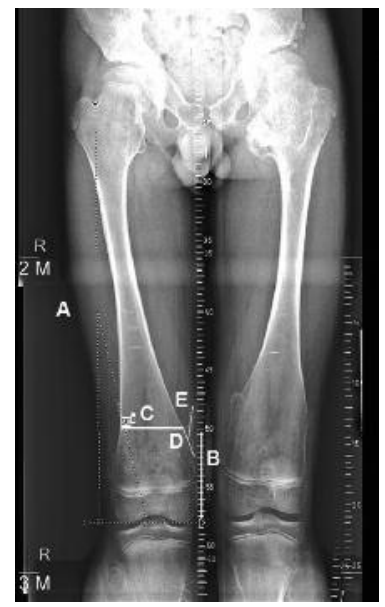

Figure 1b: Representation of the same measured parameters on the X-ray of a femur with a distal osteochondroma.

The length of the femur (A) was measured from the fossa piriformis to the joint line of the knee in order to measure only the growth of the distal epiphyses. The distance of the osteochondroma base to the joint line of the knee (B) was measured to determine the location of the osteochondroma 


\section{Chapter 3}

on the femur during growth. Besides the measurements A-E, all radiographs were checked for occurrence of new osteochondromas.

\section{Statistical analysis}

Differences in measurements (A-E) between observers were plotted against the average of observers using modified Bland-Altman plots. The limits of agreement (prediction limits for the differences) were estimated using a variance components model in which replicates were linked within subjects across observers.

Subsequently, for each measurement (A-E) the inter-observer agreement was estimated using concordance correlation coefficients. The concordance correlation coefficient (CCC) is one of the most common approaches to assess agreement where the design of the data involves repeated measurements on subjects by multiple observers. The CCC is a standardized coefficient that takes values between -1 and 1 , where -1 means perfect disagreement, 0 translates to an independence situation (all the readings are at random), and 1 indicates perfect agreement ${ }^{20,21}$. Variance components were used to calculate the CCC. Variance and error components were estimated from linear mixed models in which for all sources of variation were accounted for. Subject, observer, subject-observer interaction, and subject-age interaction were treated as random factors. CCCs are reported as point estimates with their 95\% confidence intervals. The statistical analyses were performed using $\mathrm{R}$ version 3.0.2 with packages 'MethComp' and 'cccrm' ${ }^{22-24}$.

\section{ResUlts}

In 10 patients (12 legs), 16 osteochondromas were identified and measured. There were 6 males and 4 females. The average age was 11.6 (range 10-14) at the start of measurement. Mean interval between the first and last measurements was 44.4 months (range 22-64 months). In one patient (patient no. 6) the cross section of the femur could not be measured due to an overprojection of another osteochondroma on the overlying cortex. 
A

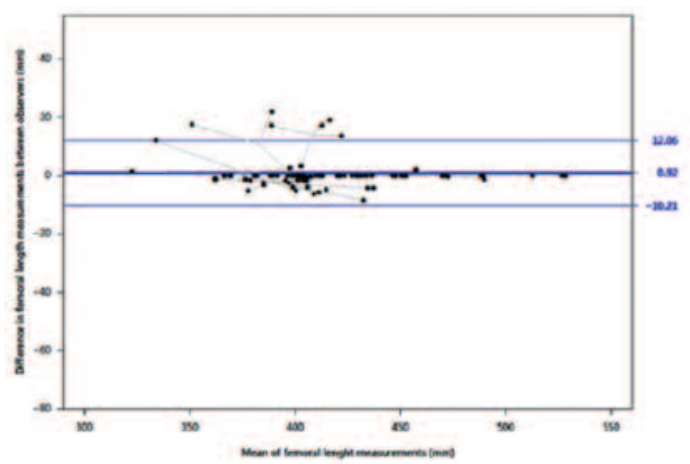

B

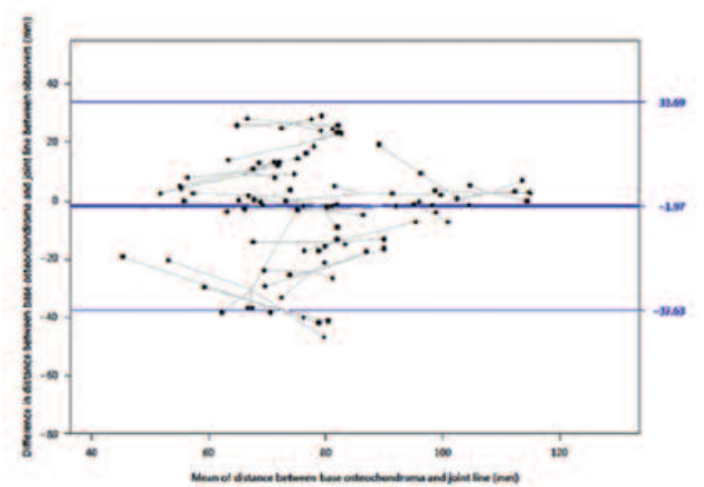

C

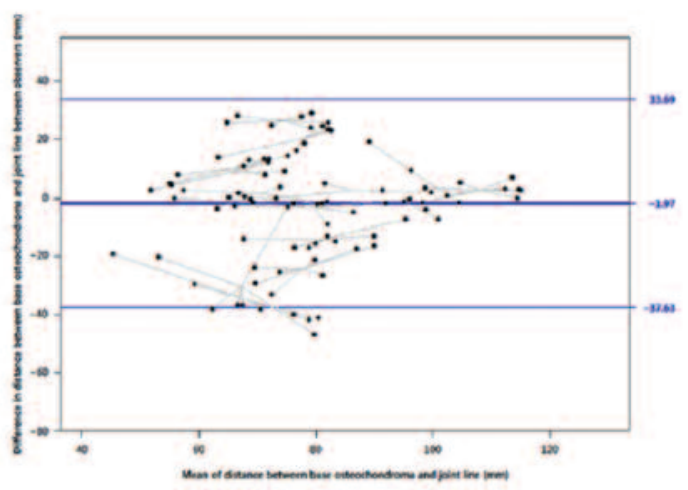

Figure 2: Modified Bland-Altman plots measurments A to C. Differences in measurements between observers were plotted against the average of observers. Measurements in time within an osteochondroma are linked with grey lines. Mean differences between observers with 95\% confidence limits are shown in blue. 


\section{Chapter 3}

D

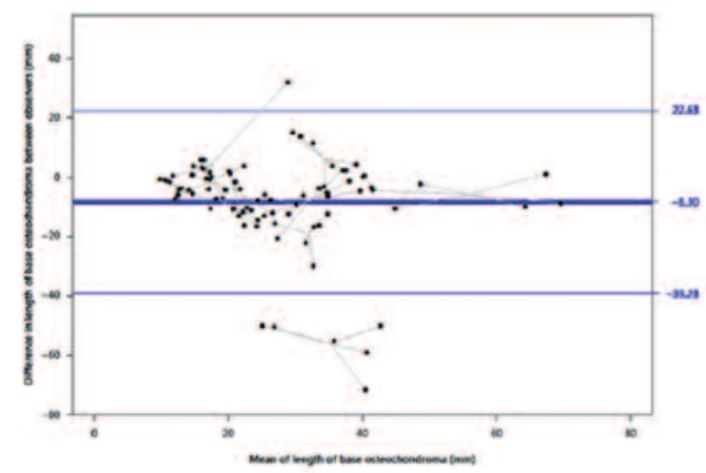

$\mathrm{E}$

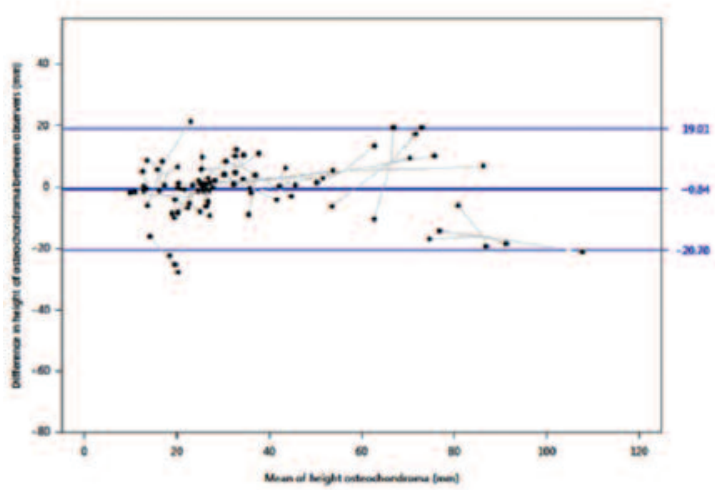

Figure 2: Modified Bland-Altman plots measurments D and E. Differences in measurements between observers were plotted against the average of observers. Measurements in time within an osteochondroma are linked with grey lines. Mean differences between observers with $95 \%$ confidence limits are shown in blue.

Differences in measurements (A-E) between observers were plotted against the average of observers using modified Bland-Altman plots (Figures 2A-E). The interobserver CCCs for total length of the femur and height of the osteochondroma were 0.99 (95\% $\mathrm{Cl} 0.97-0.99)$ and $0.87(95 \% \mathrm{Cl} 0.70-0.94)$, respectively, indicating a high inter-observer reliability for both measurements. Inter-observer CCCs for cross section of the femur at the most proximal part, the base of the osteochondroma, and distance from the base of the osteochondroma to the joint line of the femur indicated low inter-observer reliability $(0.59(95 \% \mathrm{Cl} 0.23-0.81), 0.38(95 \% \mathrm{Cl} 0.00-0.66)$, and $0.43(95 \% \mathrm{Cl}-0.01-0.73)$, respectively). 
A
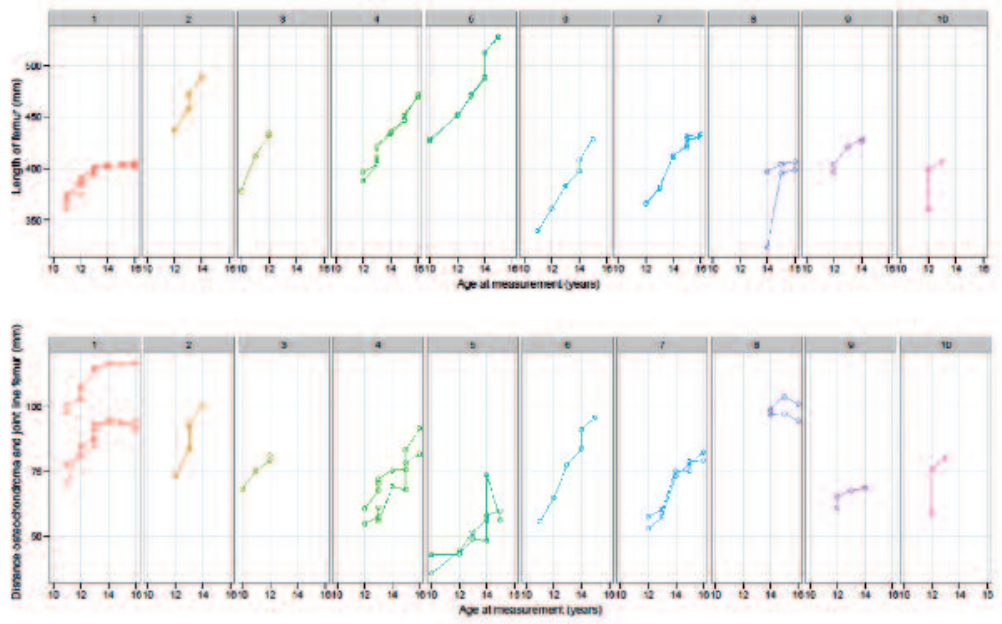

C
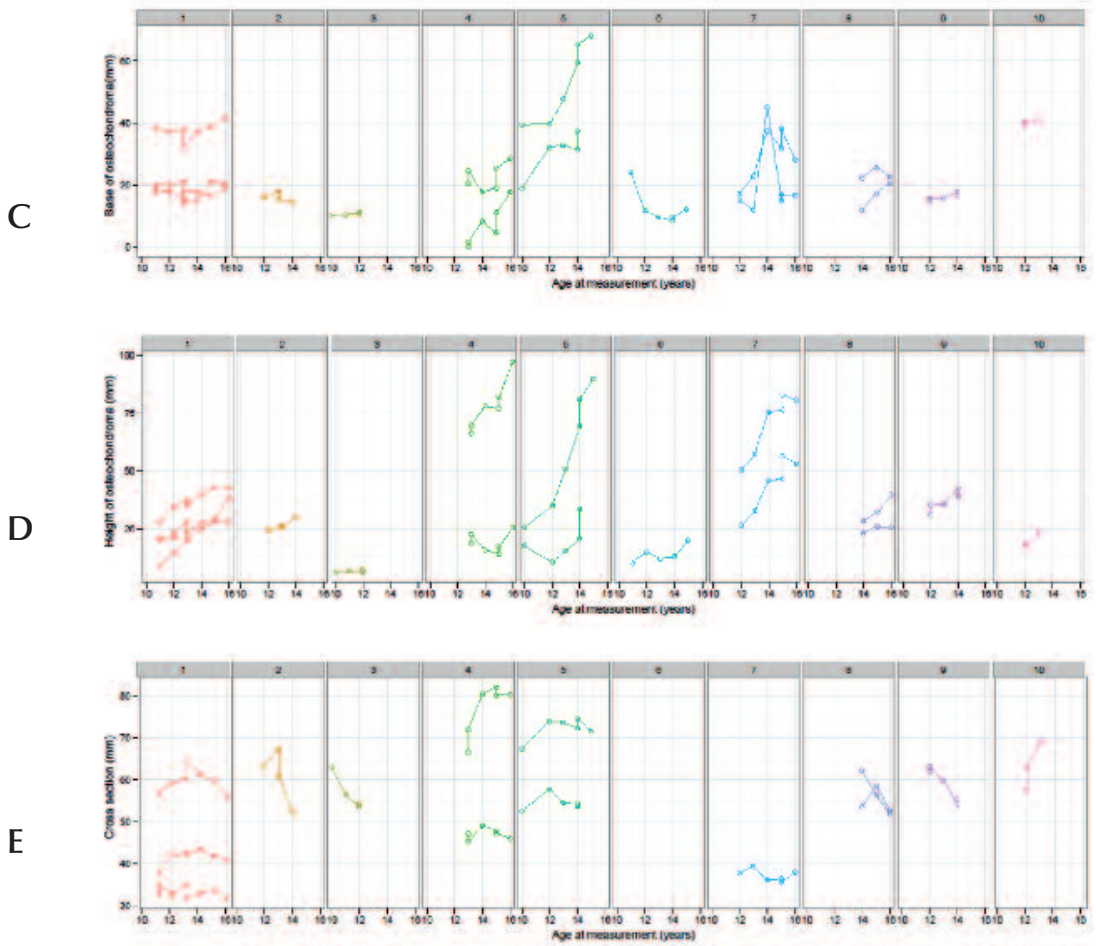

Figure 3: Mean of measurements of both observers in time for each patient (1-10). Multiple lines within a patient represent multiple osteochondromas. 


\section{Chapter 3}

Both the total length of the femur and the height of the osteochondroma could be reliably measured. The length of the femur and the height of the osteochondroma increased over time during growth in all patients (Figures 3A-E). The femoral growth is shown in table 1 (Table 1). The length of the femur increased over time with a varying rate per patient from $3 \mathrm{~mm}$ up to $3 \mathrm{~cm}$ per year (average $1,73 \mathrm{~cm} /$ year, std. $0,84)$. The height of the osteochondromas varied widely. The increase in height varied from no growth (or even decrease of height) to $33,1 \mathrm{~mm}$.

No newly formed osteochondromas were detected proximal to the existing osteochondromas of the distal femur, indicating that all newly formed osteochondromas were formed closer to the epiphyseal line than the already existing ones.

\begin{tabular}{lccc} 
No & Length femur (A) first $(\mathrm{mm})$ & Length femur $(\mathrm{A})$ last $(\mathrm{mm})$ & Increased Length femur (cm/year) \\
\hline 1 & 362,1 & 405,8 & 0,89 \\
2 & 369,5 & 402,3 & 0,67 \\
3 & 369,5 & 402.3 & 0,67 \\
4 & 436,7 & 490,0 & 2,4 \\
5 & 378,8 & 437,1 & 3,0 \\
6 & 392,4 & 470,9 & 1,8 \\
7 & 392,4 & 470,9 & 1,8 \\
8 & 428,1 & 527,5 & 2,1 \\
9 & 428,1 & 527,5 & 2,1 \\
10 & 333,9 & 432,3 & 2,2 \\
11 & 366,5 & 431,7 & 1,4 \\
12 & 366,5 & 431,7 & 1,4 \\
13 & 322,7 & 397,4 & 2,7 \\
14 & 398,9 & 407,4 & 0,3 \\
15 & 388,6 & 416,5 & 1,3 \\
16 & 350,8 & 408,8 & 3,0 \\
\hline
\end{tabular}

Table 1: Mean results both first and last measurment of the length of the femur and the average growth per year. 


\section{Discussion}

The aims of this study were to asses whether it was possible to accurately determine growth of osteochondromas on plain X-rays, to assess which parameters best describe the relationship between growth of the femur and development of osteochondromas, to assess whether the osteochondromas' place of origin and its positional relation to the femur changes during skeletal growth. The results indicate that the length of the femur and the height of the osteochondromas could be reliably measured on plain X-rays with high inter-rater agreement. Both increase over time during growth. However, measurements of the distance of the osteochondroma to the joint line of the knee, femoral cross section and the base of the osteochondroma resulted in low reliability. Therefore we are unable to provide an answer to the first question, which osteochondroma growth parameters best describe the relationship between growth of the femur and development and location of osteochondromas.

The distal femoral growth plate is responsible for $1,4 \mathrm{~cm}$ of lengthening of the femur per year in normal femora, as shown by Sissons and Kember ${ }^{25}$. Comparing this normal lengthening of a distal femur to the lengthening of the femur in $\mathrm{HMO}$ affected children, it seems that the HMO femora have a slightly faster growing rate (average in this study $1,73 \mathrm{~cm} /$ year). Several studies show that patients with multiple $\mathrm{HMO}$ have shortened long bones ${ }^{4,26,27}$. The shortening was proposed to be the result of steal of the longitudinal growth into osteochondromas. Jones et al. ${ }^{27}$ induced osteochondromagenesis at different time points during skeletal growth in a mouse genetic model and described that these mice with osteochondromas presented with shorter femora and tibiae than controls. They also concluded that the shortening did not correlate with osteochondroma volumetric growth, suggesting that even though a steal phenomenon seems apparent, some other unknown mechanism must be contributing to the short bone phenotype.

If steal influenced the growth, one would expecte a slower growth rate depending on the distance of the osteochondroma to the epiphyseal line. Unfortunately, the joint line parameters in this study could not be reliably measured; therefore it does not support the hypothesis of a steal phenomenon. Nor does the slightly increased growing rate support this theory. 


\section{Chapter 3}

No newly formed osteochondromas proximal to the existing osteochondroma in the distal femur were observed during longitudinal growth. This confirms the commonly accepted hypothesis that osteochondroma are formed in or near the epiphyses ${ }^{4,26,27}$. Based on the hypothesis that the distal femur grows distal while the osteochondroma remains in its place of origin, we expected that the distance of the osteochondroma to the joint line of the knee would increase over time during lengthening of the femur. Similarly, we anticipated on the cross section of the femur to decrease due to an increasingly more diaphyseal location of the osteochondroma as the femur lengthens. We cannot verify or reject this hypothesis.

Because the distance of the osteochondroma to the joint line of the knee increased during growth and the new osteochondromas were only formed closer to the epiphysis, it increases the likelihood that the osteochondromas are formed at or near the epiphyses during growth and stay in their place of origin, as the femur grows more distal, resulting in a more proximal position of the osteochondromas over time.

Some potential limitations of this study should be discussed. First, the studied cohort was small. Second, our study only assessed plain X-rays which were taken according to the local hospital protocol, possibly resulting in small differences in the rotation of the legs that might have influenced the measurements. These influences were noted in the data by for instance a decrease in height of the osteochondroma at a certain time point followed by an increase again in the next time point, however were corrected for in the analyses. Should a prospective radiological follow-up study start, we then advocate a more standardized radiographic procedure, for instance with the use of Roentgen stereophotogrammetric analysis (RSA) with fixed marker points in the diaphysis and in the osteochondromas.

Despite the aforementioned limitations, this study is the first radiological analysis of longitudinal osteochondroma changes and it showed that parameters length of the femur and height of the osteochondroma could be reliably measured on plain X-rays. However, other measurements such as femoral cross section, the base of the osteochondroma and the distance of the osteochondroma to the joint line of the knee resulted in low reliability. 


\section{Conclusions}

Length of the femur and height of the osteochondroma can be reliably measured on plain X-rays and both increased over time during growth. No newly formed osteochondromas proximal to the existing osteochondroma were observed during longitudinal growth in this study. It seems likely that the osteochondroma are formed at or near the epiphyses during growth and stay in their place of origin, as the femur grows more distal, realizing a more proximal position of the osteochondroma over time.

\section{Acknowledgements}

We thank Areej Alsadah, Maastricht University, for collecting clinical and X-ray data. 


\section{Chapter 3}

\section{REFERENCES}

1. Bovee JV. Multiple osteochondromas. Orphanet J Rare Dis 2008;3: 3.

2. Kitsoulis P, Galani V, Stefanaki K, Paraskevas G, Karatzias G, Agnantis NJ, Bai M. Osteochondromas: review of the clinical, radiological and pathological features. In Vivo 2008;22: 633-46.

3. Tompkins M, Eberson C, Ehrlich M. Hemiepiphyseal stapling for ankle valgus in multiple hereditary exostoses. Am J Orthop (Belle Mead NJ) 2012;41: E23-6.

4. Clement ND, Duckworth AD, Baker AD, Porter DE. Skeletal growth patterns in hereditary multiple exostoses: a natural history. J Pediatr Orthop B 2012;21: 150-4.

5. Khurana J, F. FA-K, Bovee J. Osteochondroma. World Health Organisation Classification of tumours. Pathology and Genetics of Tumours of Soft Tissue and Bone 2002: 234-236.

6. Bovee J, Hogendoorn PC. Multiple Osteochondromas. World Health Organisation Classification of tumours. Pathology and Genetics of Tumours of Soft Tissue and Bone 2002: 260-362.

7. Solomon L. Hereditary Multiple Exostosis. Am J Hum Genet 1964;16: 351-63.

8. Guo XL, Deng Y, Liu HG. Clinical characteristics of hereditary multiple exostoses: a retrospective study of mainland chinese cases in recent 23 years. J Huazhong Univ Sci Technolog Med Sci 2014;34: 42-50.

9. Greenspan A. Tumors of cartilage origin. Orthop Clin North Am 1989;20: 347-66.

10. Porter DE, Lonie L, Fraser M, Dobson-Stone C, Porter JR, Monaco AP, Simpson AH. Severity of disease and risk of malignant change in hereditary multiple exostoses. A genotypephenotype study. J Bone Joint Surg Br 2004;86: 1041-6.

11. Porter DE, Simpson AH. The neoplastic pathogenesis of solitary and multiple osteochondromas. J Pathol 1999;188: 119-25.

12. Stieber JR, Dormans JP. Manifestations of hereditary multiple exostoses. J Am Acad Orthop Surg 2005;13: 110-20.

13. Shapiro F, Simon S, Glimcher MJ. Hereditary multiple exostoses. Anthropometric, roentgenographic, and clinical aspects. J Bone Joint Surg Am 1979;61: 815-24.

14. Clement ND, Porter DE. Can deformity of the knee and longitudinal growth of the leg be predicted in patients with hereditary multiple exostoses? A cross-sectional study. Knee 2014;21: 299-303. 
15. Schmale GA, Conrad EU, 3rd, Raskind WH. The natural history of hereditary multiple exostoses. J Bone Joint Surg Am 1994;76: 986-92.

16. Fairbank T. An Atlas of general affectations of the skeleton. 1951: 60-61.

17. $\mathrm{D}^{\prime}$ Ambrosia R, Ferguson $A B$, Jr. The formation of osteochondroma by epiphyseal cartilage transplantation. Clin Orthop Relat Res 1968;61: 103-15.

18. Milgram JW. The origins of osteochondromas and enchondromas. A histopathologic study. Clin Orthop Relat Res 1983: 264-84.

19. Mansoor A, Beals RK. Multiple exostosis: a short study of abnormalities near the growth plate. J Pediatr Orthop B 2007;16: 363-5.

20. Chen CC, Barnhart HX. Assessing agreement with intraclass correlation coefficient and concordance correlation coefficient for data with repeated measures. Computational Statistics \& Data Analysis 2013;60: 132-145.

21. Carrasco JL, Martinez JP, L J. CCC-RM. R package version 1.1. In; 2012.

22. Carrasco JL, Phillips BR, Puig-Martinez J, King TS, Chinchilli VM. Estimation of the concordance correlation coefficient for repeated measures using SAS and R. Computer Methods and Programs in Biomedicine 2013;109: 293-304.

23. Carstensen B, Gurrin L, Ekstrom C, Figurski M. MethComp: Functions for analysis of agreement in method comparison studies. R package version 1.22. In; 2013.

24. team RC. A language and evironment for statistical computing. R Foundation for Statistical Computing, Vienna, Austria. In; 2014.

25. Sissons HA, Kember NF. Longitudinal bone growth of the human femur. Postgrad Med J 1977;53: 433-7.

26. Porter DE, Emerton ME, Villanueva-Lopez F, Simpson AH. Clinical and radiographic analysis of osteochondromas and growth disturbance in hereditary multiple exostoses. J Pediatr Orthop 2000;20: 246-50.

27. Jones KB, Datar M, Ravichandran S, Jin H, Jurrus E, Whitaker R, Capecchi MR. Toward an understanding of the short bone phenotype associated with multiple osteochondromas. J Orthop Res 2013;31: 651-7. 


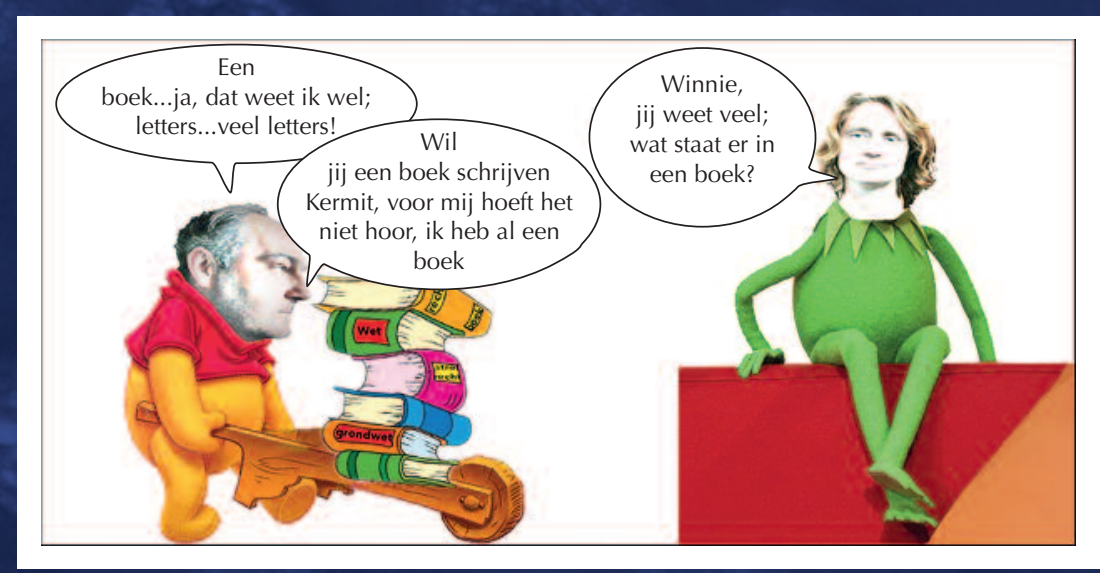




\section{CHAPTER FOUR}

\section{Skeletal maturity of children with Hereditary Multiple}

Osteochondromas: is diminished stature due to a systemic influence?

Heleen M. Staal, Annemarie L. Goud, Henk-Jan van der Woude, M. Adhiambo Witlox, S. John Ham, Simon GF. Robben, Marjolein HG. Dremmen, Lodewijk W. van Rhijn.

Adapted from

J Child Orthop. 2015 Oct; 9(5):397-402. doi: 10.1007/s11832-015-0680-x. Epub 2015 Sep 1. 


\section{Chapter 4}

\section{AbStRACT}

Background: Hereditary Multiple Osteochondromas (HMO) is an autosomal dominant inherited disease caused by mutated exostosin genes. It mostly affects the long bones and can lead to growth disturbances, especially disproportionally short stature. Both the local effect on growth plates and the systemic influence of the gene disorder on growth mechanisms might explain the diminished stature.

Purpose: The hypothesis of this study is that the diminished stature in adults with $\mathrm{HMO}$ is due to a systemic influence leading to early skeletal maturation and early closure of the growth plate. Therefore in these patients the skeletal age in adolescence is hypothesized to be higher than the calendar age.

Methods: Of $50 \mathrm{HMO}$ affected children, radiographs of the left hand were collected. The skeletal age was calculated using these radiographs according to the Greulich-Pyle bone scale and was compared to the calendar age at the time of radiography.

Results: Children aged 3-12 years had a significantly lower skeletal age compared to the calendar age $(p=0.030)$. Children aged 12-17 years had a significantly higher skeletal age $(p=0,019)$, especially boys. Skeletal maturation in children with $\mathrm{HMO}$ therefore differs from their peers.

Conclusion: In this study the skeletal age in younger children with Hereditary Multiple Osteochondromas is lower than their calendar age. For adolescents, particularly boys, this is reversed, suggesting an earlier or faster closure of the growth plates. These findings support a systemic influence of the gene defect on growth rate. 


\section{INTRODUCTION}

Hereditary Multiple Osteochondromas (HMO), sometimes also referred to as Hereditary Multiple Exostoses (HME) is characterized by the outward growth of cartilage-capped bone tumours called osteochondromas. It is an autosomal dominant inherited disorder caused by mutated exostosin genes (EXT1 or EXT2) ${ }^{1}$. The osteochondromas develop in the first decade of life and cease to grow once the patient reaches skeletal maturity. The long bones are almost always affected, but osteochondromas are also found on the scapula, the ribs and the pelvis. HMO can lead to growth disturbances including unequal limb length, joint deformity and disproportionally short stature ${ }^{2-6}$. A considerable number of patients suffer from pain or discomfort due to the disorder, significantly affecting quality of life ${ }^{7}$. Diminished or short stature is a common feature in patients with $\mathrm{HMO}$. In the majority of the patients their height is below normal average height, but within a normal range ${ }^{7-9}$. The origin of this short or diminished stature in HMO patients is still unknown. Hypothetically it might be due to a local effect from osteochondromas on the local growth plates or it might be due to a systemic effect.

This local effect of osteochondromas on the growth plate is generally known to lead to leg length discrepancy, Madelungs deformity and other growth disturbances. An analysis performed by Porter et al. in 2000 showed an inverse correlation between osteochondroma size and relative bone length, suggesting that the growth retardation in $\mathrm{HMO}$ might result from the local effects of enlarging osteochondromas rather than a systemic effect caused by skeletal dysplasia ${ }^{10}$. Several studies have described the systemic influence of the gene defect and its relation to the growth plate as a possible cause of the short or diminished stature. Jones et al. ${ }^{9}$ demonstrated in a mouse genetic model that mice with osteochondromas had shorter femora and tibiae than controls. The volume of the osteochondromas, however, did not correlate with longitudinal shortening. In their model loss of heterozygosity for EXT1 was sufficient to drive bone shortening. Other mouse models of $\mathrm{HMO}$ have provided more information about the pathogenesis of osteochondromas and the role of $\mathrm{HS}$ in normal growth plate development ${ }^{11-13}$.

Both the local effect of osteochondromas and the systemic HS influencing effect of the EXT genes may explain the diminished stature observed in patients with $\mathrm{HMO}$. 


\section{Chapter 4}

In addition, we know that $\mathrm{HMO}$ affected adults have a shorter stature than expected. However, a study performed in 2012 by Clement et al. showed that this diminished stature is relative; in the adolescent age group the stature of $\mathrm{HMO}$ children seemed to be taller than that of their peers without the disorder ${ }^{14}$. Hypothetically, the discrepancy between this relatively tall stature during adolescence and a diminished stature in adulthood could be due to a systemic influence affecting the maturation of the epiphyses. This in turn could lead to early puberty and early closure of the growth plate. The EXT gene disorder affects the HS synthesis, which could have a systemic effect and influence on the moment of growth plate closure.

Because skeletal maturation is controlled by hormones and the same hormones also govern the timing of puberty and might be influenced by the systemic gene defect in the EXT genes, the hypothesis of this study is that the relatively tall stature during adolescence and the diminished stature in adults with $\mathrm{HMO}$ is due to a systemic influence leading to early maturation of the epiphysis and early closure of the growth plate. If so, the skeletal age in adolescence should be higher than the related calendar age.

The aim of this study is to test the hypothesis that the epiphyses closes early in $\mathrm{HMO}$ affected children.

\section{MATERIALS AND METHODS}

Data were collected from the joint $\mathrm{HMO}$ database of the two Dutch national tertiary referral centres for $\mathrm{HMO}$, namely OLVG in Amsterdam and Maastricht University Medical Centre. From this database all 50 patients under the age of 20 were selected, of whom a radiograph of the left hand had been made for clinical indications between 1995 and March 2015. To determine the skeletal age the Greulich and Pyle method was used, which has been elaborately described previously ${ }^{15}$. In short, this method uses a standardised set of x-rays of the left hand and wrist, against which the image of the subject is compared.

In all 50 cases two independent radiologists, both highly experienced in skeletal maturity assessment, evaluated the X-rays. Both were blinded to the original report and the calendar age. When the two independent readings were concordant 
(ie less than 6 months difference) the average was used. In case of discordance, images were re-evaluated by the two readers combined, until consensus was reached. The calendar age and skeletal age were recorded in years and months. For statistical analyses, paired T-test was used.

\section{Results}

There were 23 males and 27 females. Skeletal age could be measured in all patients, despite the presence of osteochondromas in all patients and severe deformation of the wrist in 11. The epiphyseal lines were assessable in all radiographs. The patients calendar age varied from 3 years and 9 months to 19 years and 3 months. There were 11 patients aged 3-8 years at the time of radiography, 16 patients aged 8-12 years, 18 patients aged 12-17 years, and five patients 17-20 years. The average calendar age was 11 years and 6 months (SD, 4.2 years). All results are shown in table 1.

In the $\mathrm{HMO}$ affected children under 12 years of age the average skeletal age was less than their calendar age in both boys and girls. In this group the average skeletal age was 7.39 ; the average calendar age was $7.79(p=0.030)$. In the group aged 12-14 (years) there seemed to be no difference between the $\mathrm{HMO}$ affected children and their unaffected peers. After the age of 14 the skeletal age overtook the calendar age. In boys, this was more pronounced than in girls (figures 1 and 2).

Adolescents between 12 and 17 years old had a significant older skeletal age compared to their peers $(p=0.019)$. The average calendar age was 13.95 and the average skeletal age was 14.37. As can be seen from figure 1 for boys and figure 2 for girls, the difference was mainly caused by the skeletal age of the boys in this group. The boys between 12 and 17 had a significantly older skeletal age with a $p$ value of 0.011 .

Above the age of 17 there was no statistical difference. 


\section{Chapter 4}

\begin{tabular}{|c|c|c|c|c|c|}
\hline Patient no. & $\begin{array}{l}\text { Calendar age } \\
\text { (years) }\end{array}$ & $\begin{array}{l}\text { Skeletal age } \\
\text { (years) }\end{array}$ & Patient no. & $\begin{array}{l}\text { Calendar age } \\
\text { (years) }\end{array}$ & $\begin{array}{l}\text { Skeletal age } \\
\text { (years) }\end{array}$ \\
\hline 1 & 3,75 & 3,5 & 26 & 11,58 & 12 \\
\hline 2 & 4,5 & 3,5 & 27 & 11,92 & 12 \\
\hline 3 & 4,75 & 3,5 & 28 & 12 & 12 \\
\hline 4 & 5,25 & 5 & 29 & 12 & 13 \\
\hline 5 & 5,58 & 5 & 30 & 12,67 & 12 \\
\hline 6 & 5,83 & 5,83 & 31 & 13,5 & 14,75 \\
\hline 7 & 5,83 & 5 & 32 & 13,75 & 14 \\
\hline 8 & 5,92 & 5,75 & 33 & 13,83 & 14,5 \\
\hline 9 & 6,42 & 6,83 & 34 & 14 & 15 \\
\hline 10 & 6,5 & 8 & 35 & 14,08 & 14 \\
\hline 11 & 7 & 6,83 & 36 & 14,67 & 15,5 \\
\hline 12 & 8 & 6 & 37 & 14,75 & 17 \\
\hline 13 & 8,25 & 7,83 & 38 & 15,33 & 16 \\
\hline 14 & 8,67 & 7,83 & 39 & 15,33 & 15,5 \\
\hline 15 & 8,67 & 8 & 40 & 15,42 & 15,5 \\
\hline 16 & 8,92 & 7,83 & 41 & 15,5 & 14,75 \\
\hline 17 & 9,67 & 8,83 & 42 & 15,5 & 15 \\
\hline 18 & 9,75 & 10 & 43 & 15,67 & 16 \\
\hline 19 & 10 & 8,83 & 44 & 16 & 16 \\
\hline 20 & 10,08 & 10 & 45 & 17 & 16 \\
\hline 21 & 10,08 & 11 & 46 & 17,08 & 17 \\
\hline 22 & 11,08 & 9 & 47 & 18 & 18 \\
\hline 23 & 11,17 & 12 & 48 & 18,08 & 18 \\
\hline 24 & 11,42 & 11,5 & 49 & 18,75 & 19 \\
\hline 25 & 11,58 & 13 & 50 & 19,25 & 19 \\
\hline
\end{tabular}

Table 1: Results of all $50 \mathrm{HMO}$ affected patients, calendar versus skeletal age. 


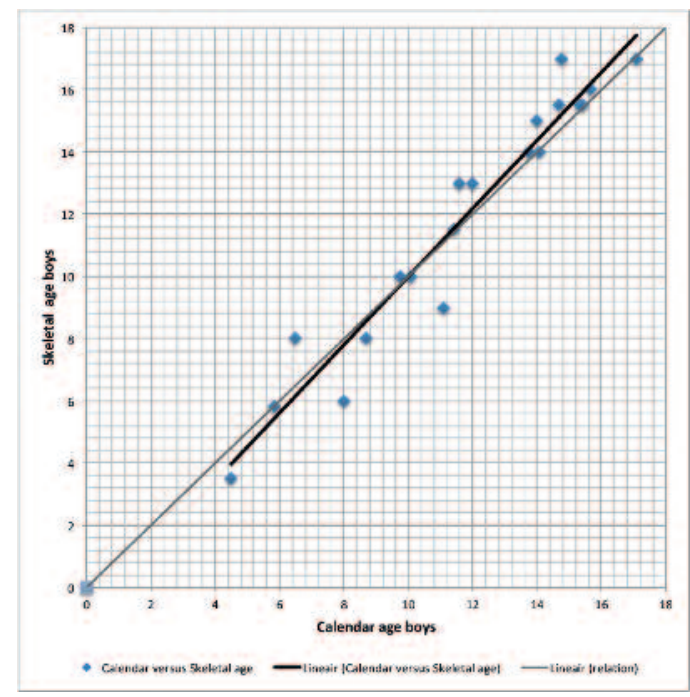

Figure 1: The Calender versus Skelatal age, results for boys (under 17) plotted (blue diamonds) and their average (black line). The grey line represents the normal line.

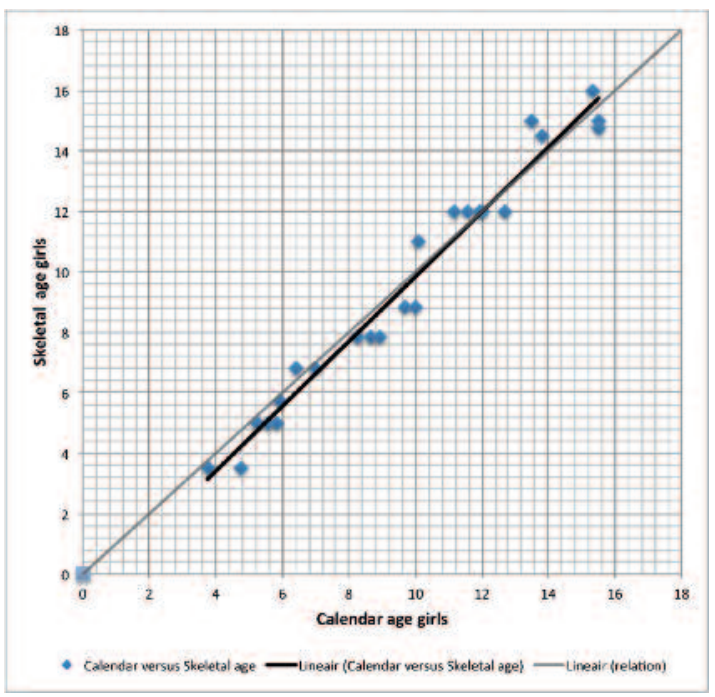

Figure 2: The Calender versus Skelatal age, results of the girls (under 17) plotted (blue diamonds) and their average (black line). The grey line represents the normal line. 


\section{Chapter 4}

\section{Discussion}

The present study shows that the skeletal age and calendar age in $\mathrm{HMO}$ children is different from their peers. In the younger children the skeletal age seems lower and in adolescents it seems higher. The turning point lies around the age of 12 at the start of adolescence. Possibly, the growth plates in patients with $\mathrm{HMO}$ close earlier or faster, particularly in boys.

Our results support the results of a study performed in 2012 by Clement et al. Their study showed that the final diminished stature of $\mathrm{HMO}$ patients was relative. They reported that in the adolescent age group the stature of $\mathrm{HMO}$ children was taller than that of their peers without the disorder, after the age of 15 they were shorter than their peers. Especially the leg length was reduced ${ }^{14}$. The younger skeletal age in the pre-adolescent children can explain their relatively taller stature. A younger skeletal age is assessed when the growth plates are wider. This wideness of the growth plates can be caused by the systemic effect of the EXT genes.

Several studies have described this systemic influence of the gene defect. Jones et al. ${ }^{9}$ concluded that chondrocytes lacking functional heparan sulphate (HS) influence physeal signalling in general, rather than stealing growth potential focally. Koziel et al. ${ }^{11}$ described a genetic mouse line expressing a truncated form of EXT1 that displayed shortened skeletal elements and fused vertebrae. EXT genes belong to a family of glycosyltransferases necessary for the synthesis of the heparan sulphate. $\mathrm{HS}$ regulates signalling of several growth factors. Reduced HS synthesis results in an elevation of Indian hedgehog (lhh), a protein involved in chondrocyte differentiation. Misexpression of Ihh causes, amongst others, changes in the expression of prochondrogenic Bone Morphogenetic Proteins (BMPs), altering the differentiation of cells in the growth plate as well as the bordering perichondrium ${ }^{12}$. Members of both the BMP and fibroblast growth factor (FGF) families are expressed in growth plate and/or perichondrium and are a part of interactive loops regulating thh and Parathyroid Hormone related Protein (PTHrP) expression and the overall growth plate activities $^{12,13}$. HS is needed to restrain pro-chondrogenic signalling proteins and restricts the chondrogenesis. If the HS levels are low, this process is disturbed and could lead to increased chondrogenic activity including the activity of cartilage cells in the growth plate. This might lead to the wider growth plates. Because HS also 
influences the Ihh activity and Ihh seems to orchestrate the chondrocyte proliferation this might be a second cause for the wider growth plates in the preadolescent $\mathrm{HMO}$ children and thus the relatively young skeletal age ${ }^{16,17}$.

The systemic effect of the genetic disorder can also explain the older skeletal age in the adolescent children due to their growth plates closing earlier. Ihh is essential for normal chondrocyte maturation, regulating both proliferation and differentiation. Ihh also regulates proliferation of chondrocytes by directly controlling the rate of cell division of columnar/proliferative chondrocytes ${ }^{18,19}$. Inactivation of Ihh in chondrocytes leads to abrupt fusion of the epiphyseal growth plate in mice $e^{20,21}$. In humans an inactivating mutation in Ihh results in acrocapitofemoral dysplasia, which is associated with premature closure of the growth plates ${ }^{22-26}$.

Several studies have shown that the stature was more severely affected in patients with an EXT1 mutation ${ }^{14,27,28}$. In this study we did not sub-select the different gene defect genotypes and therefore cannot contribute to this discussion.

Both mice and human studies showed that the relatively short stature was disproportionate. The sitting height was less affected than leg length, indicating more involvement of the limbs than of the axial skeleton ${ }^{29,30}$. This phenomenon can also be explained by the early closure of the growth plate of the distal femur. The femoral contribution to length is not an even contribution during growth. Approximately $70 \%$ of growth in the femur occurs at the distal growth plate but the proportion of growth occurring in the distal femoral growth plate varies with age, from $55 \%-60 \%$ around age 7 to $90 \%$ at age from 14 to $16^{31}$. This explains why early closure of the growth plates can lead to relatively short femora and therefore legs, as particularly femoral growth potential is lost.

All of the above seems to support the systemic skeletal dysplasia theory rather than a local origin of the disproportional growth, even though it contradicts the findings described by Porter et al. Their study described a clinical and radiographic analysis of paired bone length in a $\mathrm{HMO}$ cohort. They found that the local presence of osteochondromas was associated with growth disturbance. There was an inverse correlation between osteochondroma size and relative bone length. Their conclusion was that the growth retardation might result from a local effect ${ }^{10}$. Of note, half of their study patients were adults, and this might have influenced their findings. The study was performed using radiographs of the forearm comparing the length of the two forearm 


\section{Chapter 4}

bones. Since the epiphyses in these bones do not grow at the same rate, the growth might be influenced at a different pace. Also the position of the osteochondromas might play an important role in the forearm, since the interosseous membrane connects the two bones and the relative position of the osteochondroma in relation to this membrane might play a role in the growth disturbance. The forearm bones cannot be measured as two separate bones because of their close relation. Furthermore, the finding of the inverse correlation could be due to the severity of the disease rather than the actual size of osteochondromas. More and bigger osteochondromas means more severe $\mathrm{HMO}$ and thus more influence of the gene dysfunction on the biochemical setup. A mouse study by Jones does not support the relation between the volume of the osteochondromas and the growth plate influence either ${ }^{32}$.

A clear limitation to the present study is the inter-individual variation of the skeletal age. Different population groups mature at different speeds. For instance, healthy living children mature faster than children living under poor conditions. Besides that, the standard deviation of skeletal age at a given age can vary approximately 1 year. The number of patients in this study is relatively low to compensate for this large deviation. For future studies we advocate to select more patients and to divide them by their genotype. In this way the underlying gene defect and its relation to the growth disturbance can be assorted.

This study has shown that in $\mathrm{HMO}$ patients the skeletal age differs from their calendar age. This is of direct clinical relevance in the planning of epiphysiodesis in leg length discrepancy and hemi-epiphysiodesis in axial deformities in HMO patients and especially in boys. Individual longitudinal follow-up of bone growth is advised.

\section{Conclusion}

The skeletal age in younger children with Hereditary Multiple Osteochondromas is lower than their calendar age, while for adolescent boys it is higher. The turning point lies around the start of adolescence. This phenomenon may explain diminished stature in $\mathrm{HMO}$. These findings support a systemic influence of the gene defect on growth rate. 


\section{REFERENCES}

1. Wuyts, W., et al., Hereditary Multiple Osteochondromas., in A.M. Pagon RA, Ardinger HH, et al, Editor. 2000 University of Washington Seattle. p. 220-7.

2. Khurana, J., F.A.-K. F., and J. Bovee, Osteochondroma. World Health Organisation Classification of tumours. Pathology and Genetics of Tumours of Soft Tissue and Bone 2002: p. 234-236.

3. Bovee, J. and P.C. Hogendoorn, Multiple Osteochondromas. World Health Organisation Classification of tumours. Pathology and Genetics of Tumours of Soft Tissue and Bone, 2002: p. 260-362.

4. Hennekam, R.C., Hereditary multiple exostoses. J Med Genet, 1991. 28(4): p. 262-6.

5. Schmale, G.A., E.U. Conrad, 3rd, and W.H. Raskind, The natural history of hereditary multiple exostoses. J Bone Joint Surg Am, 1994. 76(7): p. 986-92.

6. Wicklund, C.L., et al., Natural history study of hereditary multiple exostoses. Am J Med Genet, 1995. 55(1): p. 43-6.

7. Goud, A.L., et al., Pain, physical and social functioning, and quality of life in individuals with multiple hereditary exostoses in The Netherlands: a national cohort study. J Bone Joint Surg Am, 2012. 94(11): p. 1013-20.

8. Solomon, L., Bone growth in diaphysial aclasis. J Bone Joint Surg Br, 1961. 43-B: p. 70016.

9. Stieber, B.A., K.A. Pierz, and J.P. Dormans, HME: a current understanding of clinical and genetic advances. . Univ Penn Orthop J., 2001. 114: p. 39-48

10. Porter, D.E., et al., Clinical and radiographic analysis of osteochondromas and growth disturbance in hereditary multiple exostoses. J Pediatr Orthop, 2000. 20(2): p. 246-50.

11. Koziel, L., et al., Ext1-dependent heparan sulfate regulates the range of Ihh signaling during endochondral ossification. Dev Cell, 2004. 6(6): p. 801-13.

12. Pathi, S., et al., Interaction of Ihh and BMP/Noggin signaling during cartilage differentiation. Dev Biol, 1999. 209(2): p. 239-53.

13. Zou, H., et al., Distinct roles of type I bone morphogenetic protein receptors in the formation and differentiation of cartilage. Genes Dev, 1997. 11(17): p. 2191-203.

14. Clement, N.D., et al., Skeletal growth patterns in hereditary multiple exostoses: a natural history. J Pediatr Orthop B, 2012. 21(2): p. 150-4. 


\section{Chapter 4}

15. Greulich, W.W. and P. S.I., Radiographic Atlas of Skeletal Development of the Hand and Wrist. 1959 Stanford University Press.

16. Lanske, B., et al., PTH/PTHrP receptor in early development and Indian hedgehogregulated bone growth. Science, 1996. 273(5275): p. 663-6.

17. Enomoto-Iwamoto, M., et al., Hedgehog proteins stimulate chondrogenic cell differentiation and cartilage formation. J Bone Miner Res, 2000. 15(9): p. 1659-68.

18. Long, F., et al., Genetic manipulation of hedgehog signaling in the endochondral skeleton reveals a direct role in the regulation of chondrocyte proliferation. Development, 2001. 128(24): p. 5099-108.

19. Kobayashi, T., et al., Indian hedgehog stimulates periarticular chondrocyte differentiation to regulate growth plate length independently of PTHrP. J Clin Invest, 2005. 115(7): p. 173442.

20. Maeda, Y., et al., Indian Hedgehog produced by postnatal chondrocytes is essential for maintaining a growth plate and trabecular bone. Proc Natl Acad Sci U S A, 2007. 104(15): p. 6382-7.

21. Hirai, T., et al., Parathyroid hormone/parathyroid hormone-related protein receptor signaling is required for maintenance of the growth plate in postnatal life. Proc Natl Acad Sci U S A, 2011. 108(1): p. 191-6.

22. Hellemans, J., et al., Homozygous mutations in IHH cause acrocapitofemoral dysplasia, an autosomal recessive disorder with cone-shaped epiphyses in hands and hips. Am J Hum Genet, 2003. 72(4): p. 1040-6.

23. St-Jacques, B., M. Hammerschmidt, and A.P. McMahon, Indian hedgehog signaling regulates proliferation and differentiation of chondrocytes and is essential for bone formation. Genes Dev, 1999. 13(16): p. 2072-86.

24. Cortes, M., A.T. Baria, and N.B. Schwartz, Sulfation of chondroitin sulfate proteoglycans is necessary for proper Indian hedgehog signaling in the developing growth plate. Development, 2009. 136(10): p. 1697-706.

25. Huegel, J., et al., Perichondrium phenotype and border function are regulated by Ext1 and heparan sulfate in developing long bones: a mechanism likely deranged in Hereditary Multiple Exostoses. Dev Biol, 2013. 377(1): p. 100-12.

26. Staal, H.M., et al., Current Knowledge on Exostoses Formation in Hereditary Multiple Exostoses: Where do Exostoses Originate and in What Way is their Growth Regulated. Hereditary Genetics 2014, 3:3 
27. Pedrini, E., et al., Genotype-phenotype correlation study in 529 patients with multiple hereditary exostoses: identification of "protective" and "risk" factors. J Bone Joint Surg Am, 2011. 93(24): p. 2294-302.

28. Porter, D.E., et al., Severity of disease and risk of malignant change in hereditary multiple exostoses. A genotype-phenotype study. J Bone Joint Surg Br, 2004. 86(7): p. 1041-6.

29. Shapiro, F., S. Simon, and M.J. Glimcher, Hereditary multiple exostoses. Anthropometric, roentgenographic, and clinical aspects. J Bone Joint Surg Am, 1979. 61(6A): p. 815-24.

30. Sgariglia, F., et al., Epiphyseal abnormalities, trabecular bone loss and articular chondrocyte hypertrophy develop in the long bones of postnatal Ext1-deficient mice. Bone, 2013. 57(1): p. 220-31.

31. Pritchett, J.W., Longitudinal growth and growth-plate activity in the lower extremity. Clin Orthop Relat Res, 1992(275): p. 274-9.

32. Jones, K.B., et al., Toward an understanding of the short bone phenotype associated with multiple osteochondromas. J Orthop Res, 2013. 31(4): p. 651-7. 

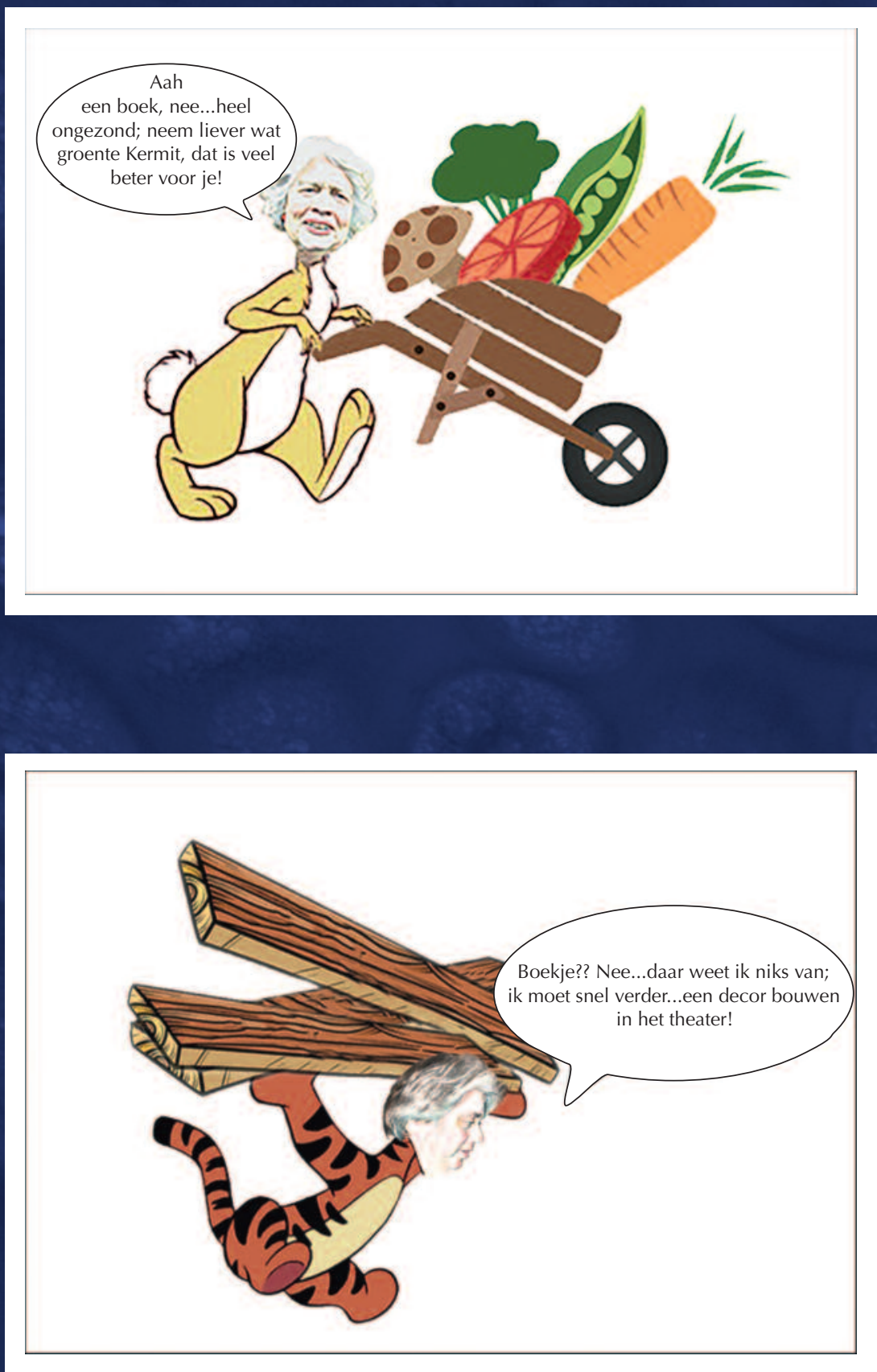


\section{Chapter five}

\section{The microarchitecture of bone in osteochondromas, a pilot study with Micro Computed Tomography Imaging}

Heleen M. Staal, Wouter Nijhuis, M. Adhiambo Witlox, Koen RJ. Boom, Giovanni Paffen, S. John Ham, Tim JM. Welting, Bert van Rietbergen, Lodewijk W. van Rhijn. 


\section{Chapter 5}

\section{Abstract}

Osteochondromas are the most common benign bone tumours. They mostly develop in the metaphysis of long bones. Osteochondromas develop in an off-axis position and are expected to carry little mechanical loading. On clinical removal the osteochondromas appear to have a less dense, osteoporotic like, structure. Their bone architecture is studied in nine resected osteochondromas using micro-CT. The trabecular morphology is quantified and findings are compared to histological findings and to values from literature on normal bones in the same age groups. The micro-CT shows that osteochondromas have a normal trabecular number and bone volume fraction, but the trabeculae are wider spaced and thicker than in regular trabecular bone.

This study is the first to show that, compared to that of age matched controls, the microarchitecture of the osteochondromas show less but thicker trabeculae that are wider separated with a normal bone volume fraction. 


\section{INTRODUCTION}

Osteochondromas are defined as a cartilage-capped bony outgrowth on the external surface of bones consisting of a marrow cavity that is continuous with that of underlying bone $^{1,2}$. They most often occur at the metaphysisis of long bones. Osteochondromas can be single or multiple. Patients suffering from Hereditary Multiple Osteochondromas $(\mathrm{HMO})$ are known to have many osteochondromas. If the osteochondromas result in pressure on the overlying tissues, patients can suffer from pain and discomfort. Osteochondromas can cause growth disturbances including madelung deformity (40$60 \%)$, unequal limb length (10-50\%) and joint deformity $(2-55 \%)^{3,4,5}$.

Histology of osteochondromas shows a strikingly consistent morphology. An osteochondroma consists of a bony stalk and a cartilaginous cap. The cap is lineated peripherally with the perichondrium, which is continuous with the periosteum of the underlying bone. The cortex of the stalk is in continuity with the cortex of the host bone, thus creating a continuous medullary cavity (Figure 1). The cap has the histological appearance of an epiphyseal growth plate with chondrocytes lined up in columns (Figure 2$)^{6-11}$.

Besides the uniform histological appearance, there is a uniform biological development. The osteochondromas develop when the patient grows and cease to develop when the patient reaches skeletal maturity. No new osteochondromas develop thereafter. The lesions enlarge at a growth rate proportionate to the overall growth of the patient ${ }^{7,12,13}$.

The direction of growth points away from the adjacent growth plate and away from the adjacent joint. The lesion grows at an average angle of 60 degrees to the normal growth direction of the bone $\mathrm{e}^{10,14}$. When an osteochondroma presents itself, it rarely spontaneously regresses during the course of childhood and puberty ${ }^{7,13,15,16}$. Next to the naturally arising osteochondromas, investigators were able to create osteochondromas surgically by inverting a span of the ring of LaCroix ${ }^{17}$. These surgically created osteochondromas all disappear eventually due to spontaneous regression ${ }^{17,18}$. Radiographically osteochondromas appear less dense and they can fracture spontaneously ${ }^{19-22}$. On clinical removal the osteochondromas appear to have a less dense, osteoporotic-like structure ${ }^{8}$. In some patients with $\mathrm{HMO}$ general osteoporosis is described ${ }^{23}$. 


\section{Chapter 5}

Several topics remain unclear in the development of osteochondromas, like why these osteochondromas do not all resorb or regress. Osteochondromas can disappear spontaneously and surgically developed osteochondromas all disappear. Why every osteochondroma does not disappear eventually? Especially taking into account that all osteochondromas grow at a 60-degree angle to the bone and therefore they are not in line with the axis of the bone and do not sustain normal mechanical loading. In normal bone, non-loaded bone tissue will be resorbed if it is not sufficiently loaded $^{24}$. It thus would be expected that osteochondromas eventually disappear as a result of osteoclast resorption ${ }^{10,25}$. Answers to the preservation from resorption questions might be found in the architecture of the osteochondroma. Presently, no quantitative data about the morphology of osteochondromas is available.

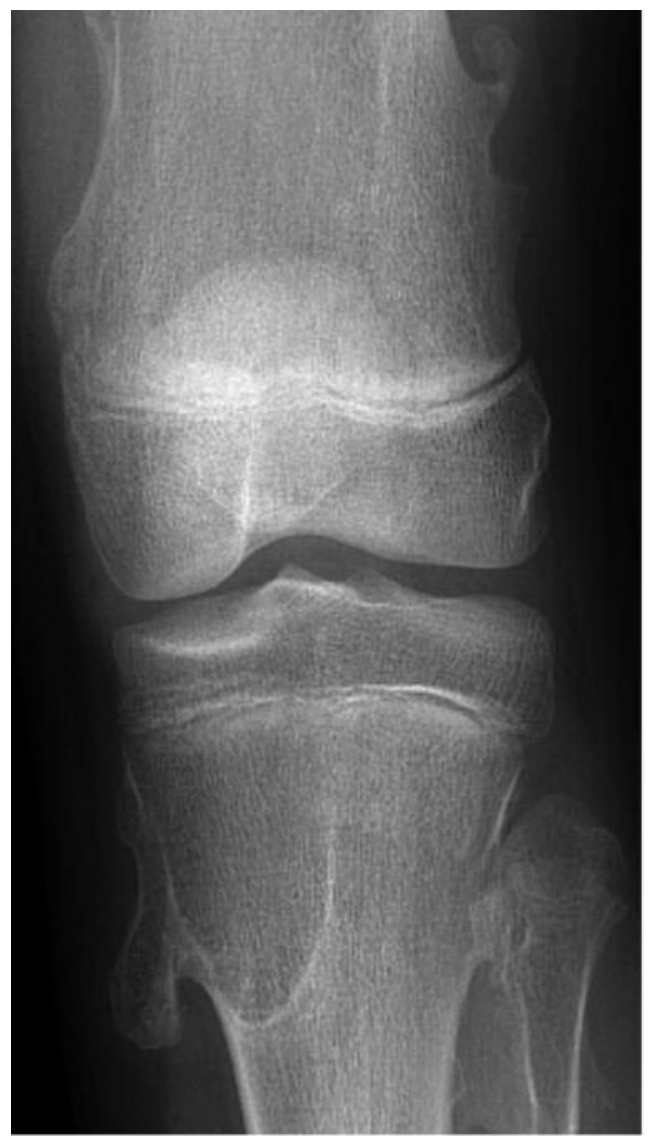

The aim of this research is to quantify the anatomical microstructure of osteochondromas. Because of their off-axis position it is expected that osteochondromas have a less developed, osteoporotic-like, microstructure. To investigate this, the bone morphological parameters of 9 osteochondromas were measured using micro-CT. The results were compared to histological samples and literature values.

Figure 1: AP radiograph of a knee of a 14year-old girl with $\mathrm{HMO}$, multiple osteochondromas are visible on the distal femur, the proximal tibia and the proximal fibula. 


\section{Materials AND Methods}

Fifteen surgically removed osteochondromas were selected. The osteochondromas were fixated in formalin. The size of the specimens varied between $15 \mathrm{~mm}$ up to 32 $\mathrm{mm}$ in length and all specimens were irregularly shaped. Micro-CT scans were performed using a vivaCT40 microCT-system (Scanco Medical, Brüttisellen, Switzerland). The specimens were scanned with an isotropic voxel size of $30 \mathrm{~m}$. The system operated at an energy level of $70 \mathrm{kV}$, and a current of $114 \mathrm{~A}$. After reconstruction, the bone was segmented from the images using a threshold of 200 per mile of the maximum value. The structural properties of each specimen were evaluated using the micro-CT system software. Contours were drawn to delineate

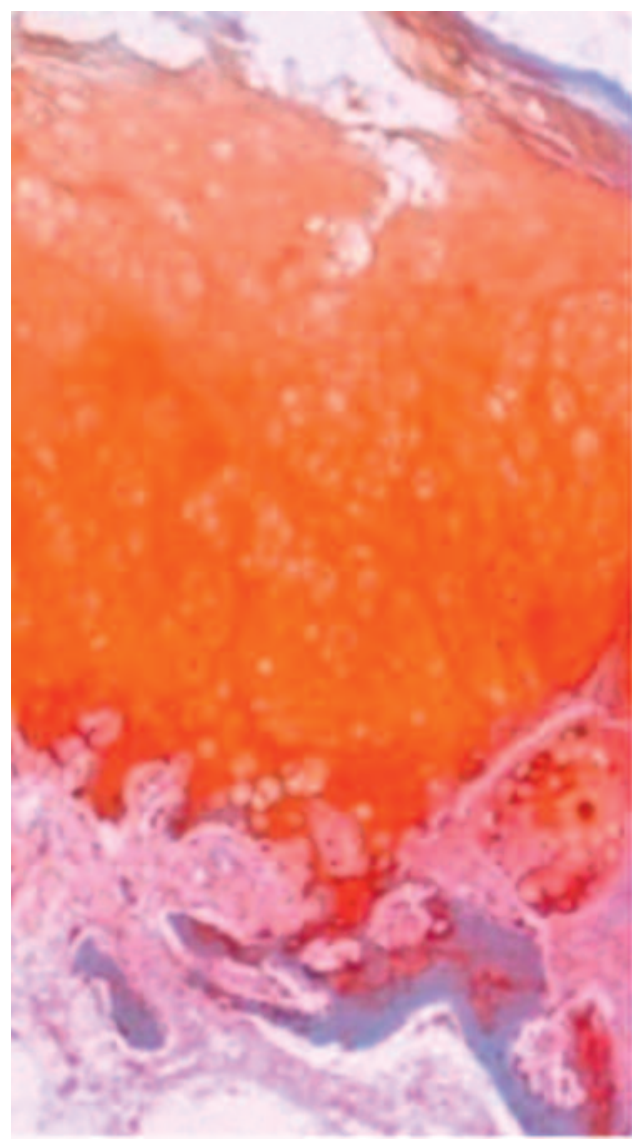
the region of the osteochondroma in the scan (Figure 3). For the first slice this was done manually, thereafter the system automatically determines the contouring of the next slices. When the automatic contouring deviated from the bony contour, the operator manually adjusted the contour.

Figure 2: Safranin-O staining of the cartilaginous cap of an osteochondroma, chondrocytes lined up in columns. 


\section{Chapter 5}

Of the fifteen samples only the samples with a total volume larger than $100 \mathrm{~mm}^{3}$ and a calcified size (bone not cartilage) of at least 4 by 4 by $4 \mathrm{~mm}$ were selected for evaluation because morphological measurements in smaller samples would be inaccurate. Therefore six samples were excluded. The nine samples for evaluation were derived from six different patients. The patients ( 4 females, 2 males) varied in age from 4 up to 16 years old (mean age 9 years). The places of origin of the osteochondromas were the distal ulna $(n=2)$, the scapula $(n=2)$ and the knee $(n=5)$. Of these nine samples the following parameters were quantified: volume fraction (BV/TV), trabecular separation (Tb.Sp, $\mu \mathrm{m})$, trabecular number $\left(\mathrm{Tb} . \mathrm{N}, \mathrm{mm}^{-1}\right)$, trabecular thickness (Tb.Th, $\mu \mathrm{m})$, mean density of the bone tissue volume (MDBV, $\mathrm{mg} \mathrm{HA} / \mathrm{ml}$ ), mean density of the total volume (MDTV, $\mathrm{mg} \mathrm{HA} / \mathrm{ml}$ ) and degree of anisotropy (DA). The mean trabecular thickness, separation and number of cancellous bone were calculated using direct 3D measure and are denoted here as Tb.Th", Tb.Sp ${ }^{*}$ and Tb. $\mathrm{N}^{*}$ respectively ${ }^{26,27,28}$. In order to compare our values with literature values, trabecular thickness, spacing and number were also evaluated using the plate model assumption and in that case denoted as Tb.Th\#, Tb.Sp\# and Tb.N\# respectively. The DA was defined as the ratio of the largest over the smallest eigenvalue of the Mean Intercept Length fabric tensor.

For a subset of five samples histology was performed to identify the cartilage on top of the osteochondromas. The samples were embedded in plastic, polymethylmethacrylate (PMMA, Technovit 9100, Hereaus-Kulzer, Germany). After polymerization, sections were stained according to Masson Goldner (Carl Roth, Germany) to clearly identify the trabeculae and the cartilage cap. Thereafter $50 \mathrm{~m}$ sections were generated using a saw microtome (SP 1600, Leica, Germany). Sections were analyzed and digitized by light microscopy (Axioscope A1, AxioVision LE release 4.8.2, Carl Zeiss, Germany). The central two slices were selected and in each slice 10 trabeculae were randomly selected and their thickness was measured.

To compare the outcome of the values from the osteochondromas a literature search was performed for bone parameters in normal children and adolescents. Four different articles on bone architecture were selected on the basis of the age group of the patients ${ }^{29-32}$. This selection was made because the osteochondromas were retrieved from children and adolescents. Adolescent bone has a different microarchitecture compared to adult cancellous bone with a lower bone surface 

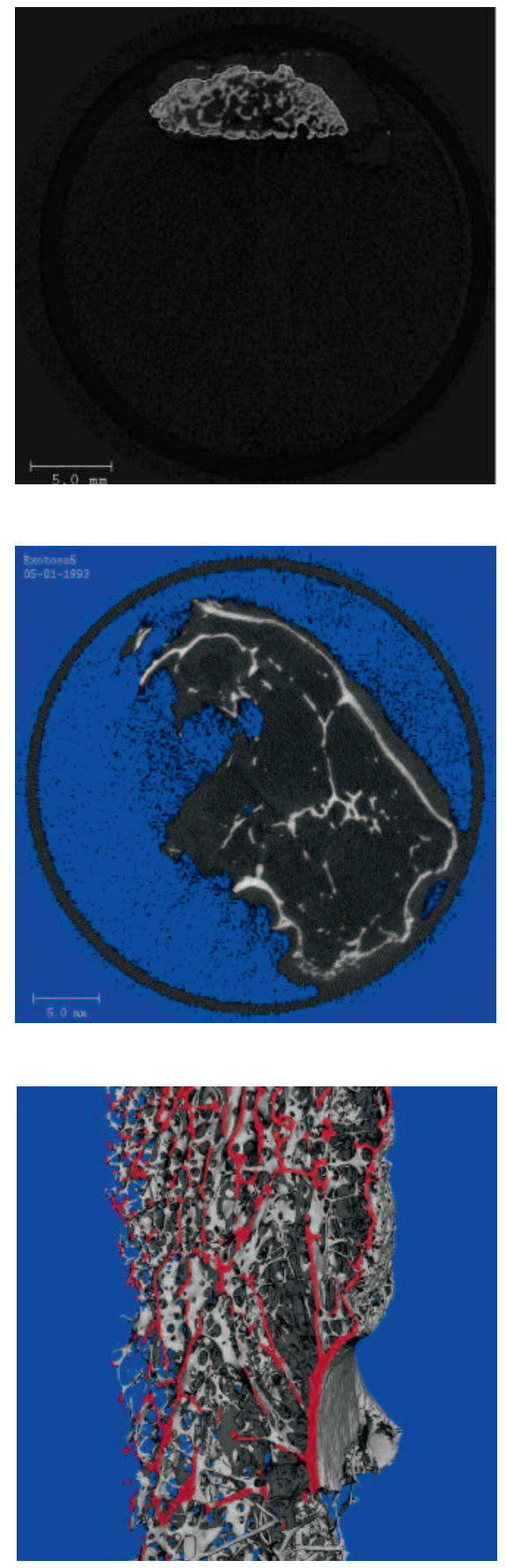

density, a greater trabecular separation and a lower trabecular number than adult bone $^{30}$. These literature studies involved bone taken from different sites: tibia, the non-dominant radius and the iliac-crest.

Figure 3a: Example of a Micro-CT image of a broad based osteochondroma.

Figure 3b: Example of a osteochondroma with a low trabecular number.

Figure 3c: Example of a 3D reconstruction of an osteochondroma cut in half to reveal the inner structure. The cut-surface is coloured red. 


\section{Chapter 5}

Approval of the local ethics committee was received for this protocol in May 2008, number MEC 08-4-028. An informed consent was obtained from all patients or their parents.

\section{Results}

Micro-CT scanning results show a broad variation in bone morphology for the different specimens, Coefficient of Variation (CV) values varying between $29 \%$ and $49 \%$ (Table 1). In spite of the large variation in structural parameters (Tb.Sp, Tb.N, $\mathrm{Tb} . \mathrm{Th}$,) the bone tissue mineralisation varied only in a very limited range with a CV of 2 .

\section{Histology}

All specimens showed a histological image identical to the known structure of an osteochondroma. A cartilage cap was visible as well as a trabecular configuration of bone. The average height of the cartilage cap was $1557 \mu \mathrm{m}$ (SD $738 \mu \mathrm{m})$ with a range of 572 to $2424 \mu \mathrm{m}$. The thickest cap was found in the sample from the youngest patient. All specimens showed a clear interdigitation from cartilage and the trabecular bone. The transition zone was irregularly shaped. The results of the trabecular thickness measurements are shown in table 1. The trabecular thickness

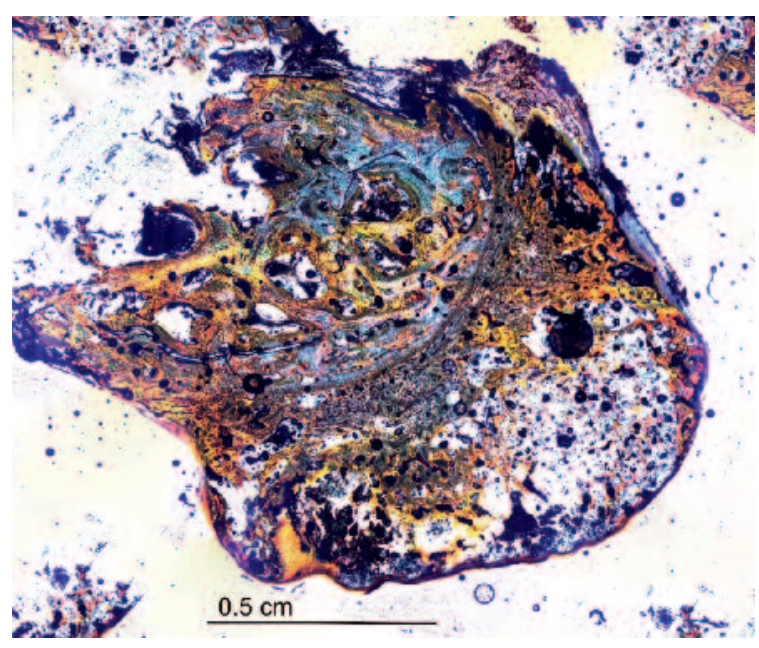
found in the selected trabeculae varies widely. The mean of trabecular thickness is higher than the values from the Micro-CT scans, but there are outliers on both sides of the spectrum.

Figure 4: Histological image of an osteochondroma, Masson-Goldner staining showing the irregular bone formation. 


\section{Values in literature}

The first author, the patients age group, the sites of the study and the values of the bone parameters are listed in table 2 . The values for 3D and plate model parameters are listed separately when available. The volume fraction values in all groups are comparable to the volume fraction found in our study, indicating that the osteochondromas have a bone volume fraction comparable to that of normal subjects in the same age group. The trabecular separation in the osteochondromas, however, is almost double that of the separation found in normal bone. Further the microarchitecture of the osteochondromas shows a relatively low trabecular number and a high trabecular thickness. As for the degree of anisotropy (DA) there was only one comparative study ${ }^{30}$ with an almost identical DA. In their study, however, samples of the proximal tibia of cadaver bones of children were measured.

\begin{tabular}{|c|c|c|c|c|c|}
\hline Parameter & Mean & Median & SD & Minimum - Maximum & $\mathrm{CV}(\%)$ \\
\hline BV/TV [\%] & 18 & 17 & 7 & $11-33$ & 39 \\
\hline $\operatorname{Tb} . S p^{*}[\mu \mathrm{m}]$ & 1254 & 1330 & 612 & $528-2412$ & 49 \\
\hline Tb. $N^{*}[m m-1]$ & 1.01 & 0.91 & 0.48 & $0.48-1.88$ & 48 \\
\hline Tb.Th* $[\mu \mathrm{m}]$ & 305 & 280 & 115 & $153-578$ & 38 \\
\hline Tb.Sp\# [um] & 989 & 1061 & 287 & $303-1332$ & 29 \\
\hline Tb.N\# [mm-1] & 0.94 & 0.84 & 0.46 & $0.58-2.15$ & 49 \\
\hline Tb.Th\# [um] & 205 & 194 & 72 & $132-382$ & 35 \\
\hline DA [-] & 1.52 & 1.29 & 0.40 & $1.23-2.43$ & 26 \\
\hline MDBV [mg HA/ml] & 784.53 & 787.43 & 17.04 & $764.06-811.82$ & 2 \\
\hline MDTV [mg HA/ml] & 158.57 & 138.90 & 64.66 & $91.04-297.30$ & 41 \\
\hline Tb.Th histology [um] & 543 & 632 & 158 & $22-3342$ & 29 \\
\hline
\end{tabular}

Table 1: The Median, mean, standard deviation and the range of the values of the structural parameter (*direct 3D measure, \# plate model). The values of the histological measurements are shown in the last line. 


\begin{tabular}{|c|c|c|c|c|c|c|}
\hline Author & Burrows & Zhu & Zhu & Ding & Kirmani & Staal \\
\hline Age & $15-20$ & $12-19$ & $12-19$ & $9-17$ & $6-21$ & $4-16$ \\
\hline Site & Tibia & Iliac crest & Iliac crest & Tibia & Radius & Osteochondroma \\
\hline Imaging & HR-pQCT & Micro-CT & Micro-CT & Micro-CT & HR- $p Q C T$ & Micro-CT \\
\hline Resolution $(\mu \mathrm{m})$ & 82 & 40 & 40 & 40 & 82 & 30 \\
\hline N & 278 & 15 & 16 & 6 & 127 & 9 \\
\hline BV/TV (\%) & 15 & 20 & 19 & 20 & 16 & 18 \\
\hline TB. $N^{*}\left(\mathrm{~mm}^{-1}\right)$ & 1.75 & 1.81 & - & 1.37 & 2.0 & 1.01 \\
\hline Tb.Th* $(\mu \mathrm{m})$ & 91 & 156 & - & 187 & 80 & 305 \\
\hline $\mathrm{Tb} . S p^{*}(\mu \mathrm{m})$ & 490 & 552 & - & 735 & 440 & 1254 \\
\hline TB.N\# $\left(\mathrm{mm}^{-1}\right)$ & - & - & 1.9 & - & - & 0.94 \\
\hline Tb.Th\# ( $\mu \mathrm{m})$ & - & - & 104 & - & - & 205 \\
\hline Tb.Sp\# ( $\mu \mathrm{m})$ & - & - & 479 & - & - & 989 \\
\hline DA (-) & & & & 1.54 & & 1.52 \\
\hline
\end{tabular}

Table 2: Mean values for the structural parameters of the different groups.

\section{Discussion}

Using micro-CT, the microarchitecture of osteochondromas of HMO-affected children was determined. In this pilot study the microarchitecture shows thicker and wider spaced trabeculae with a relatively normal trabecular number and a normal bone volume fraction when compared to normal bone of children in the same age range. In spite of the large variation in structural parameters, the bone tissue mineralisation varied only in a very limited range, suggesting that the biological processes that lead to mineralisation are not affected. The origin of thicker and wider spaced trabeculae of an osteochondroma might be explained by the presence of the cartilage cap on top of the osteochondroma. In HMO patients the organization of chondrocytes in the epiphysis, specifically that of the columnar structures, has been lost, suggesting changes in cell adhesion and motility and related cell-matrix and cell-cell communication mechanisms ${ }^{33}$. The cartilage cap has a similar structure to an epiphysis. It consists of separated clusters of cartilage cells in columns, similar to 
a lobbed epiphysis. The cartilage cap chondrocytes are separated in different cell clusters. These separated clusters eventually mineralize and if each cluster of chondrocyte cells produces their own set of trabeculae, it might lead to thicker and wider spaced trabeculae.

Besides the cartilage cap-architecture, the cap can also have a biological/paracrine effect on the bone below it, by influencing the activity of bone remodelling ${ }^{18}$. In HMO the exostosin (EXT) genes are involved, leading to a heparan sulfate deficiency influencing growth plate-associated signalling proteins ${ }^{33-35}$. Sgariglia et al. described a significantly larger number of osteoclasts and deranged trabecular bone formation in both extent and cell composition in Ext1 deficient mice $^{36}$. The abnormal biological environment lacking heparan sulfate and influencing the osteoclasts, amongst other, is expected to disturb the normal bone remodelling influencing the microarchitecture. The observed increased trabecular spacing may be responsible for the clinical appearance of an osteoporotic-like structure of the removed osteochondromas ${ }^{8}$, the wider spaced trabeculae giving it a visual osteoporotic appearance. Possibly, these thicker but wider spaced trabeculae can carry less load and occasionally break, explaining the spontaneous fractures ${ }^{19-22}$.

The histology showed the cartilage cap and the bony structures, the typical build of an osteochondroma. The measured trabeculae were on average thicker than the micro-CT values (table 1), but the measurements had a wider variation (Tb.Th micro-CT average $205 \mu \mathrm{m}$ plate model, variation 132-382, CV 35 and Tb.Th histology average $543 \mu \mathrm{m}$, variation 22-3342, CV 29). This might be due tot the selection procedure. The selected histological slices were all evaluated in the transition zone between the cartilage and the bone formation. The micro-CT measurements were done in the complete osteochondroma and not only in the transition zone. In the transition zone the trabeculae originate and might therefore be more variable in size. Also this zone shows multiple fine indentations just below the cartilage cap and might therefore not be well presented in the micro-CT measurements.

This pilot study sets a first step in elaborating the architectural properties of osteochondromas. However there are limitations. Of the initial 15 samples 6 were not calcified enough, leaving only a small number for evaluation. The age of the patients of the evaluated samples varied widely as did the place of origin. There is 


\section{Chapter 5}

not enough reference to compare the findings to normal bone in these age groups and there are no values to compare the osteochondromas to. For all determined parameters more research is needed with a greater specimen population to confirm the outcomes. Future studies with Extreme CT evaluation can be performed on osteochondromas in vivo in order to compare the osteochondroma bone morphology to the morphology of the adjacent long bone and to study the trabecular osteochondroma development in time.

To our knowledge, this is the first study done with micro-CT derived data and human osteochondromas for this purpose.

\section{CONCLUSION}

This study is the first to show that, compared to that of age matched controls, the microarchitecture of the osteochondromas shows less but thicker trabeculae that are wider separated with a normal bone volume fraction. The microarchitecture only resembles an osteoporotic structure in some aspects (increased trabecular spacing, decreased trabecular number). The structure may be due to the biological influence of the cartilage cap nearby.

\section{Conflict of interest}

Bert van Rietbergen works as a consultant for Scanco Medical, Switzerland. No funding was received for this research. 


\section{REFERENCES}

1. Khurana J, F. FA-K, Bovee J (2002) Osteochondroma. World Health Organisation Classification of tumours Patholog and Genetics of Tumours of Soft Tissue and Bone 234236.

2. Bovee J, Hogendoorn PC (2002) MultipleOsteochondromas. World Health Organisation Classification of tumours Pathology and Genetics of Tumours of Soft Tissue and Bone: 260362.

3. Schmale GA, Conrad EU, 3rd, Raskind WH (1994) The natural history of hereditary multiple exostoses. J Bone Joint Surg Am 76: 986-992.

4. Legeai-Mallet L, Munnich A, Maroteaux P, Le Merrer M (1997) Incomplete penetrance and expressivity skewing in heredita multiple exostoses. Clin Genet 52: 12-16.

5. Wicklund CL, Pauli RM, Johnston D, Hecht JT (1995) Natural history study of hereditary multiple exostoses. Am J Med Genet 55: 43-46.

6. Peterson HA (1989) Multiple hereditary osteochondromata. Clin Orthop Relat Res: 222230.

7. Hennekam RC (1991) Hereditary multiple exostoses. J Med Genet 28: 262-266.

8. Kitsoulis P, Galani V, Stefanaki K, Paraskevas G, Karatzias G, Agnantis NJ, Bai M. (2008) Osteochondromas: review of the clinical, radiological and pathological features. In Vivo 22: 633-646.

9. Hameetman L, Bovee JV, Taminiau AH, Kroon HM, Hogendoorn PC (2004) Multiple osteochondromas: clinicopathological and genetic spectrum and suggestions for clinical management. Hered Cancer Clin Pract 2: 161-173.

10. Milgram JW (1983) The origins of osteochondromas and enchondromas. A histopathologic study. Clin Orthop Relat Res: 264-284.

11. Huegel J, Sgariglia F, Enomoto-Iwamoto M, Koyama E, Dormans JP, Pacifici M. (2013) Heparan sulfate in skeletal development, growth, and pathology: the case of hereditary multiple exostoses. Dev Dyn 242: 1021-1032.

12 Bovee JV (2008) Multiple osteochondromas. Orphanet J Rare Dis 3: 3.

13. Solomon, L. (1964). "Hereditary Multiple Exostosis." Am J Hum Genet 16: 351-363.

14. Vanhoenacker FM, Van Hul W, Wuyts W, Willems PJ, De Schepper AM (2001) Hereditary multiple exostoses: from genetics to clinical syndrome and complications. Eur J Radiol 40: 208-217. 


\section{Chapter 5}

15. Yanagawa T, Watanabe H, Shinozaki T, Ahmed AR, Shirakura K, Takagishi K. (2001) The natural history of disappearing bone tumours and tumour-like conditions. Clin Radiol 56: 877- 886.

16. Hoshi M, Takami M, Hashimoto R, Okamoto T, Yanagida I, Matsumura A, Noguchi K. (2007) Spontaneous regression of osteochondromas. Skeletal Radiol 36: 531-534.

17. Delgado E, Rodriguez JI, Rodriguez JL, Miralles C, Paniagua R (1987) Osteochondroma induced by reflection of the perichondrial ring in young rat radii. Calcif Tissue Int 40: 85-90.

18. Trebicz-Geffen M, Nevo Z, Evron Z, Posternak N, Glaser T, Fridkin M, Kollander Y, Robinson D. (2003) The short-lived exostosis induced surgically versus the lasting genetic hereditary multiple exostoses. Exp Mol Pathol 74: 40-48.

19. Henry NE, Carow SD, Kocher BL (2014) Osteochondromfracture at the distal medial femur. J Orthop Sports Phys Ther 44: 457.

20. Kanauchi T, Suganuma J, Kawasaki T, Mochizuki R, Inoue Y, Uchikawa S, Kitamura K, Honda A. (2012) Fracture of an osteochondroma of the femoral neck caused by impingement against the ischium. Orthopedics 35: e1438-1441.

21. Kose O, Ertas A, Celiktas M, Kisin B (2009) Fracture of an osteochondroma treated successfully with total excision: two case reports. Cases J 2: 8062.

22. Theodorou SD, Karamitsos S, Tsouparopoulos D, Hatzipavlou AG (1978) Rare complications of exostosis. Fractures and injury to the common peroneal nerve. Acta Orthop Belg 44: 496-505.

23. Lemos MC, Kotanko P, Christie PT, Harding B, Javor T, Smith C, Eastell R, Thakker RV. (2005) A novel EXT1 splice site mutation in a kindred with hereditary multiple exostosis and osteoporosis. J Clin Endocrinol Metab 90: 5386-5392.

24. Wolff J (1986 (translation of the German 1892 edition)) "The Law of Bone Remodeling" Berlin Heidelberg New York: Springer

25. Chin KR, Kharrazi FD, Miller BS, Mankin HJ, Gebhardt MC (2000) Osteochondromas of the distal aspect of the tibia or fibula. Natural history and treatment. J Bone Joint Surg Am 82: 1269-1278.

26. Adams JE (2009) Quantitative computed tomography. European Journal of Radiology 71: 415-424.

27. Gasser JA, Ingold P, Grosios K, Laib A, Hammerle S, Koller B. (2005) Noninvasive monitoring of changes in structural cancellous bone parameters with a novel prototype micro-CT. J Bone Miner Metab 23 Suppl: 90-96. 
28. Chappard C, Marchadier A, Benhamou L (2008) Interindividual and intraspecimen variability of 3-D bone microarchitectural parameters in iliac crest biopsies imaged by conventional micro-computed tomography. J Bone Miner Metab 26: 506-513.

29. Burrows M, Liu D, McKay H (2010) High-resolution peripheral QCT imaging of bone micro-structure in adolescents. Osteoporos Int 21: 515-520.

30. Ding M, Danielsen CC, Hvid I, Overgaard S (2012) Three- dimensional microarchitecture of adolescent cancellous bone. Bone 51: 953-960.

31. Kirmani S, Christen D, van Lenthe GH, Fischer PR, Bouxsein ML, McCready LK, Melton LJ 3rd, Riggs BL, Amin S, Müller R, Khosla S. (2009) Bone structure at the distal radius during adolescent growth. J Bone Miner Res 24: 1033-1042.

32. Zhu F, Qiu Y, Yeung HY, Lee KM, Cheng CY (2009) Trabecular bone micro-architecture and bone mineral density in adolescent idiopathic and congenital scoliosis. Orthop Surg 1: 7883.

33. De Andrea CE, Wiweger M, Prins F, Bovee JV, Romeo S, Hogendoorn PC. (2010) Primary cilia organization reflects polarity in the growth plate and implies loss of polarity and mosaicism in osteochondroma. Lab Invest 90: 1091-1101.

34. Benoist-Lasselin C1, de Margerie E, Gibbs L, Cormier S, Silve C, Nicolas G, LeMerrer M, Mallet JF, Munnich A, Bonaventure J, Zylberberg L, Legeai-Mallet L. (2006) Defective chondrocyte proliferation and differentiation in osteochondromas of MHE patients. Bone. 39(1):17-26.

35. Staal HM, Witlox AMA, Mooij DT, Emans PJ, Ham SJ, Van Rhijn LW, Welting TMJ. (2014) Current Knowledge on Exostoses Formation in Hereditary Multiple Exostoses: Where do Exostoses Originate and in What Way is their Growth Regulated?. Hereditary Genet 3: 3.

36. Sgariglia F, Candela ME, Huegel J, Jacenko O, Koyama E, Yamaguchi Y, Pacifici M, Enomoto-Iwamoto M. (2013) Epiphyseal abnormalities, trabecular bone loss and articular chondrocyte hypertrophy develop in the long bones of postnatal Ext1-deficient mice. Bone 57: 220-231. 


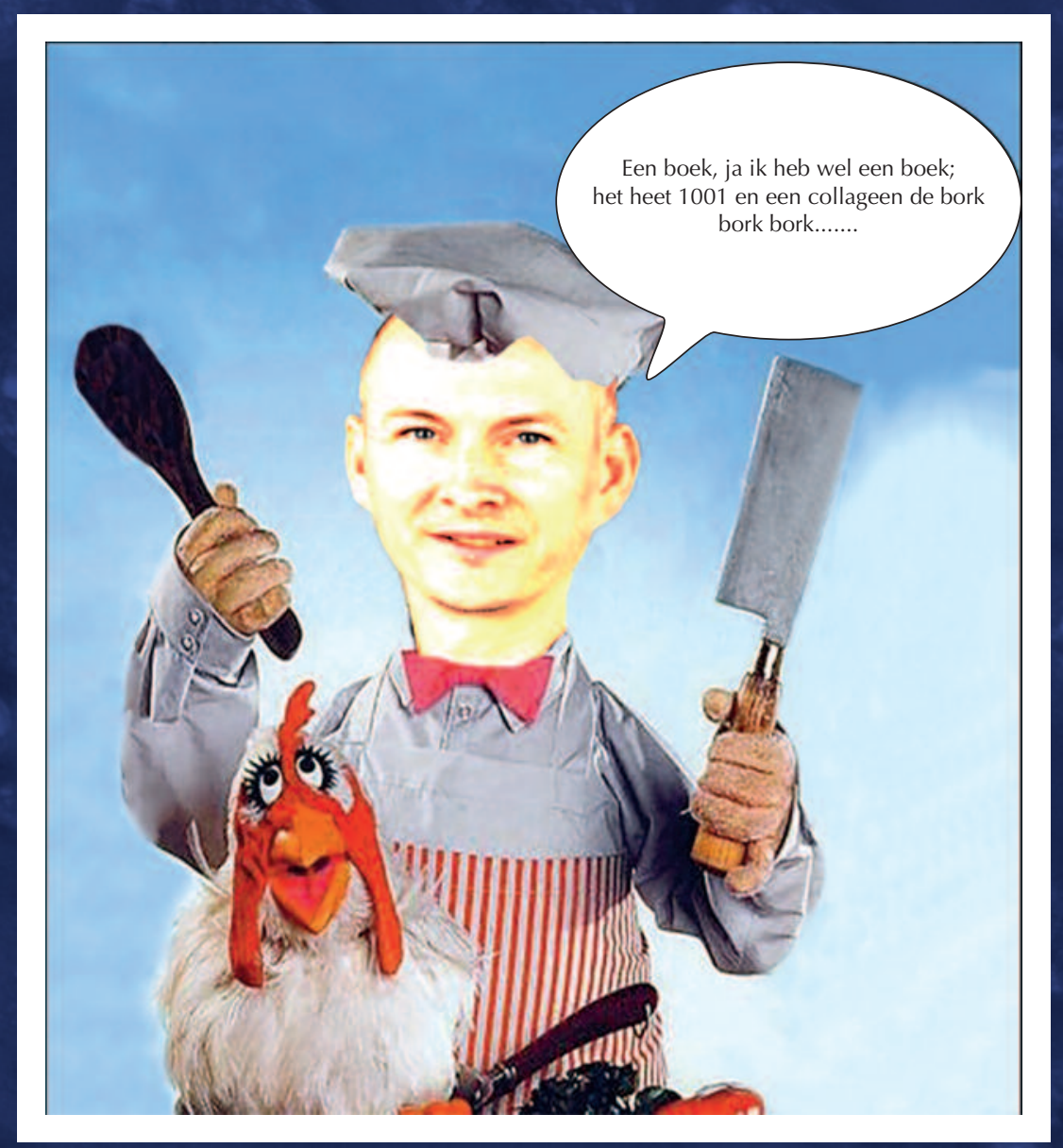




\section{ChAPTER SIX}

\section{Dysplasia Epiphysealis Hemimelica: an histological comparative study with osteochondromas}

Jasper Stevens, Tim J.M. Welting, M. Adhiambo Witlox, Lodewijk W. van Rhijn, Heleen M. Staal 


\section{Chapter 6}

\section{Abstract}

Dysplasia Epiphysealis Hemimelica (DEH) also known as Trevor's Disease is a rare developmental disorder resulting in overgrowth of the epiphysis. Based on histological evaluation, it is often described as an osteochondroma or an osteochondroma-like lesion, although there are clinical and genetic differences between $\mathrm{DEH}$ and osteochondromas. To investigate the hypothesis that $\mathrm{DEH}$ and osteochondromas are histologically identical, two cases of DEH and two cases of osteochondromas in patients with Hereditary Multiple Osteochondroma $(\mathrm{HMO})$ are compared at histological level.

Histologically, clumping of chondrocytes in a fibrillar matrix, a thick disorganized cartilage cap and ossification centres with small amounts of unresorbed cartilage were observed in DEH. In contrast, chondrocyte organisation in cartilage of osteochondromas displays characteristics of the normal growth plate. To investigate the differences in cell type involvement, immunohistochemistry was performed in DEH and osteochondromas. It showed differences in expression of collagen type II, collagen type $\mathrm{X}$ and Sox9. Collagen type II was expressed in the extracellular matrix surrounding proliferative and hypertrophic chondrocytes in osteochondromas, while weak expression was observed in the entire cartilage cap in DEH. Collagen type $X$ was not expressed in $\mathrm{DEH}$, while expressed in the pericellular matrix surrounding hypertrophic chondrocytes in osteochondromas. Staining for Sox9 was positive in the hypertrophic chondrocytes in osteochondromas, while expressed in the nuclei of all chondrocyte clusters in DEH.

In conclusion, both morphological and immunohistological differences were observed in histological sections of DEH and osteochondromas. This result supports the earlier observed clinical, radiological and genetic differences and implies a different aetiology between DEH and osteochondroma formation. 


\section{INTRODUCTION}

Dysplasia Epiphysealis Hemimelica (DEH), also known as Trevor's disease, is a rare developmental disorder characterized by asymmetric enlargement of the epiphyseal cartilage of the long bones. It was first described in 1926 by Mouchet and Belot, who initially named it Tarsomegalia ${ }^{1}$. In 1950, Trevor reported a series of 8 cases and called it tarso-epiphyseal aclasis ${ }^{2}$. Finally, it was named Dysplasia Epiphysealis Hemimelica by Fairbank in $1956^{3}$.

$\mathrm{DEH}$ is characterized by cartilaginous overgrowth of the epiphysis of long bones. It is usually restricted to one side of the epiphysis (hemimelic). The medial side is twice as often affected compared to the lateral side. In addition, the lower extremity is approximately three times more often affected and involvement of multiple joints is more common in the lower extremity. The ankle and wrist are the most affected joints for the lower and upper extremity respectively ${ }^{4}$. Nowadays, DEH is classified based on the number of affected epiphyses, i.e. "localized" when only one epiphysis is affected, "classic" when more epiphyses are affected in the same limb and "generalized" when all epiphyses in the entire extremity are affected ${ }^{5}$. DEH is usually diagnosed in children between two and eight years old and it is three times more often diagnosed in boys ${ }^{4,5}$.

The most reported complaints at first clinical presentation are pain, limitation in range of motion of the affected joint, and deformity or swelling of the affected joint. However, a valgus or varus deformity or a leg-length discrepancy can also be the presenting symptom. Radiography is useful for the diagnosis of DEH, while CT and MRI are useful in the preoperative planning ${ }^{6}$. Treatment of symptomatic lesions consists of surgical resection of the lesion, resulting in good long-term results. An expectative policy with follow up is justifiable in asymptomatic lesions, since malignant transformation is not reported ${ }^{7}$.

DEH belongs to the group of benign skeletal osteocartilaginous tumours and is often described as an epiphyseal osteochondroma or as an osteochondroma-like lesion. This name suggests a common aetiology between DEH and osteochondromas. However, osteochondromas are common benign tumours, with an incidence of approximately $30-50 \%$ of all benign bone tumours, while DEH is extremely rare. An osteochondroma is a cartilage capped bony projection on the 


\section{Chapter 6}

external surface of the metaphysis of a bone and has a bone marrow cavity. The bone marrow cavity of the osteochondroma is continuous with the cavity of the underlying bone. Osteochondromas develop during the first decade of life; they cease to grow when the growth plates close and are often asymptomatic. Pain, bone deformities, bursa formation, arthritis and impingement of adjacent tendons, nerves or vessels are the most reported complaints ${ }^{8}$. Osteochondromas are easily diagnosed on conventional radiographs and symptomatic osteochondromas are often surgically resected. The most important complication of osteochondromas is malignant transformation. As stated earlier, occurrence of multiple osteochondromas is common, with Hereditary Multiple Osteochondroma ( $\mathrm{HMO})$ as underlying cause in $10-15 \%$ of the people with osteochondromas ${ }^{9-11}$. DEH is a very rare disease in contrast to $\mathrm{HMO}$, with a reported incidence of $1: 1,000,000$ and $1: 50,000$ respectively ${ }^{12,13}$.

DEH and osteochondromas are seen as different entities based on their different location of appearance (i.e. epiphysis versus metaphysis) and different clinical presentation. However, histopathological evaluation insinuates a common aetiology since DEH is often described as an osteochondroma-like lesion ${ }^{14-22}$. Therefore, the aim of this study is to test the hypothesis that DEH and osteochondromas are histologically identical diseases. We will report histological sections of two patients with DEH and compare them with histological sections of two age and gender matched patients with multiple osteochondromas, caused by $\mathrm{HMO}$, to investigate this hypothesis.

\section{Materials AND Methods}

Two patients with a histopathologically confirmed diagnosis of DEH were identified from the patient files of the department of Orthopaedic Surgery of the Maastricht University Medical Centre+. Both subjects underwent surgery for symptomatic DEH. These patients were compared with two age and gender matched patients diagnosed with $\mathrm{HMO}$, who also underwent surgery for symptomatic osteochondromas. 


\section{Tissue sampling and processing}

Tissue was available for all included patients at the start of this study. The local ethics committee approved the use of this tissue for this study (number MEC 08-4-028, May 2008). In addition, written informed consent was obtained from all patients and/or their parents. All specimens were fixed in $4 \%$ buffered formalin for at least 72 hours. After rinsing with tap water, tissues were decalcified with 10\% EDTA solution for up to 6 weeks. Sections of $5 \mu \mathrm{m}$ were cut from paraffin-embedded tissue and placed on glass slides.

\section{Immunohistochemistry}

After deparaffinization and rehydration, sections were treated with hyaluronidase or citrate according to the antibody-specific regimens to improve immunoreactivity (see Table 1). Subsequently, endogenous peroxidase activity was blocked and sections were rinsed with phosphate-buffered saline. Subsequently, sections were incubated with the primary antibody. Details of the antibodies and negative controls are shown in Table 1. Samples were incubated with an anti-mouse secondary antibody labelled with Horseradish Peroxidase (HRP) (Dako, Glostrup, Denmark). Adding of diaminobenzidine (DAB) solution resulted in visualizations of the antigens of interest. Hematoxylin was used for counterstaining of the sections. In addition, a Safranin-O staining was performed according to Caron et $\mathrm{a}^{23}$. Sections were analyzed and digitized by light microscopy (Axioscope A1, AxioVision LE release 4.8.2, Carl Zeiss, Germany).

\begin{tabular}{|c|c|c|c|c|c|c|}
\hline Antigen & Manufacturer & Antigen retrieval & Incubation time & Dilution & Secondary antibody & Negative control \\
\hline Collagen II & Developmental & Hyaluronidase & One hour at & $1: 200$ & Dako Envision & Monoclonal lgG1 \\
\hline monoclonal & studies by & $(4 \mathrm{mg} / \mathrm{ml}), 30$ & room & & HRP mouse & \\
\hline (Col II6B3) & Hybridoma Bank & minutes at $37^{\circ} \mathrm{C}$ & temperature & & & \\
\hline Collagen X & Quartett & Hyaluronidase & Overnight & $1: 25$ & Dako Envision & Monoclonal lgG1 \\
\hline monoclonal & & $(4 \mathrm{mg} / \mathrm{ml}), 30$ & at $4^{\circ} \mathrm{C}$ & & HRP mouse & \\
\hline (ColX53) & & minutes at $37^{\circ} \mathrm{C}$ & & & & \\
\hline Sox 9 & Santa Cruz & Citrate buffer, & Overnight & $1: 50$ & Dako Envision & Monoclonal lgG2 \\
\hline monoclonal & Biotechnology & boiled, 30 & at $4^{\circ} \mathrm{C}$ & & HRP mouse & \\
\hline (sc-166505) & Inc. & minutes & & & & \\
\hline
\end{tabular}

Table 1. Details of used antibodies and protocols 


\section{Chapter 6}

\section{RESULTS}

\section{Case descriptions}

In order to compare DEH and osteochondromas, two age and gender matched patients with $\mathrm{HMO}$ were included in this study. Clinical data of these $\mathrm{HMO}$ patients and included patients with DEH are reported in Table 2. Because of the rarity of $D E H$, a brief description of the patients with $\mathrm{DEH}$ will be given.

\section{Case $1 \mathrm{DEH}$}

An eight-year-old boy visited our outpatient clinic with pain and swelling of his left ankle. The pain progressed over several months and resulted in sparing of the left ankle during normal daily activities. There was no history of trauma or overuse. Family history was positive for M. Scheuermann.

During physical examination, an antalgic gait with sparing of the left ankle was observed. Inspection revealed a swelling of the anterior talocrural joint. There was no leg length discrepancy. Passive dorsiflexion of the left ankle was restricted to 0 degrees and resulted in pain. The boy experienced tenderness over the left musculus extensor hallucis longus. The range of motion of the left knee and left hip was unlimited.

Radiographs of the left ankle showed overgrowth of the medial epiphysis of the distal tibia, resulting in joint space narrowing between the distal tibia and talus. Furthermore, sclerosis of the talus and distal epiphysis of the tibia were visible. In addition, two centres of ossification were visible dorsally in the lateral radiograph (Figure 1A, white arrows). Magnetic resonance imaging (MRI) of the left ankle confirmed the overgrowth of the left distal tibial epiphysis and showed tibiotalar articular incongruence. High signal intensity was observed in the bony overgrowth of the left distal tibial (Figure1B, white arrow). In addition, thickening of the articular cartilage of the left talotibial joint is also visible.

During surgery, both the cartilage capped bony protuberance of the anterior distal tibia and the posteriorly located osteocartilaginous structure was resected (Figure 1C, white arrows). Intra-operative physical examination showed a dorsiflexion of 15 degrees. 

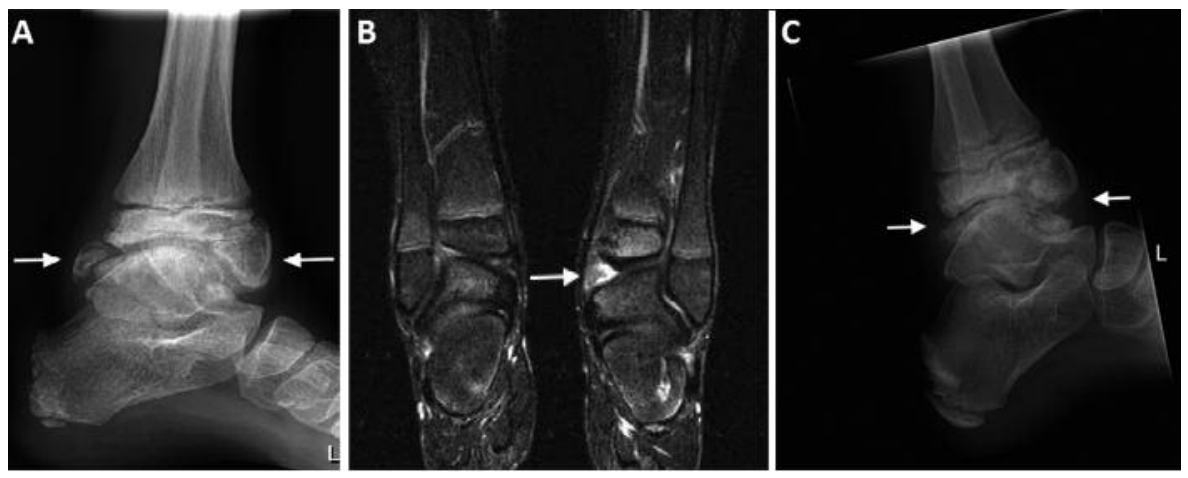

Figure 1. A Pre-operative lateral conventional radiograph of the left ankle; B Pre-operative coronal STIR MRI scan (TR 2000/TE 55) of both ankles; C Post-operative lateral conventional radiograph of the left ankle.

\section{Case 2 DEH}

The second case of DEH was a 3-year-old boy, who visited our outpatient clinic with pain in his left knee. The pain progressed over several months, resulting in an antalgic gait and pain of the left hip. The boy had no relevant medical history and there was no preceding trauma. Family history was negative for bone deformations, joint problems or dysplasia.

No abnormalities were observed during inspection of the feet, knee, hip and spine. A gait disturbance was present, with the patient keeping his left knee straight during the entire gait cycle. There was no limitation in range of motion, but pain was provoked during examination of the left knee. An evident swelling was palpated at the lateral side of the left knee joint. Due to provoked pain, the patient did not tolerate further physical examination of his left knee.

Two calcified regions were visible in the lateral region of the cartilaginous epiphysis of both the distal femoral and proximal tibia of the left knee in the conventional radiographs (Figure 2A, white arrows). A MRI of the left knee confirmed these observations and showed epiphyseal overgrowth (Figure 2B and 2C, white arrows).

The cartilage overgrowth of the lateral femoral epicondyle of the left knee was resected during surgery. Cartilaginous thickening of the tibial plateau was observed intra-operatively, but was not resected by the surgeons. 


\section{Chapter 6}
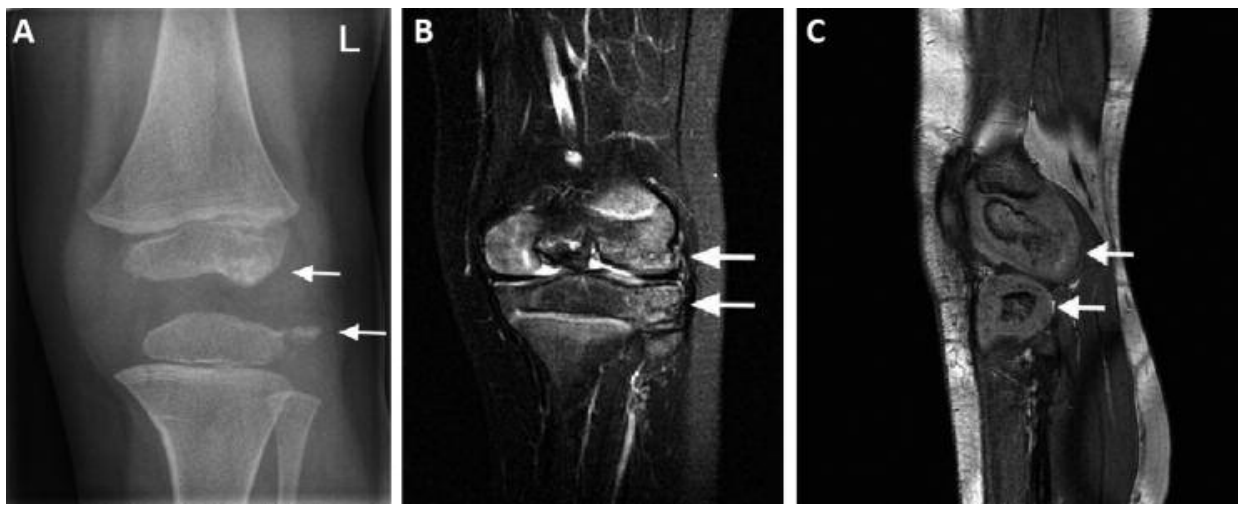

Figure 2. A Pre-operative anteroposterior radiograph of the left knee; B Pre-operative coronal T2W MRI scan (TR2968/TE70); C Pre-operative sagittal BFFE CART MRI scan (TR17/TE4).

\begin{tabular}{|c|c|c|c|c|}
\hline Disease & Age (years)/ sex & Clinical data & Location & Radiology \\
\hline DEH Case 1 & 9/ Male & $\begin{array}{l}\text { - Progressive pain. } \\
\text { - Swelling of the left ankle. }\end{array}$ & Left distal tibia. & $\begin{array}{l}\text { - Overgrowth of the epiphysis. } \\
\text { - Joint space narrowing between } \\
\text { tibia and talus. } \\
\text { - Two dorsal centres of ossification. }\end{array}$ \\
\hline HMO Case 1 & 8/ Male & $\begin{array}{l}\text { - Limitation during } \\
\text { daily activities. } \\
\text { - Aesthetic burden. } \\
\text { - Positive family history } \\
\text { for HMO. }\end{array}$ & $\begin{array}{l}\text { Left distal } \\
\text { femur*. }\end{array}$ & $\begin{array}{l}\text { - Scapula inferior }{ }^{\#} \text {, proximal humerï, } \\
\text { distal radius }{ }^{\#} \text {, distal ulna", proximal } \\
\text { femur }{ }^{\#} \text {, left SI-joint }{ }^{\$} \text {, left } \\
\text { acetabulum rim\$, } 3^{\text {th }} \text { and } 4^{\text {th }} \text { rib } \\
\text { right }{ }^{\$} \text {. }\end{array}$ \\
\hline DEH Case 2 & 3/ Male & $\begin{array}{l}\text { - Progressive pain } \\
\text { left knee. } \\
\text { - Palpable swelling lateral } \\
\text { knee joint. }\end{array}$ & $\begin{array}{l}\text { Left distal femur } \\
\text { and proximal } \\
\text { tibia. }\end{array}$ & $\begin{array}{l}\text { - Overgrowth and ossifications in } \\
\text { epiphysis of the distal femur and } \\
\text { proximal tibia. }\end{array}$ \\
\hline HMO Case 2 & 3/ Male & $\begin{array}{l}\text { - Progressive painless } \\
\text { deformity left forearm. } \\
\text { - Positive family history } \\
\text { for HMO. }\end{array}$ & $\begin{array}{l}\text { The left } \\
\text { distal ulna*. }\end{array}$ & $\begin{array}{l}\text { - Proximal humeri", distal femur\#, } \\
\text { proximal fibula", distal fibula", right } \\
\text { scapula }{ }^{\$} \text {, left proximal tibia }{ }^{\$} \text { and } \\
\text { left distal ulna } \$\end{array}$ \\
\hline
\end{tabular}

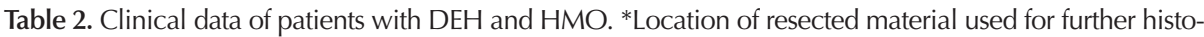
logical evaluation. "Bilateral occurrence of osteochondromas. ${ }^{\$}$ Unilateral occurrence of osteochondroma. 


\section{Histological evaluation}

Sections were routinely stained with Safranin-O to detect the presence of proteoglycans in the cartilage cap. Staining showed presence of proteoglycans in both osteochondroma and DEH (Figure 3A-C). The sections with osteochondromas showed a characteristically lobulated morphology of cartilage (Figure 3A). The osteochondroma tended to show characteristics of normal growth plate architecture, with chondrocytes orientated in columns with different zones of maturation (i.e. proliferative chondrocytes (white arrow) beneath the perichondrium and hypertrophic chondrocytes (black arrow) above the zone of ossification) (Figure 3B). However, zones of maturation are less organized compared with normal growth plates. The entire cartilage cap beneath the perichondrium showed intense SafraninO staining.

In contrast, chondrocytes are arranged in disorganised cell clusters in DEH. These clusters have a high density of chondrocytes with a relatively smaller cell volume. The most intense staining was detected in the pericellular matrix (Figure 3C).

\section{Collagen type II}

Specimens were stained for the presence of collagen type II in order to investigate the presence of this common collagen in the cartilage cap. Both osteochondromas and DEH showed clear expression for collagen type II (Figure 3D-F). No signal was detected in negative controls. An uneven distribution of collagen type II expression was observed in the cartilage matrix of osteochondromas. The most intense staining was observed in extracellular matrix surrounding proliferating and hypertrophic chondrocytes, with faint expression in the resting zone (Figure 3D).

In contrast to the osteochondromas, a more hyaline articular organisation of cartilage was observed in DEH. Faint, homogenous expression of collagen type II was observed with slightly increased expression beneath the perichondrium (Figure $3 \mathrm{E}$, white arrow). More intense expression of collagen type II was detected in the extracellular matrix surrounding the clusters of chondrocytes (Figure 3F, white arrow). In addition, the ossification centre with active endochondral ossification is clearly visible (Figure 3E). No signal was detected in negative controls (Fig 3G). 


\section{Chapter 6}

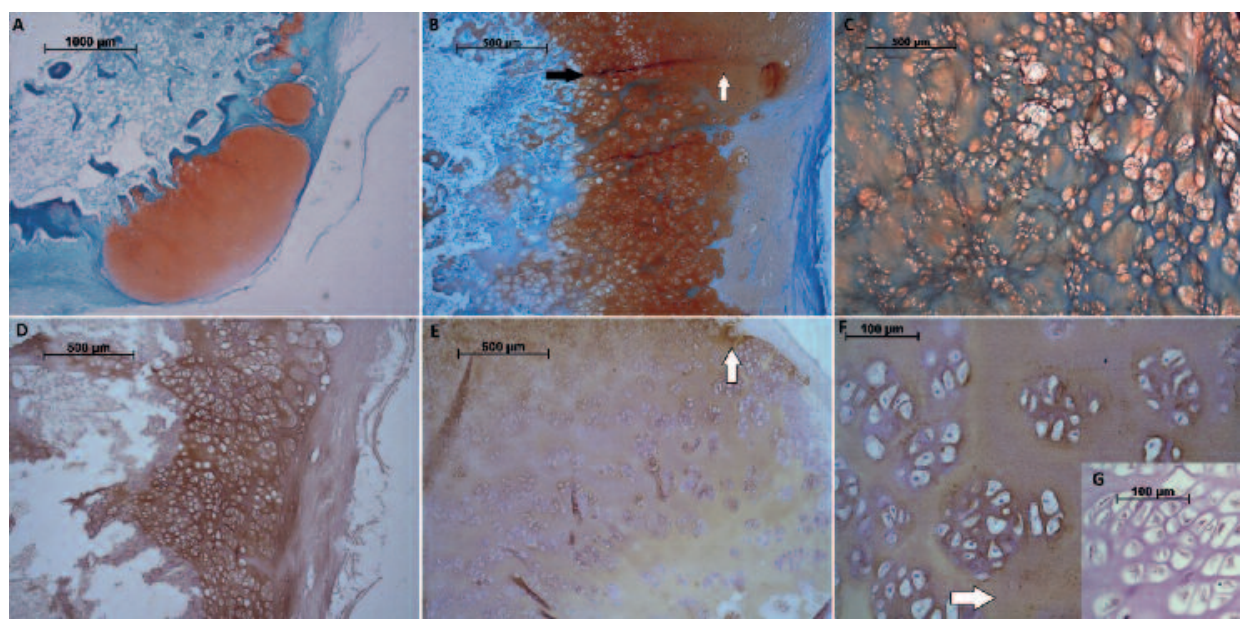

Figure 3. A-C Safranin-O staining in osteochondroma and DEH; D-G Collagen type II staining in osteochondroma and DEH; A) Positive staining of cartilage in osteochondroma; B)Positive staining of Safranin-O in osteochondroma, with chondrocytes arranged in columns; C) Pericellular staining for Safranin-O in DEH; D)Positive staining of collagen type II in osteochondroma; E) Faint staining of collagen type II in the cartilage cap in DEH F) Collagen type II staining around clusters of chondrocytes in DEH; G) Negative control collagen type II; (Magnification: A 2,5x, B-E 5x, F-G 20x)

\section{Collagen type $\mathrm{X}$}

In order to determine the presence of hypertrophic chondrocytes, sections were stained with an anti-collagen type $\mathrm{X}$ antibody. Both osteochondromas as DEH showed expression of collagen type $X$, while no signal was detected in negative controls. Expression of collagen type $X$ was observed in the extracellular matrix surrounding hypertrophic chondrocytes in osteochondromas (Figure 4A-B, white arrows). Expression of collagen type $\mathrm{X}$ was not detected in the cartilaginous matrix surrounding the proliferative chondrocytes, in the resting zone or in the trabecular bone of osteochondromas.

In addition, collagen type $\mathrm{X}$ was not detected in the entire cartilage cap (not shown) and clusters of chondrocytes in DEH (Figure 4C). 

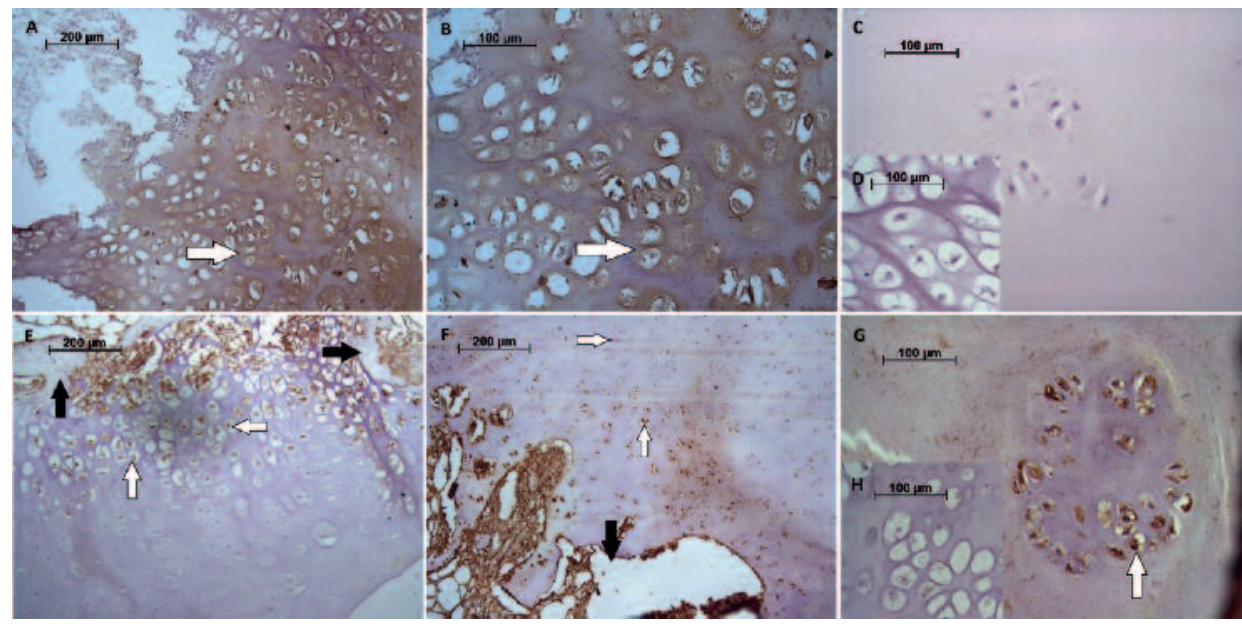

Figure 4. A-D Collagen type $X$ staining in osteochondroma and DEH, E-H Sox9 staining in osteochondroma and DEH; A) Positive pericellular staining of collagen type $\mathrm{X}$ in osteochondroma; B) Expression of collagen type $X$ in the extracellular matrix surrounding chondrocytes in osteochondroma; C) Absence of collagen type X expression in DEH; D) Negative control collagen type X; E) Positive nuclear expression of Sox9 in chondrocytes in osteochondroma; F)Expression of Sox9 in nuclei of chondrocytes in the cartilage cap in DEH; G) Expression of Sox9 in nuclei of a cluster of chondrocytes in DEH; H) Negative control Sox9. (Magnification, A, E,F 10x; B,C,D,G,H 20x)

\section{Sox 9}

Expression of Sox9, expressed in proliferative chondrocytes, was observed in both osteochondromas and DEH (Figure 4D-F). No signal was detected in negative controls. The nuclei of nearly all hypertrophic chondrocytes in osteochondromas in the vicinity of the chondro-osseous junction stained positive (Figure 4D, white arrows). In addition, a large amount of unresorbed calcified cartilage was observed (black arrows). Only a few proliferative chondrocytes showed expression for Sox9 (not shown).

Nuclear expression of Sox9 was detected in chondrocytes in the entire cartilage cap in DEH (Figure 4E, white arrows). In addition, cells in the underlying bone marrow beneath the zone of endochondral ossification clearly expressed Sox9. The characteristic chondrocyte clumps in DEH also expressed Sox9 in the nucleus (Figure 


\section{Chapter 6}

$4 \mathrm{~F}$, white arrow). The difference in amount of unresorbed calcified cartilage between osteochondromas and DEH is also visible, with large amounts in osteochondromas and small amounts in DEH (Figure 4D and E). No signal was detected in negative controls (Fig $4 \mathrm{H}$ ).

\section{Discussion}

We reported a histological evaluation of the extremely rare disorder $\mathrm{DEH}$ and histologically compared this disease with more common osteochondromas in $\mathrm{HMO}$, since lesions in both diseases are often stated as histologically identical ${ }^{14-22}$. Therefore the aim of this study was to test the hypothesis that DEH and osteochondromas are histologically identical diseases.

Instead of finding similarities, some major (immuno)histological differences between DEH and osteochondromas were shown between histological sections of both diseases. Osteochondromas showed a lobulated cartilaginous architecture with characteristics of the growth plate, i.e. different zones of maturations of chondrocytes. Compared with a normal growth plate, chondrocytes were less well aligned in columns. This finding is in line with previously reported studies of osteochondromas ${ }^{11,24-27}$. This partial growth plate structure was absent in the cartilage caps of our cases with DEH, where a thick, disorganized cartilage cap was observed.

Previous studies showed some characteristic morphological features of cartilage in DEH. These characteristics are clusters of chondrocytes surrounded by a faint fibrillar matrix ${ }^{7}$ and ossification centres in the cartilaginous matrix with a small area of unresorbed calcified cartilage above $i^{24,25,28,29}$. We identified these clusters of chondrocytes in both cases of DEH. These cell clusters were not observed in osteochondromas. The faint fibrillar matrix surrounding these clumps of chondrocytes was clearly detected in the Safranin-O and collagen type II staining. In addition, small areas of unresorbed calcified cartilage were present above the centres of ossification in the DEH samples. This observation in DEH is consistent with the literature and shows some significant morphological differences with osteochondromas. 
The performed immunohistological stainings revealed some additional histological differences between both diseases. In line with a previous study of Perl et al. who investigated $\mathrm{DEH}$, clear staining of collagen type II in the cartilage matrix surrounding the clumps of chondrocytes was detected ${ }^{29}$. This staining pattern deviates from the observations seen in osteochondromas, where the most obvious staining is detected in the extracellular matrix between the proliferating and hypertrophic chondrocytes, as expected based on a previous study in osteochondromas ${ }^{26}$. This finding suggests a difference in tissue characteristics between both diseases.

The performed immunostaining for collagen type $X$, a marker of hypertrophic chondrocytes, was not able to detect collagen type $\mathrm{X}$ in DEH. Besides, cells with the cellular morphological hypertrophic appearance were not identified in the sections of DEH. The results of this study, together with the results of Perl et al., who were also not able to identify collagen type $X$ and hypertrophic chondrocytes in $\mathrm{DEH}^{29}$, refute Trevor's hypothesis. Trevor hypothesized in his original article that DEH results from failure of hypertrophic chondrocytes to undergo apoptosis and therefore persist ${ }^{2}$. In contrast to DEH, collagen type $X$ was detected in the pericellular matrix between hypertrophic chondrocytes in osteochondromas as expected ${ }^{26,27,30}$. This difference in collagen type $\mathrm{X}$ expression also supports that both diseases are not identical.

Another hypothesis concerning the pathogenesis is that DEH results from a defect in keeping resident progenitor cells in a quiescent stage. This leads to accumulation of cell clusters and results in chondrocytes with phenotypic characteristics of chondroprogenitor as well as growth plate-like cells ${ }^{29}$. As a result, chondrocytes should be able to proliferate and express proliferative markers. Expression of the chondrogenic transcription factor Sox9, which is expressed in proliferative chondrocytes in normal growth plates, was therefore evaluated. Clear expression of Sox9 was observed in the nuclei of the chondrocytes arranged in clusters in DEH. These results may therefore support this newer hypothesis of the pathogenesis of DEH. According to our own hypothesis, in which we stated that DEH and osteochondroma were histologically identical, an unexpected result was observed. Differences in expression patterns of Sox9 were detected between DEH 


\section{Chapter 6}

and osteochondroma since Sox9 was expressed in some proliferative chondrocytes and almost all hypertrophic chondrocytes in osteochondromas. This was an unexpected result, since nuclear staining of the proliferative chondrocytes was expected. However, previous histological studies in osteochondromas also reported positive staining of proliferative markers in hypertrophic chondrocytes instead of proliferative chondrocytes ${ }^{30}$. It was suggested that osteochondroma chondrocytes presents some characteristics of hypertrophic cells (i.e. expression of collagen type $\mathrm{X})$, but these cells also have the ability to proliferate and fail to terminally differentiate ${ }^{30}$. The results of this study match with this suggestion, since hypertrophic chondrocytes were observed expressing both collagen type $\mathrm{X}$ and Sox9 in osteochondromas. However, the difference in expression of Sox9 in DEH and osteochondromas further strengthens the evidence that these diseases are not identical.

Thus, DEH and osteochondromas seem unidentical diseases based on their histological appearance, based on both morphological and immunohistological evaluation of sections of patients with DEH and osteochondromas. Furthermore, there are other differences between both diseases that imply a different aetiology. Both diseases appear at a different location and at a different age, with DEH arising from epiphyses of (young) children between 2 and 8 years of age 4 . In contrast, osteochondromas arise from the metaphysis of long bones and generally affect older children since osteochondromas develop and increase in size in the first and second decade of life and cease to grow when the growth plates close during puberty ${ }^{10}$. Next, exostosin (EXT)-related pathways are involved in the pathogenesis of osteochondromas and mutated EXT-genes are inherited by the patient in an autosomal dominant way in HMO. These EXT-genes are not involved in the pathogenesis of DEH as shown by Bovee et al. and until now, there is no evidence for genetic inheritance of $\mathrm{DEH} 7,10,11,24$. Finally, malignant transformation of osteochondromas to secondary peripheral chondrosarcoma is observed in 0.5-5\% of the patients with osteochondromas, while malignant transformation of DEH has not been reported before ${ }^{10,11}$. 


\section{Conclusion}

The hypothesis that DEH and osteochondromas are histologically identical diseases was examined in this study. Therefore, two cases with DEH were reported and compared histologically with two age and gender matched patients with osteochondromas as a result of $\mathrm{HMO}$. Since major morphological and immunohistological differences were detected between DEH and osteochondromas, we tend to reject this hypothesis and state that DEH and osteochondromas are different diseases based on histological appearance. These results, together with the other previously described clinical and molecular differences, suggest that DEH has a different aetiology than osteochondromas. However, additional studies using other techniques than immunohistochemistry are necessary to strengthen this evidence and to further elucidate the pathogenesis of DEH.

\section{Acknowledgements}

The authors would like to thank A. Cremers and T. Castermans for their help processing the samples in this study. No funding was received for this study. 


\section{Chapter 6}

\section{REFERENCES}

1. A. Mouchet JB. La tarsomégalie. J Radiol Electrol. 1926;10:289-93.

2. Trevor D. Tarso-epiphysial aclasis; a congenital error of epiphysial development. The Journal of bone and joint surgery British volume. 1950 May;32-B(2):204-13.

3. Fairbank TJ. Dysplasia epiphysialis hemimelica (tarso-ephiphysial aclasis). The Journal of bone and joint surgery British volume. 1956 Feb;38-B(1):237-57.

4. Arealis G, Nikolaou VS, Lacon A, et al. Trevor's Disease: A Literature Review regarding Classification, Treatment, and Prognosis apropos of a Case. Case reports in orthopedics. 2014;2014:940360.

5. Azouz EM, Slomic AM, Marton D, et al. The variable manifestations of dysplasia epiphysealis hemimelica. Pediatric radiology. 1985;15(1):44-9.

6. Wenger DR, Adamczyk MJ. Evaluation, imaging, histology and operative treatment for dysplasia epiphysealis hemimelica (Trevor disease) of the acetabulum: a case report and review. The lowa orthopaedic journal. 2005;25:60-5.

7. Bovee JV, Hameetman L, Kroon HM, et al. EXT-related pathways are not involved in the pathogenesis of dysplasia epiphysealis hemimelica and metachondromatosis. The Journal of pathology. 2006 Jul;209(3):411-9.

8. Goud AL, de Lange J, Scholtes VA, et al. Pain, physical and social functioning, and quality of life in individuals with multiple hereditary exostoses in The Netherlands: a national cohort study. The Journal of bone and joint surgery American volume. 2012 Jun 6;94(11):1013-20.

9. Wicklund $\mathrm{CL}$, Pauli RM, Johnston D, et al. Natural history study of hereditary multiple exostoses. American journal of medical genetics. 1995 Jan 2;55(1):43-6.

10. Bovee JV. Multiple osteochondromas. Orphanet journal of rare diseases. 2008;3:3.

11. Staal HM WA, Emans PJ, Ham JSJ, et al. Current knowledge on exostoses formation in hereditary multiple exostoses: Where do exostoses originate and in what way is their growth regulated? Hereditary Genetics. 2014 August 2014;3(3).

12. Skaggs DL, Moon CN, Kay RM, et al. Dysplasia epiphysealis hemimelica of the acetabulum. A report of two cases. The Journal of bone and joint surgery American volume. 2000 Mar;82(3):409-14.

13. Schmale GA, Conrad EU, 3rd, Raskind WH. The natural history of hereditary multiple exostoses. The Journal of bone and joint surgery American volume. 1994 Jul;76(7):986-92. 


\section{Dysplasia Epiphysealis Hemimelica}

14. Ouyang Z, Xu M, Li X, et al. Dysplasia epiphysealis hemimelica with involvement of the distal tibial epiphysis and talus: recurrence of a case and literature review. The Journal of foot and ankle surgery : official publication of the American College of Foot and Ankle Surgeons. 2014 Mar-Apr;53(2):199-202.

15. Wiart E, Budzik JF, Fron D, et al. Bilateral dysplasia epiphysealis hemimelica of the talus associated with a lower leg intramuscular cartilaginous mass. Pediatric radiology. 2012 Apr;42(4):503-7.

16. Takeuchi $\mathrm{H}$, Ito $\mathrm{K}$, Ogino $\mathrm{T}$, et al. A case of osteocartilaginous mass involving the coronoid process of the ulna: solitary osteochondroma or dysplasia epiphysealis hemimelica? Journal of shoulder and elbow surgery / American Shoulder and Elbow Surgeons [et al]. 2003 SepOct;12(5):510-3.

17. Vogel T, Skuban T, Kirchhoff C, et al. Dysplasia epiphysealis hemimelica of the distal ulna: a case report and review of the literature. European journal of medical research. 2009 Jun 18;14(6):272-6.

18. Vanhoenacker F, Morlion J, De Schepper AM, et al. Dysplasia epiphysealis hemimelica of the scaphoid bone. European radiology. 1999;9(5):915-7.

19. Shinozaki T, Ohfuchi T, Watanabe H, et al. Dysplasia epiphysealis hemimelica of the proximal tibia showing epiphyseal osteochondroma in an adult. Clinical imaging. 1999 May-Jun;23(3):168-71.

20. Takagi M, Kiyoshige $\mathrm{Y}$, Ishikawa A, et al. Multiple occurrence of osteochondromas in dysplasia epiphysealis hemimelica. Archives of orthopaedic and trauma surgery. 2000;120(5-6):358-60.

21. Azzoni R. Dysplasia epiphysealis hemimelica of the talus. Journal of orthopaedics and traumatology : official journal of the Italian Society of Orthopaedics and Traumatology. 2009 Mar;10(1):43-6.

22. Bosch C, Assi C, Louahem D, et al. Diagnosis and surgical treatment of dysplasia epiphysealis hemimelica. A report of nine cases. Orthopaedics \& traumatology, surgery \& research : OTSR. 2014 Dec;100(8):941-6.

23. Caron MM, Emans PJ, Cremers A, et al. Hypertrophic differentiation during chondrogenic differentiation of progenitor cells is stimulated by BMP-2 but suppressed by BMP-7. Osteoarthritis and cartilage / OARS, Osteoarthritis Research Society. 2013 Apr;21(4):604-13. 


\section{Chapter 6}

24. Glick R, Khaldi L, Ptaszynski K, et al. Dysplasia epiphysealis hemimelica (Trevor disease): a rare developmental disorder of bone mimicking osteochondroma of long bones. Human pathology. 2007 Aug;38(8):1265-72.

25. Karam AR, Birjawi GA, Saghieh S, et al. Generalized dysplasia epiphysealis hemimelica with contralateral sacro-iliac joint involvement. Skeletal radiology. 2008 Dec;37(12):1147-52.

26. Huch K, Mordstein V, Stove J, et al. Expression of collagen type I, II, X and Ki-67 in osteochondroma compared to human growth plate cartilage. European journal of histochemistry : EJH. 2002;46(3):249-58.

27. Legeai-Mallet L, Rossi A, Benoist-Lasselin C, et al. EXT 1 gene mutation induces chondrocyte cytoskeletal abnormalities and defective collagen expression in the exostoses. Journal of bone and mineral research : the official journal of the American Society for Bone and Mineral Research. 2000 Aug;15(8):1489-500.

28. Golles A, Stolz P, Freyschmidt J, et al. Trevor's disease (dysplasia epiphysealis hemimelica) located at the hand: case report and review of the literature. European journal of radiology. 2011 Feb;77(2):245-8.

29. Perl M, Brenner RE, Lippacher S, et al. Dysplasia epiphysealis hemimelica: a case report with novel pathophysiologic aspects. Clinical orthopaedics and related research. 2009 Sep;467(9):2472-8.

30. Benoist-Lasselin C, de Margerie E, Gibbs L, et al. Defective chondrocyte proliferation and differentiation in osteochondromas of MHE patients. Bone. 2006 Jul;39(1):17-26. 
Dysplasia Epiphysealis Hemimelica 


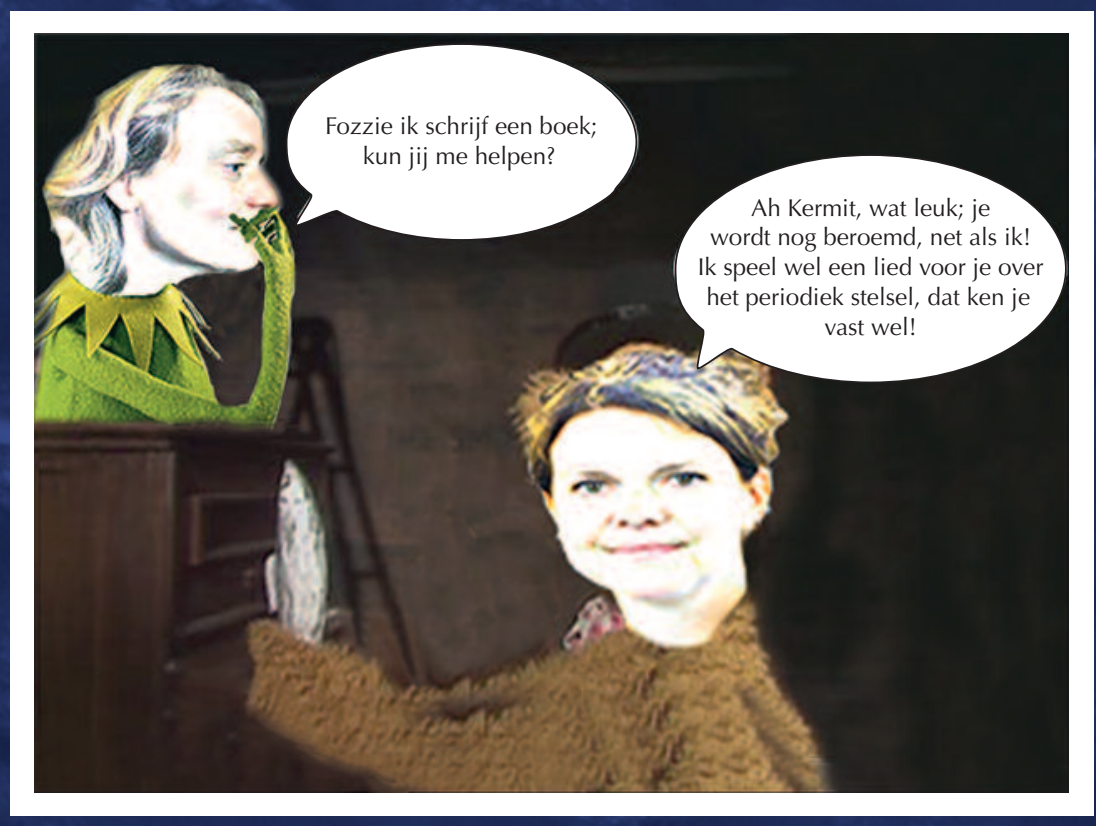




\section{ChAPTER SEVEN}

\section{The use of Whole-Body MR Imaging in children with $\mathrm{HMO}$, an extended case study in two patients}

Heleen M. Staal, Marjolein HG. Dremmen, Simon GF. Robben, M. Adhiambo Witlox, Lodewijk W. van Rhijn. 


\section{Chapter 7}

\section{AbStRACT}

Background: Patients with Hereditary Multiple Osteochondromas (HMO) undergo frequent radiographs to evaluate the growth of their osteochondromas. The conventional radiographic images clearly show the growth of the bony part of the osteochondromas and the growth direction of the long bones. The radiographs do not show the cartilage cap on top of the osteochondroma nor do they show the surrounding soft tissue or the cartilage of the nearby epiphysis. Alongside these disadvantages, taking frequent radiographs carries the potential risk of inducing malignant degeneration through ionizing radiation. In this study we investigated the use of whole-body MR imaging as a screening tool to follow patients with HMO.

Findings: Two HMO affected children underwent two whole-body MR imaging scans in one year's time to identify the osteochondromas and to evaluate their growth. The MR images were compared to regular follow-up radiographs of these patients. All radiographically detectable osteochondromas were visible on the whole-body MR images. At least one osteochondroma was clearly seen on the whole-body MR images before detection was possible on the radiographs. The proton density sequence with fat suppression proved to be the best sequence to visualize osteochondromas.

Conclusion: Whole-body MR imaging is an effective follow-up tool for patients with Hereditary Multiple Osteochondromas. 


\section{INTRODUCTION}

Hereditary Multiple Osteochondromas or exostoses (HMO or HME) is an autosomal dominant inherited disease caused by mutated exostosin genes. HMO is characterized by the outward growth of cartilage-capped bone tumours called osteochondromas. The osteochondromas grow on the external surface of bones and contain a bone marrow cavity continuous with the normal bone cavity ${ }^{1,2}$. They often occur at the metaphysis of the long bones, but are also found on the spine, the scapulae, the ribs and the pelvis. The osteochondromas develop in the first decade of life and continue to grow until the patient reaches skeletal maturity. Most osteochondromas are symptomless, but patients can suffer from pain and discomfort of the osteochondromas giving pressure on the overlying structures such as tendons, ligaments, nerves and even on the spinal cord. HMO can further lead to growth disturbances including Madelung's deformity (40-60\%), unequal limb length (10$50 \%)$, joint deformity (2-55\%) and a disproportionally short stature (37-45\%)

In patients diagnosed with $\mathrm{HMO}$ multiple conventional radiographs are taken for follow-up. The radiographs give clear information about the bones and the ossified osteochondromas. Furthermore, growth deformations of the long bones can be detected. In adults regular follow-up every (other) year is advocated ${ }^{7}$. In fast growing children every six months radiographs are taken to evaluate the growth deformities and to determine the need for early intervention. For example, early resection of the osteochondromas in the forearm can prevent forearm deformity and early hemi-epiphyseal stapling can prevent ankle deformities. Since growth deformities and joint dislocations in children with $\mathrm{HMO}$ can cause significant disability; early treatment of the deformity may prevent or decrease later deterioration of function. The aim of treatment is surgical resection of the masses the and prevention of deformities ${ }^{8-10}$.

Next to the deformities osteochondromas can cause pain. Pressure of the osteochondroma on the surrounding soft tissues can be painful. The top of the osteochondroma, the cartilage cap, is not ossified and therefore cannot be seen on the radiographs. These soft tissues are not seen on radiographs but are clearly visible on MR images. MR images can also provide information about the cartilage of the nearby epiphysis and thus about the potential growth deformation. 


\section{Chapter 7}

Whole-body MR imaging ( $\mathrm{Wb} \mathrm{MRI}$ ) is a non-invasive screening technique that acquires images of the entire body. Patients with $\mathrm{HMO}$ in regular follow-up ${ }^{7}$ may need a high number of radiographs (estimated 10-20 radiographs per year in a fast growing child) and thereby receive a significant dose of ionizing radiation with the potential risk of inducing malignant degeneration of the cartilage cap of the osteochondroma. Because of the visibility of the top and of the tissues surrounding the osteochondromas and the visibility of the epiphysis of the long bones, and at the same time the lack of radiation, Wb MRI is a promising imaging modality for serial imaging surveillance in the paediatric age group.

The advantages of MR imaging over radiographic follow up are known, but until now it was difficult to scan the whole body in a reasonable scanning time. To make a whole body scan every body part had to be scanned separately and sometimes in different coils. Since modern MRI technology provides MRI systems with high-density coil elements with flexible switching possibilities, multiple receiver channels and automatic table movements, Wb MRI has become clinically available. The use of Wb MRI in the paediatric population for oncologic applications has become more established in recent literature ${ }^{11-17}$. The role for Wb MRI in other multisystem disease processes still has to be investigated. As mentioned by Lenk in 2004 the Wb MRI has a potential application in the regular screening of children with $\mathrm{HMO}$ as alternative for screening with radiographs ${ }^{18}$.

The aim of this study was to qualitatively evaluate the use of the Wb MR imaging in $\mathrm{HMO}$ affected children. Therefore the regular conventional radiographs of two $\mathrm{HMO}$ affected children were compared to the Wb MRI findings during one year. The images in both methods were evaluated to see if all osteochondromas could be identified and to see if the possible deformity of the long bones was equally visible. Furthermore, we analysed the Wb MR images to verify if early detection of osteochondromas was possible.

\section{Patients AND methods}

Two patients were included. Both patients were diagnosed with $\mathrm{HMO}$. The evaluated imaging studies consisted of four consecutive whole-body MR imaging studies in the period from 2012 to 2014. 
Patient one was a two-year-old boy who was seen at the orthopaedic outpatient clinic because of palpable swelling on the thorax and the scapulae. His mother was diagnosed with $\mathrm{HMO}$. Conventional radiographs showed multiple osteochondromas of the pelvis, multiple ribs, the scapulae, and the long bones of the upper and lower extremities. His right ulna showed severe growth deformity and his right tibia minor growth deformity. Learning about the technique of Wb MRI and the possible use of the technique in patients with $\mathrm{HMO}^{17,18}$, we considered this patient suitable. At the age of three years we performed a Wb MRI. The first scan was performed without anaesthesia or sedatives. Mother asked for general anaesthesia for the second scan because her son had been anxious during the first scan. The second scan was done one year later under general anaesthesia.

The second patient was a seven-year-old boy diagnosed for several years with $\mathrm{HMO}$. His father was also diagnosed with the disease. Both Wb MRI scans were done with a one-year interval without general anaesthesia.

In both patients every six months a regular check-up was done in a fast growing period, with conventional radiographs and after one year the Wb MRI was repeated. The study was performed in accordance with the Declaration of Helsinki. Both the patients and their parents gave their informed consent prior to their inclusion.

\section{Imaging Technique}

MR imaging was performed with a 1.5T MRI scanner (Ingenia; Philips Healthcare, Best, the Netherlands). Ingenia's Smart Selection automatically selects the coils and coil elements which contribute to the highest signal-to-noise ratio in any region or any length of the field of view. As there is no consensus about which combination of MR sequences provides the highest diagnostic accuracy combined with reasonable time efficiency, at the beginning of the study a combination of sequences for screening for osteochondroma was chosen based on limited experience. The proposed imaging protocol consisted of a 1-mm-thick balanced Steady State Free Precession (bSSFP) sequence in the coronal plane (TR, $17 \mathrm{~ms}$; TE, shortest ms; matrix, 432 × 432; FOV, $430 \times 326$ × 150 mm; 150 sections; acquisition time, 7 minutes), a 1-mm-thick 3D Volume Isotropic Turbo-Spin-Echo T1-weighted sequence in the coronal plane (TR, 350 ms; TE, 13 ms; matrix, 432 x 432; FOV, $430 \times 322.5$ x 150 mm; 150 sections; mean acquisition time, 6 minutes 25 seconds) and a 5-mm-thick 


\section{Chapter 7}

multi-turbo spin-echo Proton-Density (PD)-weighted Spectral Presaturation with Inversion Recovery (SPIR) sequence in the coronal plane (TR, 1800-4000 ms; TE, 20 ms; matrix, $512 \times 512$; FOV, $220 \times 220 \times 275$ mm; 50 sections; mean acquisition time, 8 minutes 5 seconds).

\section{Imaging Evaluation}

The MR images were evaluated by experienced paediatric radiologists. The diagnosis of osteochondroma on Wb MRI was based on the presence of cartilage-capped osseous excrescence (sessile or pedunculated) with continuous cortex and marrow extending from underlying bone and pointing away from the epiphysis. The osseous excrescence had to be detectable on at least one of the different sequences of the Wb MRI protocol. The Wb MRI findings were correlated with radiographs. At the time we started performing Wb MRI studies no protocols were available, nor standard detection protocols for paediatric patients.

The visibility of the osteochondromas on the Wb MRI was compared to the visibility on the conventional radiographs. To detect growth deformations, the angle between the axis of the long bone and the epiphysis or articulation was calculated to determine the valgus and varus malalignment and ante- or recurvation.

\section{ResUlts}

In total 64 osteochondromas were found on the conventional radiographs of the long bones of the two patients. All osteochondromas that were detectable on conventional radiographs could also be identified on the Wb MR images, even though one of the osteochondromas, situated on the left ulna of the first patient, was very clear on the radiographic images, but was hard to identify on MRI. This osteochondroma was located on the left distal ulna on the dorsal side. It is likely that this osteochondroma would have been missed if the first screening had been done by Wb MRI alone. However the visibility was better on the second scan.

The size and precise location of at least 6 osteochondromas in the second patient, were much easier to evaluate on the Wb MR images. By using the Wb MR images it was possible to distinguish several osteochondromas that were located close to one 
another. These osteochondromas were located so close to one another that on the radiographs they projected like one big osteochondroma, but on the Wb MRI the osteochondromas were visible separately and located more ventral and more distal to each other instead of in continuity. Furthermore, in this patient it was possible to distinguish several osteochondromas from the normal bony outgrowth of the trochanter on the $\mathrm{Wb}$ MRI. On the conventional radiographs these osteochondromas at the proximal femur were easily mistaken for the major trochanter. Five osteochondromas were seen on the Wb MR images but could not be identified on the conventional radiographs. This was due to their location of which no proper radiographic images were available. One osteochondroma in the first patient was visible on the Wb MRI before it became apparent on the conventional radiographs. It was situated on the right distal fibula near the tibia. It was visible on the first as well as on the second Wb MRI scan. On the first MRI scan only a small bump of osteochondromal cartilage was detectable, the onset of an osteochondroma, on the lateral site of the distal tibia and the medial site of the distal fibula, indicated in figure $1 \mathrm{~b}$ with a white arrow (figure 1).

Of all osteochondromas detected on the Wb MR images, the cartilage cap could be clearly identified. In the same patient the diameter of the cartilage cap varied widely among the different osteochondromas and was not related to the volume of the bony outgrowth. The influence on the surrounding soft tissue structures of the osteochondroma was clearly visible, especially in the thoracic osteochondromas. No impingement or bursa formation was detected.

Measuring the angles between the axis of the long bone and the epiphysis or articulation to detect growths deformations, was equally possible on the radiographs as well as on the Wb MR images. In both imaging techniques the articulation or epiphysis is shown along with the axis of the long bone, making it possible to detect axial deformities with both techniques. On top of the detection of the deformity, the Wb MR images showed the cartilage of the epiphyseal plate. No epiphyseal damage was seen even though a clear shortening of the right ulna was detected in the first patient, the two-year-old.

No signs of malignant degeneration of the osteochondromas were found.

On average 20 conventional radiographs of the skeleton were taken per patient per year, respectively 18 in the first and 22 in the second patient. For this radiographic screening the estimated total radiation dose is $1,8 \mathrm{mSv}$ per patient per year. 


\section{Chapter 7}

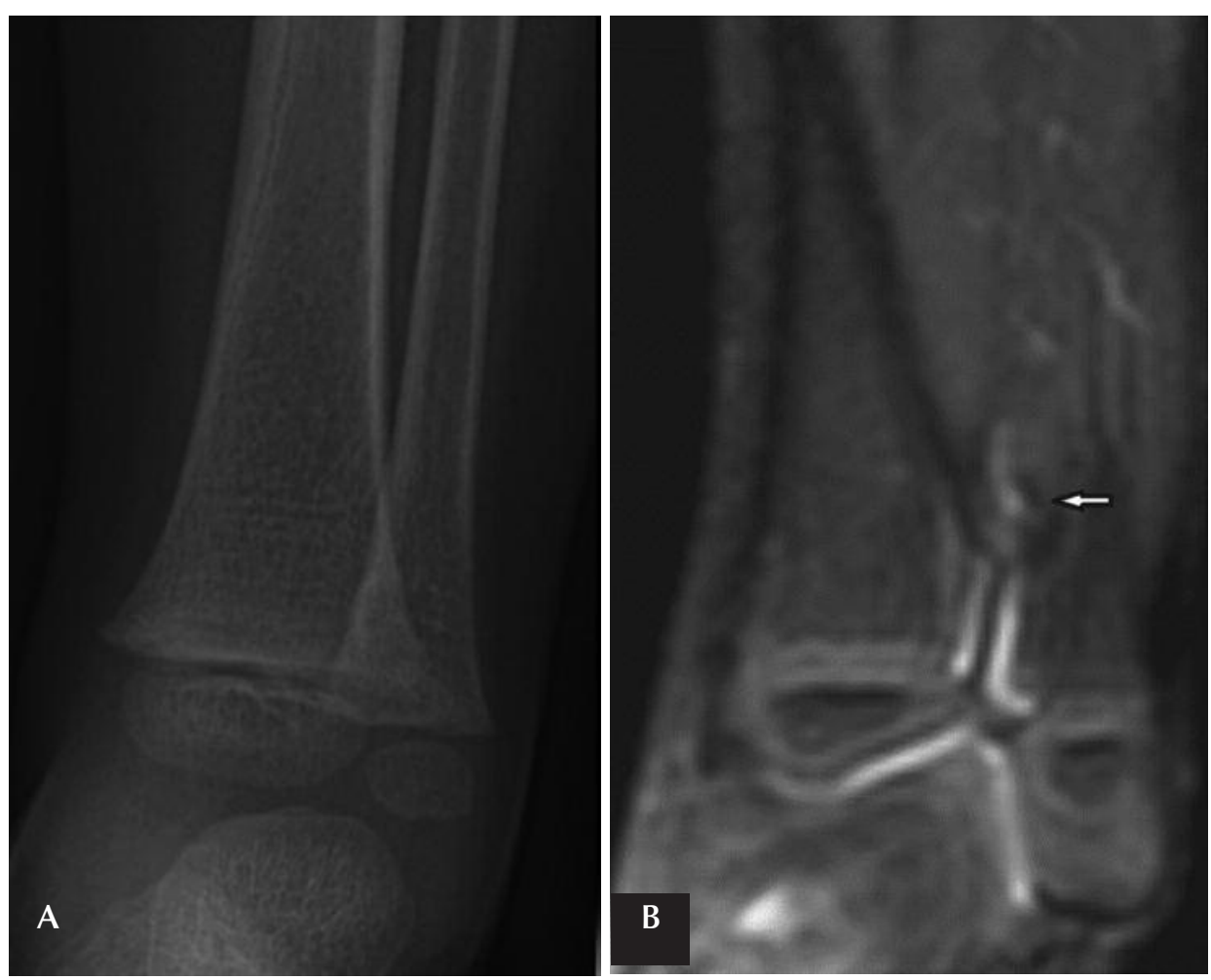

Figure 1: early detection of an osteochondroma. Figure $1 \mathrm{~A}$, conventional radiograph of the distal tibia and fibula in AP view, showing no abnormalities. Figure 1B, coronal fat-suppressed proton density weighted MR image of the distal tibia en fibula showing a cartilage bump (white arrow).

\section{Discussion}

In this two-patient case study Whole body MR images show more accurate detectability of the osteochondromas compared to conventional radiographs. Especially in the young children the osteochondromas are more cartilaginous and therefore better visualized on the MR images. These young children would probably benefit more from the $\mathrm{Wb} M \mathrm{MI}$ as a screening device. This would also imply less ionizing radiation for screening purposes in the lifespan of these children. The number of radiographs taken in one year was on average 20 , which approximately doubled 

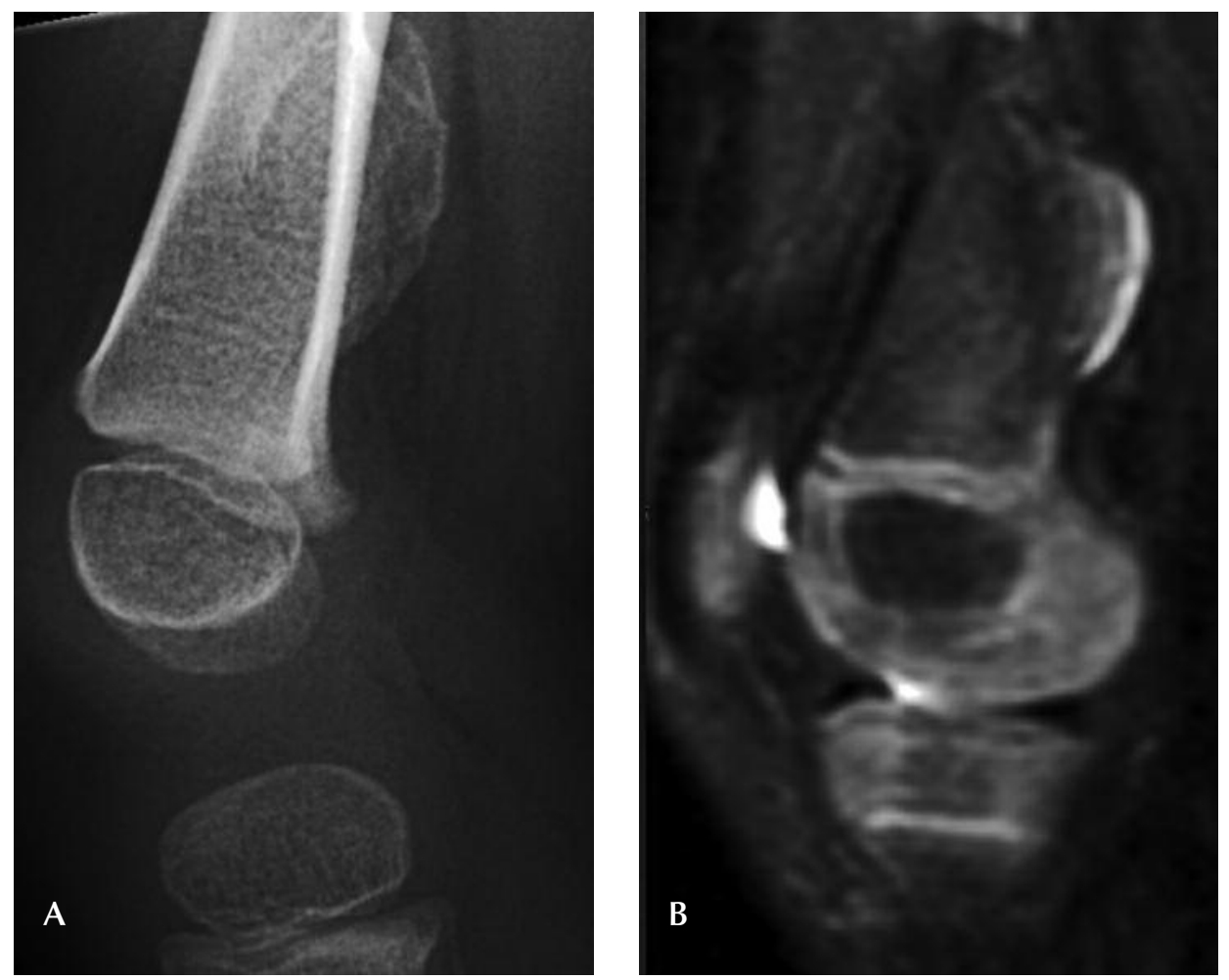

Figure 2: visibility of the cartilage of the osteochondroma. Figure 2A, lateral conventional radiograph of the knee with a sessile osteochondroma on the dorsal site of the distal femur. Figure 2B, sagittal Wb MR image, proton density sequence of the knee mark the clearly visible cartilage cap of the osteochondroma lighting up more clearly than the articular cartilage of the knee.

their effective year dose from natural background radiation. Using the Wb MRI for screening purposes would sharply decrease their exposure to ionizing radiation.

The problems that were encountered during the scanning and evaluation are described. The scanning time of the Wb MRI scans compared to obtaining the radiographs was longer. Besides the time, the fact that the children had to lie still in a limited space was a disadvantage of the Wb MRI scan. Several scans were not suitable for evaluation due to motion artefacts. To reduce the motion artefacts and because of anxiety, the first patient needed to be anesthetized during the second 


\section{Chapter 7}

scan. This makes the Wb MRI less attractive for serial imaging surveillance in children under the age of four.

The variable rotation angles of the position of fore example the forearm during scanning made it hard to compare the MR images to the conventional radiographs. This variation also made it difficult to compare the consecutive scans. A more standardized positioning during scanning could prevent this problem.

Since there is no standard scanning protocol, the detectability of the osteochondroma itself was distinct among the different scan protocols. For example on the 3D TSE T1 and the bSSFP sequences, it was more difficult to distinguish the cartilage cap of the osteochondroma from the surrounding soft tissue. On the PD SPIR sequence the cartilage of the osteochondroma was more hyper intense compared to functional cartilage. Hypothetically this is due to the higher water concentration in the osteochondromal cartilage caps compared to the functional cartilage, possibly due to the lack of pressure on the osteochondromal cartilage (figure 2). Since the osteochondromas were best visualized on the PD SPIR sequence, we would consider this sequence the most suitable sequence for screening purposes in $\mathrm{HMO}$ patients.

In this study we did not expect to find malignant degeneration because the patients were young, malignant transformation before the age of 20 is distinctly unusual $^{19}$. In adult HMO patients the Wb MRI screening might be beneficial in this respect. So far bone-scintigraphy and FDG-PET scans have been applied for early detection of malignant transformation in adults ${ }^{20-22}$.

Searching for newly formed osteochondromas, the MR images showed one small new osteochondroma. It was found on the distal fibula and was not detectable on the conventional radiographs. No lose cartilage islands were detected. Douis et al. could, not confirm the widely believed theory of an osteochondroma arising from misplaced cartilage in the metaphysis or an extension from the growth plate into the metaphysis, in a MRI study in 2012, nor could this case study confirm this hypothesis ${ }^{23}$. The imaging of very small osteochondromas, however, can potentially shed a light on the place of origin of the osteochondroma in the future and in the long run might lead to early treatment of the disease.

The costs of a Wb MRI are substantially higher than the costs of conventional radiographs, especially if general anesthesia has to be administered. For a standard 
Wb MRI the costs are about 400 euros compared to 50 euros for the conventional radiographs. No cost benefit analyses are available. Wuyts et al. suggested that monitoring the size of osteochondromas in adults may aid in early identification of malignant degeneration, but they also found no cost/benefit analyses to support this routine ${ }^{8}$.

In summary this case study shows that the use of Wb MRI is suitable as a screening tool for follow-up in $\mathrm{HMO}$ affected paediatric patients. Wb MRI showed accurate detectability of the osteochondromas, it reduces the exposure to ionising radiation and might lead to early detection of the osteochondromas. However, it is more time consuming and at a higher cost than the conventional radiographic imaging follow-up, especially when general anaesthesia is needed. There is need for a standardized protocol of the Wb MRI settings for screening purpose. In this case study the PD SPIR sequence was found to be the most suited sequence, taken in coronal and sagittal plane and with the limb in a standardized position. For future studies we recommend the use of Wb MRI screening for children that have no need for anaesthesia during the scan. With the use of the advised protocol the time needed for routine $\mathrm{Wb} M \mathrm{MRI}$ can be reduced.

\section{Conclusion}

Whole-body MRI is an effective screening tool in the follow-up of patients with Hereditary Multiple Osteochondromas. The osteochondromas can be accurately visualized. The PD SPIR sequence is the most suitable sequence for the detection of the osteochondromas and evaluation of their cartilage cap. The major disadvantage is the potential need for general anaesthesia to perform the scan in the very young age group.

\section{Acknowledgements}

We thank Brent van der Doelen, Maastricht University, for collecting the radiological and MRI data. 


\section{Chapter 7}

\section{REFERENCES}

1. Khurana, J. Bovee, J. Osteochondroma. World Health Organisation Classification of tumours. Pathology and Genetics of Tumours of Soft Tissue and Bone 2002, 234-236.

2. Bovee, J.; Hogendoorn, P.C. Multiple osteochondromas. World Health Organisation Classification of tumours. Pathology and Genetics of Tumours of Soft Tissue and Bone 2002, 260-362.

3. Hennekam, R.C. Hereditary multiple exostoses. J Med Genet 1991, 28, 262-266.

4. Legeai-Mallet, L.; Munnich, A.; Maroteaux, P.; Le Merrer, M. Incomplete penetrance and expressivity skewing in hereditary multiple exostoses. Clin Genet 1997, 52, 12-16.

5. Schmale, G.A.; Conrad, E.U., 3rd; Raskind, W.H. The natural history of hereditary multiple exostoses. J Bone Joint Surg Am 1994, 76, 986-992.

6. Wicklund, C.L.; Pauli, R.M.; Johnston, D.; Hecht, J.T. Natural history study of hereditary multiple exostoses. Am J Med Genet 1995, 55, 43-46.

7. Hameetman, L.; Bovee, J.V.; Taminiau, A.H.; Kroon, H.M.; Hogendoorn, P.C. Multiple osteochondromas: Clinicopathological and genetic spectrum and suggestions for clinical management. Hered Cancer Clin Pract 2004, 2, 161-173.

8. Wuyts, W.; Schmale, G.A.; Chansky, H.A.; Raskind, W.H. Hereditary multiple osteochondromas. In Pagon RA, A.M., Ardinger HH, et al, Ed. University of Washington, Seattle, 2000 Vol. GeneReviews®Internet.2000 Aug 3Updated 2013 Nov 21.

9. Saglik, Y.; Altay, M.; Unal, V.S.; Basarir, K.; Yildiz, Y. Manifestations and management of osteochondromas: A retrospective analysis of 382 patients. Acta Orthop Belg 2006, 72, 748-755.

10. Tompkins, M.; Eberson, C.; Ehrlich, M. Hemiepiphyseal stapling for ankle valgus in multiple hereditary exostoses. Am J Orthop (Belle Mead NJ) 2012, 41, E23-26.

11. Darge, K.; Jaramillo, D.; Siegel, M.J. Whole-body mri in children: Current status and future applications. Eur J Radiol 2008, 68, 289-298.

12. Tzaribachev, N.; Well, C.; Schedel, J.; Horger, M. Whole-body mri: A helpful diagnostic tool for juvenile dermatomyositis case report and review of the literature. Rheumatol Int 2009, 29, 1511-1514.

13. Schmidt, G.P.; Haug, A.; Reiser, M.F.; Rist, C.whole-body mri and fdg-pet/ct imaging diagnostics in oncology. Radiologe 2010, 50, 329-338. 
14. Goo, H.W. Regional and whole-body imaging in pediatric oncology. Pediatr Radiol 2011, 41 Suppl 1, S186-194.

15. Atkin, K.L.; Ditchfield, M.R. The role of whole-body mri in pediatric oncology. J Pediatr Hematol Oncol 2014, 36, 342-352.

16. Friedman, D.N.; Lis, E.; Sklar, C.A.; Oeffinger, K.C.; Reppucci, M.; Fleischut, M.H.; Francis, J.H.; Marr, B.; Abramson, D.H.; Dunkel, I.J. Whole-body magnetic resonance imaging (wbmri) as surveillance for subsequent malignancies in survivors of hereditary retinoblastoma: A pilot study. Pediatr Blood Cancer 2014, 61, 1440-1444.

17. Schmidt, G.P.; Reiser, M.F.; Baur-Melnyk, A. Whole-body imaging of the musculoskeletal system: The value of mr imaging. Skeletal Radiol 2007, 36, 1109-1119.

18. Lenk, S.; Fischer, S.; Kotter, I.; Claussen, C.D.; Schlemmer, H.P.possibilities of whole-body mri for investigating musculoskeletal diseases. Radiologe 2004, 44, 844-853.

19. Murphey, M.D.; Choi, J.J.; Kransdorf, M.J.; Flemming, D.J.; Gannon, F.H. Imaging of osteochondroma: Variants and complications with radiologic-pathologic correlation. Radiographics 2000, 20, 1407-1434.

20. Purandare, N.C.; Rangarajan, V.; Agarwal, M.; Sharma, A.R.; Shah, S.; Arora, A.; Parasar, D.S. Integrated pet/ct in evaluating sarcomatous transformation in osteochondromas. Clin Nucl Med 2009, 34, 350-354.

21. Kok, H.K.; Fitzgerald, L.; Campbell, N.; Lyburn, I.D.; Munk, P.L.; Buckley, O.; Torreggiani, W.C. Multimodality imaging features of hereditary multiple exostoses. Br J Radiol 2013, 86, 20130398.

22. Sanchez-Rodriguez, V.; Medina-Romero, F.; Gomez Rodriguez-Bethencourt, M.A.; Gonzalez Diaz, M.A.; Gonzalez Soto, M.J.; Alarco Hernandez, R. Value of the bone scintigraphy in multiple osteochrondromatosis with sarcomatous degeneration. Rev Esp Med Nucl Imagen Mol 2012, 31, 270-274.

23. Douis, H.; Davies, A.M.; James, S.L.; Kindblom, L.G.; Grimer, R.J.; Johnson, K.J. Can mr imaging challenge the commonly accepted theory of the pathogenesis of solitary enchondroma of long bone? Skeletal Radiol 2012, 41, 1537-1542. 

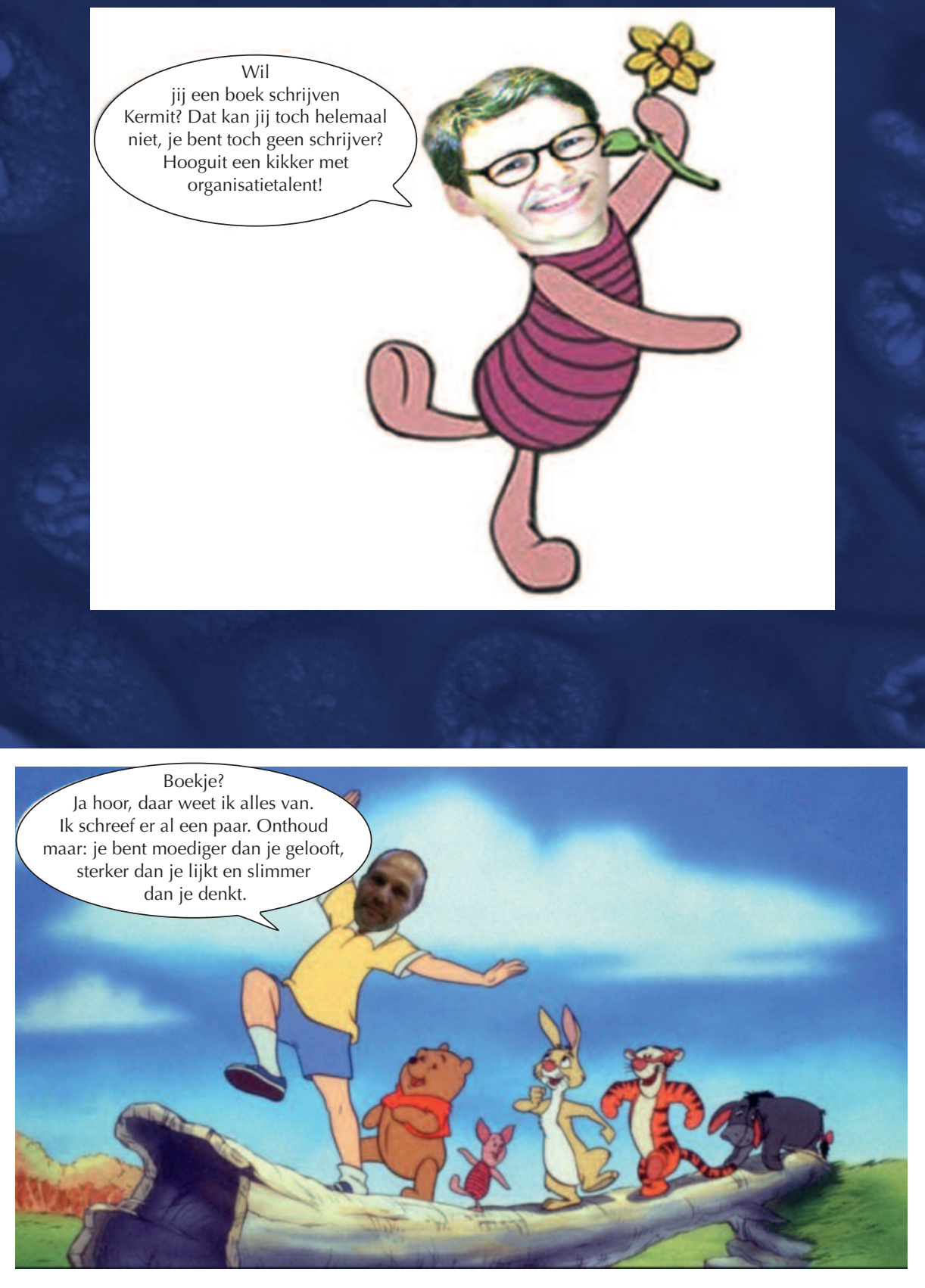


\section{CHAPTER EIGHT}

\section{Time-lapse technique used to uncover the naturel growth of osteochondromas of the wrist in patients with $\mathrm{HMO}$}

Heleen M. Staal, John Derwall, M. Adhiambo Witlox, Lodewijk W. van Rhijn 


\section{Chapter 8}

\section{AbStRACT}

In patients with Hereditary Multiple Osteochondromas ( $\mathrm{HMO}$ ) shortening and deformity of the long bones is a well-known sequel. Especially deformity at the wrist and ankle occur. The cause of the shortening or deformity is still unknown.

To reveal the development of the long bones and the influence of the osteochondromas on the growth we used time-lapse technique. Radiographic images, taken for clinical reasons, of six $\mathrm{HMO}$ affected patients with known osteochondromas on the distal end of the lower arm were selected and adapted to make them suitable for use with a time-lapse technique. The images were placed in chronological order. The ulnar shortening, lunate position and radial inclination were registered, as were the clinical problems. The hypothesis being that slow occurring developmental deformities are easier comprehended and qualitatively studied when the changes are accelerated.

Result; None of the patients had radial head dislocation at the elbow. The majority of the wrists had decreased ulnar length and increased radial inclination. The clinical problems were limited. The osteochondromatic growth did not seem related to the wrist growth, but when the inclination of the radius was steep osteochondromas were present in the distal radius and the ulna was shortened. Some osteochondromas disappeared.

Conclusion; Time-lapse videos of wrists of patients with $\mathrm{HMO}$ showed no relation between growth of wrists and osteochondromas but it did show osteochondromas disappearing. A steep radial inclination seems related to ulnar shortening and might be due to proximity of radial osteochondromas. Patients had marked radiological changes, but relatively few clinical problems. 


\section{INTRODUCTION}

Patients with Hereditary Multiple Osteochondromas (HMO) or Hereditary Multiple Exostosis suffer from an autosomal dominant inherited disease that causes osteochondroma formation. Osteochondromas are characterised by the outward growth of cartilage-capped bone tumors on the long bones; the bone marrow cavity is continuous with the normal bone cavity ${ }^{1,2}$. They are most often found on the metaphysis. Osteochondromas develop in the first decade of life and cease to grow until the patient reaches skeletal maturity. Patients with $\mathrm{HMO}$ can suffer from pain and discomfort when osteochondromas put pressure on the overlying structures such as tendons, nerves or even the spinal cord. Besides discomfort and pain in almost half the population, $\mathrm{HMO}$ leads to growth disturbances including Madelung-like deformity (40-60\%), unequal limb length (10-50\%), joint deformity (2-55\%) and a disproportionally short stature $(37-45 \%)^{3-6}$. The deformities slowly develop over time during skeletal growth.

About half of the patients develop wrist deformities resembling Madelungs deformity. Madelungs deformity was first described by Otto W. Madelung in 1878 as an epiphyseal growth plate disturbance characterized by dorsal and radial bowing of the radius ${ }^{7}$. In $\mathrm{HMO}$ patients a forearm pseudo-Madelungs deformity is described with shortening of the ulna and secondary bowing of the radius ${ }^{8}$. The deformity has cosmetic effects and decreases grip strength. Treatment remains controversial since the outcome is moderate ${ }^{9}$.

The aim of this study is to increase understanding of development of the wrist in $\mathrm{HMO}$ over time. To visualize the slow developing deformity the time-lapse technic is used. The hypothesis being that slow occurring developmental deformities are easier comprehended and qualitatively studied when the changes are viewed in accelerated sequence. 


\section{Chapter 8}

\section{Patients AND MEthod}

In general if growth is slow it is difficult to visualise. To facilitate the observer we can accelerate the growth of the viewed subject by taking images of the subject at regular times during growth and view them in an accelerate way just like we can, for example, observe a tree growing over 30 years time in a one minute timeframe. This technique is called time-lapse. Time-lapse is defined as "photographic technique of taking a sequence of frames at set intervals to record changes that take place slowly over time". This time-lapse technique was used on radiographs taken of 11 wrists of 6 patients diagnosed with $\mathrm{HMO}$ with at least one osteochondroma on the distal forearm. The anterior-posterior and lateral radiographs of the wrists were collected over a minimum of 5 years. The patients' ages varied between 7 and 15 .

All of the selected wrist images were identically scaled and then framed in a similar fashion. The grey scale was adjusted using adobe Photoshop. In the frames the anterior-posterior images were placed in a chronological order by using Keynote. To properly outline the images a fixed point was selected at the base of the osteochondroma nearest to the radial epiphysis. The view time per image was 3 seconds. In all images the ulnar shortening, lunate position and radial inclination were registered and shown in the last image. The clinical problems of the wrist were registered from the patient charts.

\section{RESULTS}

The videos of the wrists can be viewed using the QR-code. All of the forearms had distal osteochondromas, 3 on the radius alone, 1 on the ulna alone and 7 on both ulna and radius. Patient nr 10 underwent a resection of a wide osteochondroma. Of all 11 wrists, 9 had a shortening of the ulna. The inclination of the radius was increased in all patients (above 25 degrees) and over 30 degrees in 6 of the 11 wrists. The lunate was slipped over $50 \%$ in 3 of the 11 cases and between $25-50 \%$ in the remaining 8 . 


\section{Clinical problems}

None of the patients had a radial head dislocation at the elbow. Two patients had a marked ulnar drift and a decreased radial movement from 10 degrees. In one of these two patients the volar flexion was limited to 50 degrees. One patient had a limited supination of 70 degrees. One patient used a wrist brace for pain relief during repetitive activities.

Adapting the radiographs in this manner showed the growth of osteochondromas in relation to the growth of the wrists. In the ulnar length no clear relation could be found with the proximity of osteochondromas on the distal ulna. The radial deformity seemed more severe when osteochondromas were present in its proximity ( 5 out of 6 ) of the distal radius. It also seemed related to the shortening of the ulna (6 out of 6$)$.

Remarkably in the videos of patient nr 2 and patient nr 9, the osteochondromas on the ulnar side of the radius disappear, without surgical intervention.

\section{Discussion}

In the selected wrists the majority had shortening of the ulna and increased radial inclination. Only 3 out of the 11 wrists showed a carpal slip over 50\%. The deformity of the wrists in $\mathrm{HMO}$ develops slowly over time and can be visualised using the time-lapse technic. The study shows that some patients had a gradually shorting of the ulna, which was not directly depending on the growth of osteochondromas. The radial deformity seems more depended on the proximity of radial osteochondromas.

The described development of a Madelung-like deformity at the wrists of about half of the HMO affected patients could not completely be seen in the patients from this study ${ }^{10-12}$. The described shortening of the ulna however was clearly visible in the majority of the patients. In our patient group only one patient suffered from wrist pain and three patients had decreased wrist function. One patient was operated upon to resect a large radial osteochondroma oppressing the ulna. Even after resection this patient had a decreased volar flexion. The involvement of the upperlimb bones by HMO should be associated with greater loss of function because of 


\section{Chapter 8}

the paired bones as described by Abe et al., ${ }^{13}$ but many authors underline that deformities of the upper extremity in patients who have $\mathrm{HMO}$ are well tolerated and lead to little loss of function as seen in our study ${ }^{14-17}$.

The relationship between the ulnar length and the sliding of the lunate could not be found. However there seemed to be a relationship between the radial inclination and the ulnar shortening.

A study by Burgess et al. described no correlation between the radial articular angle and the ulnar position ${ }^{11}$. However, the study by Gottschalk et al. - who separated the different types of forearm deformity - found a correlation between the growth arrests in the ulna, caused by osteochondromas, that preceded the deformity seen in the distal radius in one subgroup ${ }^{10}$. Possibly in some types of growth disturbance the ulnar growth can influence the radial development or vice versa.

The most obvious deformity in the HMO wrists is the ulnar shortening. This automatically leads to a disruption of the distal radio-ulnar joint (DRUJ). Many others have described this problem ${ }^{12,18-20}$. Notwithstanding the joint deformity, the function of the DRUJ stays intact. However, the marked shortening is considered important in the planning of treatment for forearm deformities ${ }^{10-14,15,20-24}$. Some advocate early aggressive management to prevent deformity and disability $12,15,19-21,24-28$ and others are more conservative and have reported satisfactory function in skeletal mature patients without surgical intervention ${ }^{3,14}$ The natural history of wrist deformity in $\mathrm{HMO}$ remains unknown and the role and timing of surgical treatment therefore controversial. Perhaps monitoring the changes over time can help unravelling the natural history. Time-lapse technic itself is very suited for monitoring changes in time. However, the presented study has limitations and disadvantages. The first limitation is the difference in projection of the wrists. Not all of the radiographs are taken in the exact same position. The position of the wrist and hand vary in several images making it more difficult to compare the successive images. The second limitation is the difference in time in-between the radiographs. Not all intermediate times were similar making the interpretation of the changes difficult.

A clearly disappearing osteochondroma was found in two patients. Yanagawa et al. described the disappearing of osteochondromas in $2001^{30}$. They considered recovery of normal skeletal growth control as most likely mechanism. They further stated that in osteochondromas with fracture an alteration of vascular supply might 
contribute to growth arrest. In the two patients in this study no fracture of the osteochondromas was seen.

A disadvantage of taking multiple radiographs is exposing children to ionizing radiation. Besides the exposure to radiation the radiographs only show a twodimensional image. In future studies the use of standardized MR images of the wrist used in a time-lapse manner could counter these disadvantages. These images could show the epiphyseal cartilage and the deformity of the joints more clearly, making a three-dimensional time-lapse possible. Hopefully this will lead to a better understanding of the developmental deformities in the wrists of $\mathrm{HMO}$ patients.

\section{Conclusion}

Time-lapse technique is suitable to monitor growth of wrists in $\mathrm{HMO}$ patients. It showed no direct relationship between growth of wrists and osteochondromas but it did show osteochondromas disappearing. A steep radial inclination seems related to ulnar shortening and might be due to proximity of radial osteochondromas. Patients had marked radiological changes, but relatively few clinical problems. 


\section{Chapter 8}

\section{REFERENCES}

1. Khurana JA., Osteochondroma. World Health Organization Classification of Tumours. Pathology and Genetics of Tumours of Soft Tissue and Bone., 2002: p.234-36.

2. Bovee JV, Osteochondroma. World Health Organization Classification of Tumours. Pathology and Genetics of Tumours of Soft Tissue and Bone, 2002: p. 360-362.

3. Schmale GA, Conrad EU, Raskind WH, The natural history of hereditary multiple exostoses. J Bone Joint Surg Am, 1994. 76(7): p. 986-92.

4. Legeai-Mallet L, Munnich A, Maroteaux P, Le Merrer, Incomplete penetrance and expressivity skewing in hereditary multiple exostoses. Clin Genet, 1997. 52(1): p. 12-6.

5. Bovee, JV, Multiple osteochondromas. Orphanet J Rare Dis, 2008. 3: p. 3.

6. Goud AL, de Lange J, Scholtes VA, Bulstra SK, Ham SJ. Pain, physical and social functioning, and quality of life in individuals with multiple hereditary exostoses in The Netherlands: a national cohort study.J Bone Joint Surg Am. 2012 Jun 6;94(11):1013-20.

7. Madelung O: Die spontane subluxation de hand nach vorne. Verhandlungen der deutschen Gesellschaft fur Chirurgie 1878;7:259-276

8. Vanhoenacker FM, Van Hul W, Wuyts W, Willems PJ, De Schepper AM. Hereditary multiple exostoses: from genetics to clinical syndrome and complications. Eur J Radiol. 2001 Dec;40(3):208-17.

9. Akita S, Murase T, Yonenobu K, Shimada K, Masada K, Yoshikawa H Long-term results of surgery for forearm deformities in patients with multiple cartilaginous exostoses. Bone Joint Surg Am. 2007 Sep;89(9):1993-9.

10. Gottschalk HP; Kanauchi, Y; Bednar MS; Light, Terry R Effect of osteochondroma location on forearm deformity in patients with multiple hereditary osteochondromatosis. The Journal of hand surgery, 11/2012, Volume 37, Issue 11

11. Burgess RC, Cates $\mathrm{H}$, Deformities of the forearm in patients who have multiple cartilagenous exostosis J Bone Joint Surg, 75A, 1993, pp. 13-18

12. Peterson HA, Deformities and problems of the forearm in children with multiple hereditary osteochondromata J Pediatr Orthop, 14, 1994, pp. 92-100

13. Abe $M$, Shirai $H$, Okamoto $M$, Onomura $T$, Lengthening of the forearm by callus distraction, J Hand Surg, 21B (1996), pp. 151-163

14. Stanton PR, Hansen MO, Function of the upper extremities in hereditary multiple exostoses J Bone Joint Surg, 78A (1996), pp. 568-573 
15. Arms DM, Strecker WB, Manske PR, Schoenecker PL, Management of forearm deformity in multiple hereditary osteochondromatosis J Pediatr Orthop. 1997 Jul-Aug;17(4):450-4.

16. Watts AC, Ballantyne AJ, Fraser M, Simpson HA Porter DE, The Association Between Ulnar Length and Forearm Movement in Patients With Multiple Osteochondromas The Journal of Hand Surgery Volume 32, Issue 5, May-June 2007, Pages 667-673

17. Clement ND, Porter DE, Forearm deformity in patients with hereditary multiple exostoses: factors associated with range of motion and radial head dislocation. J Bone Joint Surg Am. 2013 Sep 4;95(17):1586-92.

18. Solomon L, Bone growth in diaphyseal aclasis, J Bone Joint Surg, 43B, 1961 pp. 700-716

19. Fairbank HAT, Diaphyseal aclasis, J Bone Joint Surg, 31B, 1949, pp.105-113

20. Fogel GR, McElfresh EC, Peterson HA, Wicklund PT, Management of deformities of the forearm in multiple hereditary osteochondromas, J Bone Joint Surg, 66A, 1984, pp. 670680

21. Bader B, Grill F, Ulnar lengthening in osteochondroma of the forearm, Handchir Mikrochir Plast Chir, 32, 2000, pp. 321-327

22. Cheng JC, Distraction lengthening of the forearm J Hand Surg, 16B, 1991, pp. 441-445

23. Dahl MT, The gradual correction of forearm deformities in multiple hereditary exostoses Hand Clin, 9, 1993, pp. 707-718

24. Masada K, Tsuyuguchi Y, Kawai H, Kawabata H, Noguchi K, Ono K, Operation for forearm deformity caused by multiple osteochondromas J Bone Joint Surg, 71A, 1989, pp. 24-29

25. Wood VE, Sauser D, Mudge D, The treatment of hereditary multiple exostosis of the upper extremity, J Hand Surg, 10A, 1985, pp. 505-513

26. Peterson HA, Multiple hereditary osteochondromata, Clin Orthop, 239,1989, pp. 222-230

27. Pritchett JW, Lengthening the ulna in patients with hereditary multiple exostoses, J Bone Joint Surg, 68B, 1986, pp. 561-565

28. Raimondo RA, Skaggs DL, Rosenwasser MP, Dick HM, Lengthening of pediatric forearm deformities using the Ilizarov technique: functional and cosmetic results. J Hand Surg, 24A, 1999, pp. 331-338

29. Rodgers WB, Hall JE, One-bone forearm as a salvage procedure for recalcitrant forearm deformity in hereditary multiple exostoses, J Pediatr Orthop, 13, 1993, pp. 587-591

30. Yanagawa T, Watanabe H, Shinozaki T, Ahmed AR, Shirakura K, et al. (2001) The natural history of disappearing bone tumours and tumour- like conditions. Clin Radiol 56: 877886. 


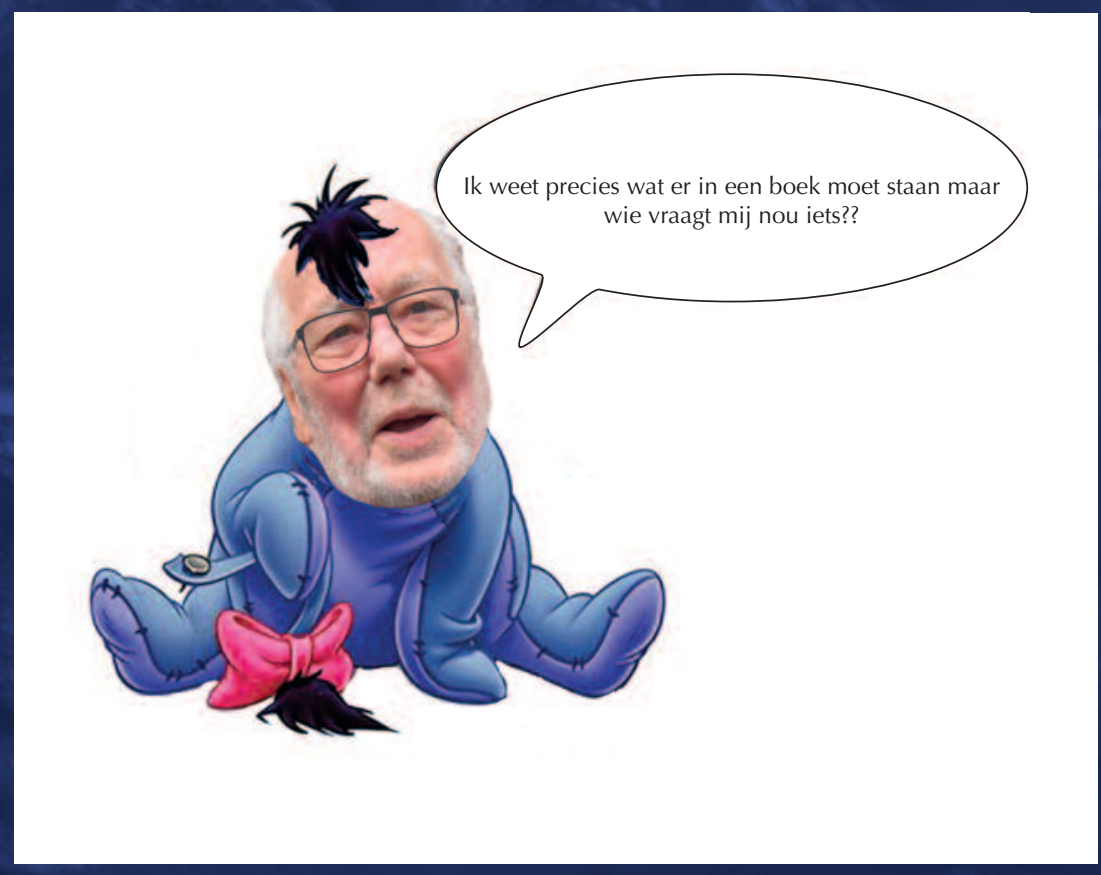




\section{Chapter NINE}

General discussion,

Future direction

and concluding remarks 
Chapter 9 


\section{General Discussion}

In this thesis the natural development and growth of osteochondromas is addressed to increase the understanding of the disease Hereditary Multiple Osteochondroma (HMO). The three main subjects of the thesis are origin and development, structure and clinical visualisation of the osteochondromas.

\section{Origin and development}

Origin. Different hypotheses regarding the formation of osteochondromas have been postulated. The cellular origins that have been suggested for osteochondromas are the perichondrium ${ }^{1,2}$, the groove of Ranvier ${ }^{3-5}$ or the epiphysis ${ }^{6-10}$. In this thesis several chapters support the hypothesis of the epiphysis as the origin of osteochondromas. In chapters one and six is shown that, histologically, the cartilage on top of osteochondromas has a similar structure as epiphyseal cartilage. It consists of separated clusters of chondrocytes in columns, more or less similar to the lobbed histological appearance of the epiphysis. This is supported by Huch et al., their study shows that osteochondromas and normal growth plates share similar proliferation capacity $^{10}$. In chapter three no newly formed osteochondromas proximal to the existing osteochondroma were observed during longitudinal growth of the distal femur, and chapter six shows the presence of hypertrophic chondrocytes staining positive for collagen type $X$, similar to hypertrophic chondrocytes in the epiphysis. Therefore the epiphysis may be a likely place of origin. The osteochondroma as an epiphyseal structure could also explain the growth capacity of osteochondromas in harmony with the growth of the patient ${ }^{11,12}$. No ratification was found for the perichondrium or the groove of Ranvier as place of origin. The perichondrium as a source could not explain how the chondrocytes could gain their proliferative capacity and could not explain the growth of the osteochondromas and the femur both increased over time during growth, as shown in chapter three. In chapter seven no loose cartilage islands were detected in the metaphysis making the perichondrium and the groove of Ranvier an unlikely source. As described by Hameetman et al., the histology of the cells and the fact that the bony stalk grows outside and never in the epiphysis, make these sites unlikely as the pathophysiological source ${ }^{13}$. 


\section{Chapter 9}

Development. Recent advancements in understanding $\mathrm{HMO}$ suggest that aberrant growth factor signalling play a major role in growth of osteochondromas 1,3,5-9,14-18. In $\mathrm{HMO}$ there is a genetic defect of the exostosin (EXT) genes, these genes play an important role in the heparan sulfate (HS) biosynthesis. HSs influence many important processes in skeletal growth and morphogenesis ${ }^{14}$. Studies have indicated that HSs influence the distribution and availability of growth and signalling proteins by influencing the diffusion gradients in the epiphyseal growth plate ${ }^{15}$. Disruption of the diffusion gradient in $\mathrm{HMO}$ (due to aberrant HS presence) likely leads to abnormal proliferation of growth and signalling proteins, including members of the hedgehog (Hh), bone morphogenetic protein (BMP), fibroblast growth factor (FGF) and $\mathrm{Wnt}$ families ${ }^{1,15,18}$. These families of signalling molecules play crucial regulatory roles in epiphyseal skeletal development. This role seems supported by results of the study presented in chapter four. The chapter describes the systemic influence of the disease $\mathrm{HMO}$ on the overall growth rate of the affected individual by determining the skeletal age in relation to the calendar age. The skeletal age in young children with $\mathrm{HMO}$ is lower than their calendar age, while especially for adolescent boys it is higher. This phenomenon may explain diminished stature in $\mathrm{HMO}$ and supports the theory that the systemic influence of the genetic defect leading to osteochondromas may influence the epiphyseal growth. These results are supported by the results of a study performed by Clement et al. in 2012. The results of their study show that the final diminished stature of $\mathrm{HMO}$ patients was relative to their age; in the adolescent age group the stature of $\mathrm{HMO}$ children was taller than that of their peers without the disorder, after the age of 15 they were shorter compared to their peers ${ }^{19}$. Preclinical studies, like the mouse study performed by Koziel et al., that describes a genetic mouse model expressing a truncated form of EXT1, displaying shortened skeletal elements and fused vertebrae, support the theory of the general influence of the gene defect on growth ${ }^{18}$. The general influence on growth is of direct clinical relevance in the planning of epiphysiodesis in leg length discrepancy and hemi-epiphysiodesis in axial deformities, common features in $\mathrm{HMO}$ patients. Until now there is no algorithm available to predict the growth in these patients. Therefore individual longitudinal follow-up of bone growth is advised and care should be taken especially in boys because of possibly earlier than expected closure of their growth plates. 


\section{Structure}

Osteochondromas have a striking structure: the cartilage cap exhibits an intriguing growth plate-like organization and the bony part of osteochondromas grows at an average angle of 60 degrees to the normal growth direction of the bone $\mathrm{e}^{20,21}$. Amongst other, because of this off-axis position osteochondromas are expected to carry fewer loads than normal bone. Chapter five of this thesis addresses the micro architecture of the bone compartment of the osteochondromas. The chapter investigates the hypothesis that osteochondromas present with a less developed microstructure than normal developing trabecular bone. This was investigated by determining the bone morphology of osteochondromas using micro-CT scanning. The results of this pilot study show that osteochondromas have thicker and wider spaced trabeculae with a relatively normal trabecular number and a normal bone volume fraction in the osteochondromas compared to normal trabecular bone of children in the same age range. Since this is the first time a study of the microarchitecture of human osteochondromas was conducted using micro-CT, no comparable values were available. However, a mouse study performed by Sgariglia et al. describes a significantly larger number of osteoclasts and deranged trabecular bone formation in EXT1 deficient mice ${ }^{22}$. If human osteochondromas have a larger number of osteoclasts this might be the cause for the special architecture found in this study. Possibly, these abnormally formed trabeculae can carry smaller load and therefore osteochondromas can spontaneously fracture ${ }^{23-26}$.

Apart from the lesser load, the cause of this abnormal trabecular bone formation may originate from the abnormal heparan sulphate-reduced biological environment of the osteochondromas. The cartilage tissue on top of the osteochondromas forms the trabeculae, probably by endochondral ossification. Chapter six compares the histology of this epiphyseal-like chondrocyte cap, to the histology of the articular cartilage cells in Dysplasia Epiphysealis Hemimelica (DEH). In literature, DEH is described as an epiphyseal osteochondroma or as an osteochondroma-like lesion based on histological evaluation. Both diseases are seen as different entities only based on their different location and appearance, thereby implying a common aetiology ${ }^{27-35}$. Chapter six shows that even if DEH and HMO are histologically comparable on a cellular level, their biological activity seems 


\section{Chapter 9}

different. For example the immunostaining for collagen type $X$, stained positive in the hypertrophic chondrocytes of the osteochondromas and it was not able to detect any staining in DEH chondrocytes.

\section{Visualisation}

Osteochondromas are easily detected on conventional radiographs. Nevertheless, the radiographs only show the calcified bony stalk of osteochondromas. The top of osteochondromas, and at younger age the complete osteochondroma, is cartilaginous and therefore not visible using conventional radiography. Because osteochondromas grow and influence their surrounding tissues, they can lead to compression of tendons, nerves, muscles, ligaments and of the spinal $\operatorname{cord}^{36,37}$. Therefore there is a need to increase the visibility of osteochondromas in two areas: the visibility of the soft tissues and the monitoring of growth. This way complication might be prevented, for example, by prior resection of the osteochondroma. Chapter seven describes a study in which Whole body MR Imaging ( $\mathrm{Wb} \mathrm{MRI}$ ) was used as an imaging technique in HMOaffected paediatric patients. Wb MRI showed accurate detectability of osteochondromas. It potentially reduces the exposure to ionising radiation and may lead to early detection of osteochondromas compared to conventional radiography. Until now there are no Wb MRI protocols available for HMO. In our approach the proton density SPIR setting showed the best visibility of osteochondromas. Therefore this setting is advised for Wb MRI in children with HMO. To subsequently follow growth, the Wb MRI without a standardised patient positioning protocol is not suitable. In order to follow growth in time chapter eight describes the use of time-lapse technique on wrists of paediatric patient with $\mathrm{HMO}$. About half of the $\mathrm{HMO}$-affected patients develop a Madelung-like deformity at their wrists ${ }^{11,12,21,36-38}$. This deformity develops slowly over time and the best way to visualise the slow development is the time-lapse technique. The results of the study indicate that in some patients a gradual shorting of the ulna does not directly depend on the growth of osteochondromas. The radial deformity seems more dependent on the proximity of radial osteochondromas. The time-lapse technique is suited for monitoring changes in time. However, since the radiographs only show the bony part, a three-dimensional time-lapse method following standardized MR images would provide a better image and possibly lead to better understanding of the developmental deformities in $\mathrm{HMO}$ patients. 
Of course the drive of a paediatric orthopaedic surgeon consulting children with $\mathrm{HMO}$ is to find a treatment. Hopefully early detection of osteochondromas and associated deformities can lead to an early intervention in order to prevent deformities that later need to be corrected surgically.

\section{FUTURE DIRECTION AND CONCLUDING REMARKS}

This thesis describes different aspects of the natural development and growth of osteochondromas, with three main subjects: origin and development, structure, and visualisation of the osteochondromas. The structure, origin and development are possibly due to the genetics of the disease. The exostosin (EXT) genes play a role in the heparin sulphate (HS) biosynthesis. The absence of HS seems to disturb the major regulatory systems of epiphyseal growth and subsequently assists in the development of the osteochondromas. Studies show that $\mathrm{HMO}$ patients suffer from a variety of less obvious problems that can include wound healing delay, general laxity, learning disabilities and dental problems ${ }^{39,40}$. These findings can relate to the HS's general influence. Besides these non-skeletal features in patients suffering from $\mathrm{HMO}$, patients suffering from other genetic diseases can have multiple osteochondromas as investigated by Morales-Piga et $a{ }^{4}{ }^{41}$. They studied a group of patients with fibrodysplasia ossificans progressiva (FOP) phenotype and found metaphyseal osteochondromas as a very frequent trait of FOP. Mutations in the ACVR1 gene are associated with FOP. The ACVR1 gene encodes a type I receptor for bone morphogenetic proteins (BMPs), underlining the crosslinking between the different regulatory systems. Unveiling the links between these pathways could help to illuminate the mechanisms that govern bone morphogenesis.

Future studies could be conducted to elaborate on these pathways and could direct towards the understanding of the metabolism of cartilage in HMO. For instance, the biological activity of the cartilage of the epiphysis can be compared to the cartilage on top of an osteochondroma and articular cartilage using the Delayed ContrastEnhanced MRI (dGEMRIC), to study cartilage glycosaminoglycan (GAG) content in vivo. 


\section{Chapter 9}

Another way to study cell function has been described by Frost et al. ${ }^{42}$ : Administration of tetracyclines can be used to visualize bone metabolism. Administering tetracycline as a bone fluorophor, in different doses and at different timeframes, allows for a comparison of the ingrowth of the tetracycline in the developmental areas of bony tissue. Therefore it is expected to provide insight into the development of the bony part of the osteochondroma from the cartilage top in comparison to the formation of skeletal-bone from the epiphyseal cartilage.

In addition, great progress has been made over the years in genome sequencing. A recent study by Sgariglia et al. in $213 \mathrm{HMO}$ patients and 1890 controls showed a significant underrepresentation of $\mathrm{T}$ allele in the $\mathrm{HMO}^{43}$. It is suggested that modifier genes play a role in determining the severity of the disease because of wide clinical heterogeneity. The data by Sgariglia indicate that there is a possible genetic connection between TCF7L2 and EXT. These loci could possibly modulate shared pathways and can be further studied in the future.

The first study into the bone structure of human osteochondromas is described in this thesis ${ }^{44}$. In this study the microarchitecture of the osteochondromas is demonstrated; showing less, but thicker and wider spaced trabeculae with a relatively normal bone volume. Future studies are directed toward the use of the HR-pQCT (XtremeCT) for imaging the osteochondromas in vivo and to visualize the adjacent long bone. This is expected to give insight into the structure and quantifies the differences between the host bone and the osteochondroma. If the study is conducted longitudinally in growing children, a quantified measure of growth could potentially be identified.

The recent possibility to study MR images in a three-dimensional manner creates the opportunity to study the cartilage and bone of osteochondromas in a time-lapse manner. It also opens doors to volume measurements. Volume measurement, of for example cartilage cap in consecutive MR images, could demonstrate the growth of the cartilage compartment of the osteochondroma.

An important issue to be addressed in the future is whether and how osteochondroma formation can be prevented or even reversed therapeutically. At present symptomatic osteochondromas are removed surgically. Surgery can be dangerous and can have serious complications. Therefore biological or minimal invasive surgical solutions are needed. A better understanding of the origin, the 
biology and the influence on the surrounding tissues might aid in finding an early treatment for osteochondromas and $\mathrm{HMO}$. Thoughts about minimal or non-invasive treatment by cryosurgery or laser treatment come to mind. Further along maybe genetic or biological manipulation by specific biological or gene therapy would be possible.

This thesis describes the natural development of osteochondromas in HMO. Since $\mathrm{HMO}$ is a genetic disorder the systemic influence of the disease is responsible for many of its features. In the future the missing link between the affected gene loci and the underlying pathways could be uncovered by a better understanding of heparan sulphate influence on the signaling proteins. The use of genome sequencing, the administration of tetracyclines and Delayed Contrast-Enhanced MRI technique may further help to unravel this knowledge hiatus.

The thesis further shows the general influence on the maturation of the growth plate leading to the shorter stature and possibly leading to the growth deformities associated with $\mathrm{HMO}$. Individual radiological monitoring of patients' bone age and the development of growth deformities is therefore advised. In the future Whole body MRI screening could replace radiological monitoring. Three dimensional imaging techniques may aid in the search for early interventions, making minimal or non invasive treatment by cryosurgery or laser treatment possible.

Gain in these fields is expected to increase understanding of osteochondroma growth patterns and may help in the search for early diagnosis and early treatment.

Heleen M. Staal 


\section{Chapter 9}

\section{REFERENCES}

1. Jones, KB, Morcuende JA, Of hedgehogs and hereditary bone tumors: reexamination of the pathogenesis of osteochondromas. lowa Orthop J, 2003. 23: 87-95.

2. Muller, Uber hereditare multiple cartilaginare exostosen und ecchondrosen. Beitr Pathol Anat 1914, 57: 232.

3. Hecht JT, Hayes E, Haynes R, Cole WG, Long RJ, Farach-Carson MC, Carson DD, Differentiation-induced loss of heparan sulfate in human exostosis derived chondrocytes. Differentiation. 2005 Jun;73(5):212-21.

4. Porter DE, Simpson AH, The neoplastic pathogenesis of solitary and multiple osteochondromas.J Pathol. 1999 Jun;188 (2):119-25.

5. Mansoor A, Beals RK, Multiple exostosis: a short study of abnormalities near the growth plate. J Pediatr Orthop B, 2007. 16(5): p. 363-5.

6. Jones KB, Piombo V, Searby C, Kurriger G, Yang B, Grabellus F, Roughley PJ, Morcuende JA, Buckwalter JA, Capecchi MR, Vortkamp A, Sheffield VC, A mouse model of osteochondromagenesis from clonal inactivation of Ext1 in chondrocytes, Proc Natl Acad Sci U S A. 2010 Feb 2;107(5):2054-9.

7. Matsumoto K, Irie F, Mackem S, Yamaguchi Y, Ruoslahti E, A mouse model of chondrocytespecific somatic mutation reveals a role for Ext1 loss of heterozygosity in multiple hereditary exostoses. Proc Natl Acad Sci U S A. 2010 Jun 15;107(24):10932-7

8. Zak BM, Schuksz M, Koyama E, Mundy C, Wells DE, Yamaguchi Y, Pacifici M, Esko JD. Compound heterozygous loss of Ext1 and Ext2 is sufficient for formation of multiple exostoses in mouse ribs and long bones. Bone. 2011 May 1;48 (5):979-87.

9. Sgariglia F, Candela ME, Huegel J, Jacenko O, Koyama E, Yamaguchi Y, Pacifici M, Enomoto-Iwamoto M. Epiphyseal abnormalities, trabecular bone loss and articular chondrocyte hypertrophy develop in the long bones of postnatal Ext1-deficient mice. Bone. 2013 Nov;57(1):220-31.

10. Huch K, Mordstein V, Stöve J, Nerlich AG, Amholdt H. Delling, G; Puhl, W; Günther, KP; Brenner, RE. Expression of collagen type I, II, X and Ki-67 in osteochondroma compared to human growth plate cartilage. Eur J Histochem 2002 46: 249-258.

11. Schmale GA,.Conrad EU, Raskind WH, The natural history of hereditary multiple exostoses. J Bone Joint Surg Am, 1994. 76(7): p. 986-92. 
12. Kitsoulis P, Vassiliki G, Kalliopi S, Georgios P, Georgios K, Niki JA, Maria B. Osteochondromas: review of the clinical, radiological and pathological features. In Vivo, 2008. 22(5): p. 633-46.

13. Hameetman L, Szuhai K, Yavas A, Knijnenburg J, van Duin M. The role of EXT1 in nonhereditary osteochondroma: identification of homozygous deletions. J Natl Cancer Inst 2007, 99: 396-406.

14. Huegel J, Sgariglia F, Enomoto-Iwamoto M, Koyama E, Dormans JP,Pacifici M, Heparan Sulfate in Skeletal Development, Growth, and Pathology: The Case of Hereditary Multiple Exostoses Dev Dyn. 2013 Sep;242(9):1021-32.

15. de Andrea CE, Prins FA, Wiweger MI, Hogendoorn PC, Pancras CW. Growth plate regulation and osteochondroma formation: insights from tracing proteoglycans in zebrafish models and human cartilage. J Pathol 2011, 224: 160-168.

16. Kronenberg HM. Developmental regulation of the growth plate. Nature. 2003, May 15; 423(6937):332-6.

17. Häcker U, Nybakken K, Perrimon N. Heparan sulphate proteoglycans: the sweet side of development Nat Rev Mol Cell Biol. 2005 Jul;6(7):530-41.

18. Koziel L, Kunath M, Kelly OG, Vortkamp A. Ext1-dependent heparan sulfate regulates the range of Ihh signaling during endochondral ossification. Dev Cell. 2004 Jun;6(6):801-13.

19. Clement ND, Duckworth AD, Baker AD, Porter DE. Skeletal growth patterns in hereditary multiple exostoses: a natural history. J Pediatr Orthop B. 2012 Mar;21(2):150-4.

20. Milgram JW, The origins of osteochondromas and enchondromas. A histopathologic study. Clin Orthop Relat Res 1983: 264-284.

21. Vanhoenacker FM, Van Hul W, Wuyts W, Willems PJ, De Schepper AM Hereditary multiple exostoses: from genetics to clinical syndrome and complications. Eur J Radiol 2001, 40: 208-217.

22. Sgariglia F, Candela ME, Huegel J, Jacenko O, Koyama E, Yamaguchi Y, Pacifici, M, Enomoto-Iwamoto M, Epiphyseal abnormalities, trabecular bone loss and articular chondrocyte hypertrophy develop in the long bones of postnatal Ext1-deficient mice. Bone 2013, 57: 220-231.

23. Henry NE, Carow SD, Kocher BL. Osteochondroma fracture at the distal medial femur. J Orthop Sports Phys Ther 2014, 44: 457. 


\section{Chapter 9}

24. Kanauchi T, Suganuma J, Kawasaki T, Mochizuki R, Inoue Y. Fracture of an osteochondroma of the femoral neck caused by impingement against the ischium. Orthopedics 2012, 35: e1438-1441.

25. Kose O, Ertas A, Celiktas M, Kisin B. Fracture of an osteochondroma treated successfully with total excision: two case reports. Cases 2009, J 2: 8062.

26. Theodorou SD, Karamitsos S, Tsouparopoulos D, Hatzipavlou AG. Rare complications of exostosis. Fractures and injury to the common peroneal nerve. Acta Orthop Belg 1978, 44: 496-505.

27. Ouyang Z, Xu M, Li X, Peng D. Dysplasia epiphysealis hemimelica with involvement of the distal tibial epiphysis and talus: recurrence of a case and literature review. The Journal of foot and ankle surgery : official publication of the American College of Foot and Ankle Surgeons. 2014 Mar-Apr;53(2):199-202.

28. Wiart E, Budzik JF, Fron D, Herbaux B, Boutry N. Bilateral dysplasia epiphysealis hemimelica of the talus associated with a lower leg intramuscular cartilaginous mass. Pediatric radiology. 2012 Apr;42(4):503-7.

29. Takeuchi H, Ito K, Ogino T, Hasegawa T, Kitamura M, Ishii S. A case of osteocartilaginous mass involving the coronoid process of the ulna: solitary osteochondroma or dysplasia epiphysealis hemimelica? Journal of shoulder and elbow surgery / American Shoulder and Elbow Surgeons. 2003 Sep-Oct;12(5):510-3.

30. Vogel T, Skuban T, Kirchhoff C, Baur-Melnyk A, Siegert S, Heimkes B. Dysplasia epiphysealis hemimelica of the distal ulna: a case report and review of the literature. European journal of medical research. 2009 Jun 18;14(6):272-6.

31. Vanhoenacker F, Morlion J, De Schepper AM, Callewaert E. Dysplasia epiphysealis hemimelica of the scaphoid bone. European radiology. 1999;9(5):915-7.

32. Shinozaki T, Ohfuchi T, Watanabe H, Aoki J, Fukuda T, Takagishi K. Dysplasia epiphysealis hemimelica of the proximal tibia showing epiphyseal osteochondroma in an adult. Clinical imaging. 1999 May-Jun;23(3):168-71.

33. Takagi M, Kiyoshige $Y$, Ishikawa A, Ogino T. Multiple occurrence of osteochondromas in dysplasia epiphysealis hemimelica. Archives of orthopaedic and trauma surgery. 2000;120(5-6):358-60.

34. Azzoni R. Dysplasia epiphysealis hemimelica of the talus. Journal of orthopaedics and traumatology : official journal of the Italian Society of Orthopaedics and Traumatology. 2009 Mar;10(1):43-6. 
35. Bosch C, Assi C, Louahem D, Alkar F, Mazeau P, Delfour C, Canavese, F, Prodhomme, O, Cottalorda, J. Diagnosis and surgical treatment of dysplasia epiphysealis hemimelica. A report of nine cases. Orthopaedics \& traumatology, surgery \& research : OTSR. 2014 Dec;100(8):941-6.

36. Bovee, JV, Multiple osteochondromas. Orphanet J Rare Dis, 2008. 3: p. 3.

37. Goud AL, de Lange J, Scholtes VA, Bulstra SK, Ham SJ. Pain, physical and social functioning, and quality of life in individuals with multiple hereditary exostoses in The Netherlands: a national cohort study. J Bone Joint Surg Am. 2012 Jun 6;94(11):1013-20.

38. Madelung O: Die spontane subluxation de hand nach vorne. Verhandlungen der deutschen Gesellschaft fur Chirurgie 1878;7:259-276

39. Hosalkar H, Greenberg J, Gaugler RL, Garg S, Dormans JP. Abnormal scarring with keloid formation after osteochondroma excision in children with multiple hereditary exostoses. J Pediatr Orthop. 2007 Apr-May;27(3):333-7.

40. Wiweger MI, Zhao Z, van Merkesteyn RJ, Roehl HH, Hogendoorn PC. HSPG-deficient zebrafish uncovers dental aspect of multiple osteochondromas. PLoS One. 2012;7(1):e29734.

41. Morales-Piga A, Bachiller-Corral J, González-Herranz P, Medrano-Sanlldelfonso M, Olmedo-Garzón J, Sánchez-Duffhues G. Osteochondromas in fibrodysplasia ossificans progressiva: a widespread trait with a streaking but overlooked appearance when arising at femoral bone end. Rheumatol Int. 2015 Oct;35(10):1759-67.

42. Frost HM.Tetracycline-based histological analysis of bone remodeling. Calcif Tissue Res. 1969;3(3):211-37

43 Sgariglia F, Pedrini E, Bradfield JP, Bhatti TR, D'Adamo P, Dormans JP, Gunawardena AT, Hakonarson H, Hecht JT, Sangiorgi L, Pacifici M, Enomoto-Iwamoto M, Grant SF. The type 2 diabetes associated rs7903146 T allele within TCF7L2 is significantly under-represented in Hereditary Multiple Exostoses: insights into pathogenesis. Bone. 2015 Mar;72:123-7.

44. Staal HM, Nijhuis W, Witlox MA, Boom KRJ, Paffen G, Ham SJ, Welting TJM, van Rietbergen B, van Rhijn LW The microarchitecture of bone in osteochondromas, a pilot study with micro computed tomography imaging. Journal of Bone Reports and Recommendations, 2015. Vol. 1 No. 2:10 


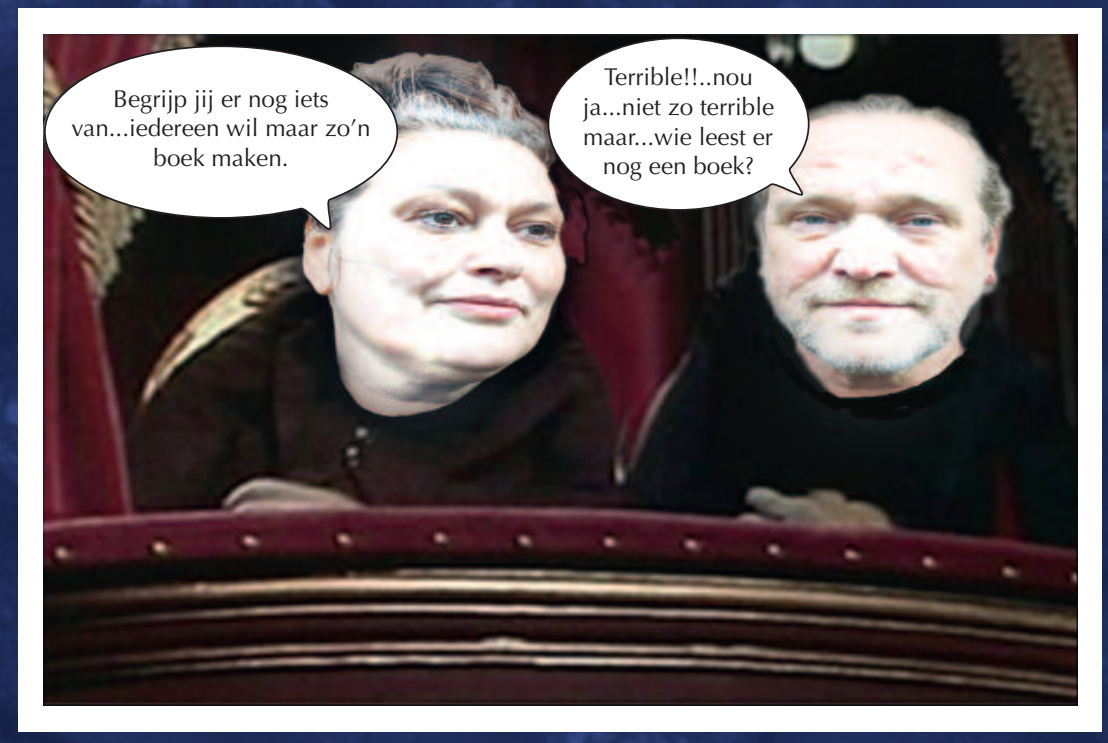




\section{Chapter ten}

Summary

Valorisation

Nederlandse samenvatting 
Chapter 10 


\section{SUMMARY}

In this thesis the natural development and growth of osteochondromas is addressed to increase the understanding of the disease Hereditary Multiple Osteochondroma ( $\mathrm{HMO})$. The subjects described are origin and development, structure and clinical visualisation of osteochondromas.

Origin and Development: In the past different hypotheses regarding the formation of osteochondromas have been postulated. Studies presented in this thesis support the hypothesis of the epiphysis as the origin of osteochondromas, because of two reasons; firstly because of the histological comparison between osteochondroma cartilage and epiphyseal cartilage and secondly because growth patterns of osteochondromas equal growth patterns of the epiphysis in individual patients. Development of osteochondromas seems to be directed by growth hormones. Since $\mathrm{HMO}$ is a genetic disorder, the systemic influence of the genetic abnormality may be responsible for many $\mathrm{HMO}$-related clinical features, for example the general influence of $\mathrm{HMO}$-associated gene mutations on the maturation of the growth plates. Genetic mutations in the exostosin genes lead to abnormal chondrocyte differentiation and proliferation, due to aberrant expression and function of growth factors and other signaling molecules. Families of these signaling molecules play crucial regulatory roles in epiphyseal skeletal development. This may result in decreased stature and growth deformities that are typically associated with HMO. Data presented in this thesis show that the skeletal age in young children with $\mathrm{HMO}$ is lower than their calendar age, while for adolescent boys it is higher. This general influence on growth is of direct clinical relevance in the planning of epiphysiodesis in leg length discrepancy and hemi-epiphysiodesis in axial deformities, which are common features in $\mathrm{HMO}$ patients. Until now there is no algorithm available to predict the growth in these patients, therefore individual monitoring of patients' bone age and the development of growth deformities is advised.

Structure: The microstructure of osteochondromas is addressed in a pilot study. It shows that osteochondromas have thicker and wider spaced trabeculae. This is combined with a relatively normal trabecular number and a normal bone volume fraction, compared to normal trabecular bone of children in the same age range. 


\section{Chapter 10}

These observations may provide an explanation why osteochondromas have an osteoporotic-like appearance and why they can spontaneously fracture. In future a better understanding of the microstructure might potentially lead to early interventions to influence the growth of the osteochondromas.

Clinical visualisation: Conventional radiographs clearly show the bony part of osteochondromas but fail to visualize the cartilaginous cap. Because osteochondromas grow, they can compress surrounding tissues. As the cartilaginous cap is a substantial part of the osteochondroma, especially in children, there is a need to increase the visibility of the cartilaginous part of osteochondromas as well. A study using Whole body MR (Wb MRI) with proton density SPIR setting showed accurate detectability of osteochondromas based on the clear visualisation of the cartilaginous cap. The use of MR imaging reduces the exposure to ionising radiation and it may lead to early detection of osteochondromas compared to conventional radiography. Another way to improve the visibility of the growth of osteochondromas over time could be the use of time-lapse technique. This technique enhances the visibility of slow changes over time. It is suitable to monitor growth of wrists in HMO patients, but showed no direct relationship between growth of wrists and osteochondromas. It did show however osteochondromas disappearing, demonstrating that not all osteochondromas remain.

Future studies are directed towards the use of the HR-pQCT (XtremeCT) for imaging the osteochondromas in vivo. Furthermore the missing link between the affected gene loci and the underlying pathways could be uncovered through a better understanding of the role of heparan sulphate chains in sequestering of signaling proteins.

Progress in these fields is expected to increase understanding of osteochondroma growth patterns and may help in the search for early diagnosis and early treatment. 


\section{VALORISATION}

In this thesis the natural development and growth of osteochondromas is addressed to increase the understanding of the disease Hereditary Multiple Osteochondroma $(\mathrm{HMO})$. The three subjects described are origin and development, structure and clinical visualisation. Osteochondromas are defined as cartilage-capped bony outgrowths on the surface of long bones containing a marrow cavity that is continuous with that of its underlying host bone ${ }^{1,2}$. They are usually localized near the metaphysis of bones that develop by endochondral ossification ${ }^{3,4}$. In theory every bone element that is formed by endochondral bone formation is thought to be susceptible for osteochondroma formation.

In this thesis the first study into the bone structure of human osteochondromas is described (chapter five). By using Micro-Ct imaging in vitro, the structure of the bone morphology was visualized. It showed a difference between bone morphology of children and the morphology of the osteochondromas. The bone structure of osteochondromas showed thicker and wider spaced trabeculae. This wider spacing could possibly explain the osteoporotic-like in vivo structure and might also explain the tendency of osteochondromas to easily fracture. These observations might lead the way in future studies, directed toward the use of the HR-pQCT (XtremeCT) for imaging the bone structure of osteochondromas in vivo. With the HR-pQCT we might be able to visualize the difference in bone formation of the host bone and the adjacent osteochondroma. Which might give a hint on why some osteochondromas subsist and others disappear, possibly giving a clue on how to intervene in an early stage.

For a better understanding of the natural growth of $\mathrm{HMO}$ patients, two radiological studies were conducted. Both studies (chapters three and four) showed that growth of osteochondromas is linked to general skeletal growth of individuals. Chapter four further shows a discrepancy, especially in adolescent boys, between the skeletal and the calendar age. This observation is of relevance to patients with $\mathrm{HMO}$ because leg length discrepancy and axial deviation are common in $\mathrm{HMO}^{5,6}$. These deformities can be treated operatively to guide the patient's growth ${ }^{7}$. In this 


\section{Chapter 10}

kind of surgical intervention it is of utmost importance to know the remainder of growth, to calculate the best possible moment to intervene in order to correct the deformity at best. The presented findings show that the prediction models for normal growth in children do not apply to children with HMO. This implies that for timing of surgery in children with $\mathrm{HMO}$, it is essential to use individual growth curves of each individual patient to predict their growth and not the broadly used general growth predictors of normal children. Regular bone age tests, height and bone-length measurements are therefore advised in children with $\mathrm{HMO}$.

To follow the skeletal growth and bone deformity formation in $\mathrm{HMO}$ patients in current practice, subsequent radiographs are used. The results presented in chapter seven show an alternative with the use of Whole body MRI. Using the Whole body MRI to subsequently follow growth in HMO patients has clear advantages: less radiation is needed, soft tissues can be visualized and costs can be reduced. Subsequent radiographs of all involved bones in severe $\mathrm{HMO}$ outweigh the costs of Whole body MRI. The proton density SPIR setting showed the best visibility of osteochondromas in Whole body MRI, the cartilage cap clearly visible and was even more intensely visible than articular cartilage. Therefore this setting is advised for Whole body MR imaging in children with HMO. In future, with the right setting and scanning protocol and shorter scanning time, Whole body MRI screening can replace conventional radiological monitoring in the future.

Deformities in HMO develop slowly over time. Therefore the presently used conventional radiographs can be used in a clinically more informative manner by projecting the radiographs in a time lapse (chapter eight). In this way the changes over time in the development of osteochondromas and their surrounding anatomical structures become more evident and may be clinically more easily interpretable. Knowledge about these growth changes may aid in the search for early interventions in osteochondroma formation and in the possible interventions in order to prevent deformities of the host bone or surrounding tissues.

Increased understanding of osteochondroma growth patterns and host bone development may help in the search of early diagnosis and early treatment of 
osteochondromas. This might make minimal or non-invasive treatment possible in the future. Treatment opportunities like for instance cryosurgery or laser treatment in an early stage of the development of the cartage cap could be thought of. Furthermore the work in this thesis may contribute to the development of universal follow-up protocols on how to follow growth in $\mathrm{HMO}$ patients, aiding clinicians and patients in foreseeing deformity formation and planning the timing of surgical treatment.

\section{ReferenCeS}

1. Khurana J, A.-K.F.a.B.J, Osteochondroma (2002) World Health Organization Classification of Tumours. Pathology and Genetics of Tumours of Soft Tissue and Bone: 234-236.

2. Bovee JVMG (2002) HP Osteochondroma. World Health Organization Classification of Tumours. Pathology and Genetics of Tumours of Soft Tissue and Bone.

3. Schmale GA, Conrad EU 3rd, Raskind WH (1994) The natural history of hereditary multiple exostoses.J Bone Joint Surg Am 76: 986-992.

4. Kitsoulis P, Galani V, Stefanaki K, Paraskevas G, Karatzias G, et al. (2008) Osteochondromas: review of the clinical, radiological and pathological features.In Vivo 22: 633-646.

5. Nawata K, Teshima R, Minamizaki T, Yamamoto K (1995) Knee deformities in multiple hereditary exostoses. A longitudinal radiographic study.ClinOrthopRelat Res : 194-199.

6. Porter DE, Emerton ME, Villanueva-Lopez F, Simpson AH. Clinical and radiographic analysis of osteochondromas and growth disturbance in hereditary multiple exostoses. J Pediatr Orthop 2000;20: 246-50.

7. Tompkins M, Eberson C, Ehrlich M. Hemiepiphyseal stapling for ankle valgus in multiple hereditary exostoses. Am J Orthop (Belle Mead NJ) 2012;41: E23-6. 


\section{Chapter 10}

\section{SAmenvatting}

Dit proefschrift is geschreven om het ziekteproces van de ziekte Hereditaire Multiple Osteochondromen ( $\mathrm{HMO}$ ) beter te begrijpen. Osteochondromen zijn benige botuitsteeksels, bekleedt met een kraakbeenkap. Er is gekeken naar opvallende aspecten van de natuurlijke groei en ontwikkeling van osteochondromen. Het meest in het oog springend is het "tegen de richting in" groeien, anders dan alle andere botten in het lichaam die de assen van de belasting volgen. De osteochondromen maken een hoek van gemiddeld 60 graden op deze belastingsas. Onbelast bot wordt in het algemeen geresorbeerd en zal dus langzaam verdwijnen. Wat bij osteochondromen opvalt is dat ze dus buiten de belastingsas groeien, maar niet allemaal verdwijnen. Ondanks de kennis die we hebben over de genetische afwijking bij $\mathrm{HMO}$, is het nog steeds onduidelijk waaruit de osteochondromen ontstaan. Om deze aspecten te belichten geeft het tweede hoofdstuk een overzicht van de literatuur. Bij HMO zijn de exostosin (EXT) genen gemuteerd. De producten van deze genen hebben een rol in de synthese van heparan sulfaat (HS). De afwezigheid van HS lijkt de belangrijkste regulerende systemen van de groeischijf te verstoren, waardoor de ontwikkeling van osteochondromen mogelijk is.

De top van een osteochondroom bestaat uit een kraakbenige kap. Deze kap wordt gevormd door kraakbeencellen die overeenkomsten vertonen met de kraakbenige cellen van een groeischijf, daarom is gekeken naar de groei van osteochondromen in relatie tot de normale bot-groei. De groei van osteochondromen van het bovenbeen houdt gelijke tred met de normale groei van het bovenbeen (hoofdstuk 3). Het toont bovendien dat er geen nieuwe osteochondromen gevormd worden proximaal van de bestaande osteochondromen. Het lijkt aannemelijk dat osteochondromen worden gevormd op of nabij de groeischijf.

We beschrijven de systemische invloed van de ziekte $\mathrm{HMO}$ op de groeisnelheid door bepaling van de skeletleeftijd ten opzichte van de kalenderleeftijd (hoofdstuk 4). De skeletleeftijd bij jongere kinderen met HMO is lager dan hun kalenderleeftijd, terwijl het voor adolescenten omgekeerd is; zij zijn dus sneller 
uitgegroeid. Dit verschijnsel kan de geringere lengte van volwassenen met $\mathrm{HMO}$ verklaren. Het ondersteunt de theorie dat de systemische invloed van het genetisch defect dat tot osteochondromen leidt, ook de groeischijf beïnvloedt.

In het proefschrift wordt verder de architectuur bestudeerd van de osteochondromen, zowel het benige als het kraakbenige deel. De benige opbouw wijkt af van normaal bot, de botbruggen zijn breder en staan wijder van elkaar, terwijl het totale botvolume ongeveer gelijk is aan normaal bot (hoofdstuk 5). De kraakbeencellen lijken op de normale kraakbeencellen in een groeischijf, maar de organisatie van de cellen is anders; in de groeischijf liggen de cellen in rijen, terwijl in de osteochondromen de cellen geclusterd zijn in lobben (hoofdstuk 6).

Daarnaast worden twee technieken beschreven om de osteochondromen bij kinderen in beeld te brengen. $\mathrm{Er}$ is gebruik gemaakt van een totale-lichaams-MRI (hoofdstuk 7). Met behulp van deze scantechniek, waarbij het gehele lichaam wordt afgebeeld, kunnen alle osteochondromen worden gedetecteerd (kraakbeen en bot). Het voordeel van het gebruik van de MRI-scan is dat de stralenbelastende röntgenfoto's bij kinderen niet meer nodig zijn. Het nadeel is dat kleinere kinderen eventueel onder narcose moeten worden gebracht om de scan te vervaardigen. Het groeiproces van osteochondromen bij de pols wordt gevisualiseerd met de timelapse techniek, waardoor het langzame groeiproces en de groeistoornissen versneld zichtbaar worden. Zo ontstaat er een beter beeld van de ontwikkeling van osteochondromen en van groeistoornissen van het onderliggende bot (hoofdstuk 8).

Het proefschrift sluit af met een aantal ideeën voor toekomstige studies. Met de HR-pQCT (XtremeCT) kan onderzoek worden gedaan naar de structuur en groei van het benige deel in vivo. Onderzoek naar het metabolisme van kraakbeen in de kap met MRI techniek of histologisch onderzoek kan mogelijk meer inzicht geven in het cellulaire aspect van HMO. Het werk beschreven in dit proefschrift geeft een beter begrip van de groei van osteochondromen en van het onderliggende bot. In de toekomst kan het helpen in de zoektocht naar een vroegtijdige behandeling van $\mathrm{HMO}$. 


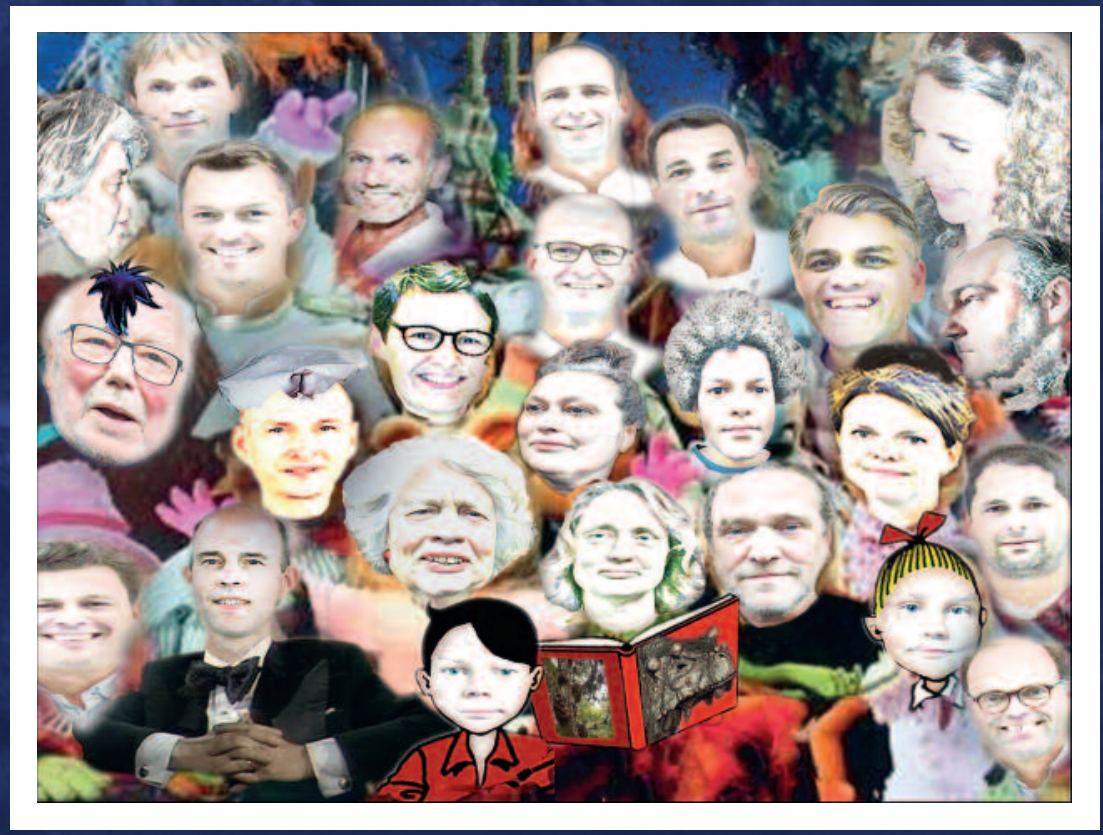




\section{APPENDIX}

Curriculum vitae

List of publications

Dankwoord 
Appendix 


\section{Curriculum vitae}

Heleen Muriel Staal was born on Sunday April $19^{\text {th }} 1970$ in Maastricht. She attended St-Maartens College in Maastricht. In 1988 she started to study medicine at the Catholic University of Nijmegen. In 1996 she received a master degree in Medicine, Faculty of Medicine, with a minor in Tropical Medicine (Faculty of Medicine, Managua, Nicaragua) and Applied Mathematics (Faculty of Mathematics and Computer Science, Nijmegen).

Between 2001 and 2007 she did her post-academic education to become an orthopedic surgeon in respectively Sophia Hospital Zwolle, head Dr. JE. de Vries; UMCU Utrecht, head Prof. RM. Castelein and Prof. AJ. Verbout; VUMC Amsterdam, head Prof. PJJM. Wuisman and Prof. BJ. van Royen and OLVG Amsterdam, head Dr. WJ. Willems.

Since 2007 she has been working as an orthopedic surgeon at MUMC Maastricht, specialized in child -orthopedics and hand surgery. She has a special interest in medical education and holds an academic educational qualification (BKO). In 2012 she became tutor of trainee orthopedic surgeons.

She has conducted her research at CAPHRI School for Public Health and Primary Care. 
Appendix 


\section{Publications}

Slappendel R, Staal HM, Oosterhof J, Egmond J v, Crul BJP. Transcutane elektrische zenuwstimulatie bij patiënten met ernstige chronische cervicobrachialgie. NTPP, 1996; 16 (4).

Staal HM, Hubach PCG. Behandeling van ernstige reumatische artritis van de pols met de Guepar totale pols prothese. Nederlands Tijdschrift voor Geneeskunde, 1998; 142 (50): 2766. (abstract).

Lieshout ON, Staal HM, Wuisman PIJM, Orthokwis, Nederlands Tijdschrift voor Orthopaedie, 1999; 6.

Wuisman PIJM, Dijk M v, Staal HM, Royen BJ v. Augmentation of (pedicle)screws with calcium apatite cement in patients with severe progressive osteoporotic spinal defomities: an innovative technique. European Spine Journal, 2000; 9 (6): 528-533.

Staal HM, Heyligers IC, Sluijs JA vd. Stem displacement during reduction of a dislocated cemented total hip arthroplasty with a polished tapered stem. Journal of Arthroplasty, 2000; 15 (7): 944-946.

Staal HM, Lieshout ON, Wuisman PIJM; Orthokwis, Nederlands Tijdschrift voor Orthopaedie, augustus 2000.

Staal HM, Bongers KJ. van antegraad naar retrograad, non-union van een supracondylaire femurfractuur bij een gebroken intramedullaire osteosynthese. Nederlands Tijdschrift voor Traumatologie, april 2003.

Wijnen W, Staal HM. Klompvoet behandeling in het azM. In dit verband, 2007; 17 (3): 14-17.

Staal HM, Gersen HJJ. Een moeilijke bevalling. In dit verband, 2007; 17 (1): 11. 


\section{Appendix}

Poelman TA, Staal HM, Willems WJ. A pseudo-iatrogenic case of medial clavicular fracture. Strategies Trauma Limb Reconstr, 2008; 3 (1): 23-25.

Vles GF, Vles JS, van Kleef M, van Zundert J, Staal HM, Weber WE, van Rhijn LW, Soudant D, Graham HK, de Louw AJ. Percutaneous radiofrequency lesions adjacent to the dorsal root ganglion alleviate spasticity and pain in children with cerebral palsy: pilot study in 17 patients. BMC Neurol, 2010 Jun 22; 10:52

Klaassens M, Reinstein E, Hilhorst-Hofstee Y, Schrander J, Malfait F, Staal H, Ten Have L, Blaauw J, Roggeveen H, Krakow D, De Paepe A, van Steensel M, Pals G, Graham J, Schrander-Stumpel C. Ehlers-Danlos arthrochalasia type (VIIA-B) expanding the phenotype: from prenatal life through adulthood. Clin Genet. 2011 Jul 29. doi: 10.1111/j.1399-0004.2011.01758.

Soudant DL, Staal HM, Witlox MA, Vles JS. Conscious sedation or general anaesthetic for intramuscular botulinum toxin injections in children, a two centre cross-sectional prospective audit. Eur J Paediatr Neurol. 2013 Mar;17(2):219-20. doi: 10.1016/j.ejpn.2012.06.012. Epub 2012 Aug 4.

Staal HM, Eertwegh vd V, Rhijn van LW. Communicatie train je op de werkvloer. Publicatie Medisch Contact Nr. 50 - 14 december 2012. Jaargang, 2012: 2855-2857

Prinzen L, Staal HM, Rouwette SJ, Beckers EA, Ten Broeke RH, van Rhijn LW, Henskens YM. Triple Red Blood Cell Alloantibody Formation After Bone-Allograft Transplantation. Am J Transplant. 2013 Jan;13(1):229-31.

Staal HM, van der Wijk J, Moh PAK, Rompa PJJM, van Rhijn LW. The M-osteotomie for Blount's disease, infantile tibia vara, a forgotten procedure? NTVO 2013; 20(4):106-109.

Van der Wijk J, Staal HM. Een Splinter. Rubriek Gezien, Medisch Contact april 2014. 
Van Rooij MJ, Staal HM, Klaassens M, van Rhijn LW, Stumpel CTRM. Essential timing of orthopaedic treatment in children with Ehlers-Danlos syndrome athrochalasia type: a case report, F1000 Research Articles, 6 april 2014.

Staal HM, Witlox MA, Mooij DT, Emans PJ, Ham SJ, van Rhijn LW, Welting TJM. Current Knowledge on Exostoses Formation in Hereditary Multiple Exostoses: Where do Exostoses Originate and in What Way is their Growth Regulated? Hereditary Genetics 2014, 3:3

Staal HM, Meijs N. Disease, bow legs of unknown origin and well-known treatment. MT 4, 2014.

Staal HM, Nijhuis W, Hannink G, Witlox MA, van der Zwan A, Ham SJ, Arts JJ, van Rhijn LW. Radiological follow up of distal femoral osteochondromas in patients with hereditary multiple osteochondromata (HMO). A longitudinal assessment of growth and location of osteochondromas. International Journal of Research Studies in Biosciences (IJRSB), vol 3 (8), aug 2015 PP 68-77

Staal HM, Goud AL, Van der Woude HJ, Witlox MA, Ham SJ, Dremmen MHG, Van Rhijn LW. Skeletal maturity of children with multiple osteochondromas: is diminished stature due to a systemic influence, Journal of Children's Orthopaedics: Volume 9, Issue 5 (2015), Page 397-402

Staal HM, Nijhuis W, Witlox MA, Boom K, Ham SJ, Welting TJM, Rietbergen B, Van Rhijn LW. The microarchitecture of bone in osteochondromas. A pilot study with micro computed tomography imaging on 9 osteochondromas. Journal of Bone Reports \& Recommendations 2015 Vol. 1 No. 2:10

Hollman F, Korpisah J, Ismail AH, Rompa PJJM, Moh P, Van Rhijn LW, Staal HM. W/M serrated osteotomy for infantile Blount's disease in Ghana: short term results' Nigerian Journal of Clinical Practice. 2015 
Appendix

Staal HM, Dremmen MHG, Robben SGF, Witlox MA, Van Rhijn LW. The use of Whole-Body MR Imaging in children with $\mathrm{HMO}$, an extended case study in two patients, Pediatric \& Therapeutics 2016, 6:1

\section{Book}

Bongers KJ, Staal HM, Kinderfracturen in beeld, Drukkerij van Genderen, Groningen, the Netherlands, ISBN 978-90-90226445-5, 2011. 
Appendix 


\section{DANKWOORD}

Laat ik dit dankwoord beginnen met de mensen dichtbij.

Lieve familie, een boek maken is leuk en ik besteed er veel werk er, maar samen zijn met jullie gaat boven alles.

Lieve Kasper, Pieter en Sarah, er is niets mooier dan met jullie samen te zijn, te lezen, te tekenen, te kaarten en samen de wereld te ontdekken. Samen ouder worden is een voorrecht.

Papa en mama, Wim Staal en Willemien Staal-Passchier, altijd ondersteunend en geïnteresseerd. Ik geniet van onze wekelijkse diners. Het leven wordt geleefd aan de goed gedekte tafel met familie en vrienden. Mien zorgzaam en Wim luisterend en sociaal. Een meer veilige thuisbasis had ik niet kunnen wensen. Jullie hebben me geleerd dat hard werken loont en zingevend is.

Wouter, altijd klaar om iedereen te helpen. Dank voor je immer snelle hulp bij het oplossen van alle logistieke problemen.

\section{Lieve vrienden}

Martine van der Weg, je kent me al mijn hele leven. Ik kan altijd op je rekenen, als een zorgzame oudere zus. Het huis is op orde, de kinderen onder de pannen en ook nog een bloemetje op tafel, zodat ik tijd heb om weg te zijn bij het gezin, te schrijven of weer eens ergens anders te zijn, Ghana, Helsinki of waar dan ook. En als ik dan moe thuis kom stuur je me op tijd naar bed. Merci.

Marie-Claire Hageman, heerlijk om samen bij te praten, na te denken, te evalueren en dat allemaal op of naast de mat. Je weet als geen ander zaken in perspectief te zetten. De afleiding geeft weer nieuwe energie en die kunnen we allebei goed gebruiken!

Monique Jacobs en Patrick van Battum, we zijn samen gestart in Nijmegen met onze academische carrière en we mochten samen genieten van de voorrechten van ons studentenleven. Monique je hebt gelijk, wij hebben het goed met alle kansen en luxe van ons leven. Het is een eer dat je mijn paranimf wil zijn. 


\section{Appendix}

Arnold Swinkels, super veel dank voor je hulp bij de laatste loodjes. De mooie strips die je hebt uitgewerkt en het stroomlijnen van alle losse eindjes. Dank je.

Anne-Marieke Staal en Jos Reinders, thank you guys for correcting the manuscripts! You are the best.

Lieve vrienden en familie om samen mee te spelen, te eten, te discussiëren, om moeilijke tijden mee te delen en om het leven mee te vieren; familie Muijs-Mayorga, Marianne van Heusden, familie Hageman-Linssen, de Room-Bergers, familie Postema-Weemhof, Carolien Frotscher en kinders Seegers, Anne-Marieke met Finn en Jos Staal-Reinders, Paul en Dominique Bude, familie Feczko-Stremersch, familie Staal, familie Passchier, familie Regter, familie Van der Weg, familie Castelein. Hopelijk kunnen we nog vele mooie momenten samen delen.

Lieve leraren, Wil Lüschen, Karel Gietelink, René Corten en Karst Bongers. Jullie zijn fantastische voorbeelden geweest voor mij en hebben ieder op jullie eigen manier mij kunnen inspireren. De eigenheid en eerlijkheid waarmee jullie je vak uitoefenen is belangrijk en mag een voorbeeld zijn voor velen. Wil, al die talloze trainingen en lessen en dan nog even een kampioenschap tussendoor, de boeken die we samen lazen en de vele gesprekken die we samen hebben gevoerd, altijd een filosofische blik op de weg die wij gaan en het waarom. Karel altijd in balans, de heerlijke afleiding met elkaar in de Ardennen of op het water, waar we het leven even laten voor wat het is en één worden met de natuur. Leraar en leerling, een verbondenheid die reikt over de grenzen van ruimte en tijd. Dank jullie voor alle lessen.

\section{Promotoren}

Prof. Dr. Van Rhijn, lieve Lodewijk, inspirator van dit onderzoek. Met veel enthousiasme en humor zijn we jaren geleden begonnen aan dit onderzoek. Jij kwam met het lumineuze idee om te kijken naar de groei van exostosen en naar de rol van het kraakbeen op de top. Samen zijn we ouder en wijzer geworden, al discussiërend over ontwikkeling, groei en rollen. Van cel tot mens en van een voorzichtig eerste idee, naar een compleet boekwerk. Je positieve energie en 
gedrevenheid zijn een stimulans zowel voor de wetenschap als voor de afdeling. Je kijk op persoonlijke en professionele groei maken je tot een inspirerend mens. Voor jou is een glas half vol of toch nog voller. Promotor, baas, collega en vriend, hopelijk kunnen we nog vele jaren samen bouwen aan een goede afdeling, regionaal orthopedisch onderwijs en aan groei binnen en buiten het ziekenhuis.

Dr. Welting, lieve Tim, jouw kennis over de cellen en eiwitten doet mij nog steeds duizelen. Je leerde mij meer begrip te hebben voor de cel en haar omgeving, om zo meer structuur te brengen in mijn creatieve gedachten. Door het onderzoek konden we de kliniek en het laboratorium samenbrengen. We leerden elkaar beter kennen in een roerige periode van onze levens. Op nieuwjaarsdag bij jou op de bank toonde jij jouw prachtige collectie kristallen, als telg van een echte mijnwerkersfamilie. Kristallen en eiwitten liggen mijlenver uit elkaar, net zoals de patiëntenzorg en de wetenschap, maar ze hebben allen een fascinerende glans. Hopelijk wordt het laboratorium groot in de kliniek en vice-versa. Laat het licht in goede gezondheid nog lang branden op de berg!

Dr. Witlox, lieve Adhiambo, wat een lange weg hebben we afgelegd. Ik agnio in de VU en jij "mijn" coassistent, allebei aios in dezelfde ROGO, jij al met een prachtige promotie op zak. Gelukkig wilde je de sprong naar het zuiden wagen, ook al is hier geen zee en geen open water, en zo werden we in het MUMC collega's. Je passie voor onderzoek is gebleven en als copromotor gaf je structuur aan mijn onsamenhangende ideeën. Dank voor je hulp bij het ordenen van de literatuur. Hopelijk kunnen we nog lange tijd samenwerken aan de ontwikkeling van de kinderorthopedie en staat er straks een prachtige onderzoekslijn naar epifysaire afwijkingen.

\section{Leescommissie}

Prof. Dr. Vles, lieve Hans, het is een eer dat je deze taak op je hebt willen nemen. Ik heb zoveel van je geleerd door je fantastische omgang met de kinderen in de kliniek. Je bent immer op zoek naar antwoorden, zowel binnen je vakgebied als daarbuiten. Dank. 


\section{Appendix}

Gewaardeerde overige leden van de leescommissie dank voor het beoordelen van het manuscript: Prof. Dr. Sjoerd Bulstra, Prof. Dr. Michiel de Haan, Dr. Jan van Mourik, Prof. Dr. Jeanine Verbunt. Hopelijk zullen onze wegen elkaar nog vaak kruisen in de kliniek, rondom de ziekte $\mathrm{HMO}$ en natuurlijk in het onderwijs.

\section{Medeauteurs}

Dr. John Ham, via jou kwam ik voor het eerst in aanraking met het ziektebeeld HMO. Een hele poli vol in het OLVG. Fantastisch dat je hebt willen meedenken en mede-beoordelen in de verschillende projecten die we hebben kunnen opzetten. Dank voor je materiaal en voor het delen van jouw jarenlange ervaring op het HMOterrein.

Arnoud van der Zwan, samen aios in het OLVG en nu samen aan het werk om een beter begrip te krijgen van de ziekte HMO. Dank voor de opbouwende gesprekken en ideeën over het onderzoek.

Prof. Dr. Simon Robben en Marjolein Dremmen, collega's van de kinderradiologie. Dank voor het beoordelen van alle röntgenfoto's en MRI's en voor het aandragen van de vele goede ideeën en bijzondere patiënten.

Wouter Nijhuis dank voor het reizen naar Eindhoven en het uitwerken van de röntgenstudies. Hopelijk kun je als fellow kinderorthopedie nog meer inzicht krijgen in de oorzaak van $\mathrm{HMO}$.

John Derwall, dank voor het inzetten van je kennis en innovatie om de losse plaatjes te maken tot video's en voor het aan elkaar knopen van de laatste eindjes.

Beste Dr. Pieter Emans, Dr. Ir. Bert van Rietbergen, Giovanni Paffen, Koen Boom, Drs. Annemarie Goud, Dr. Henk-Jan van der Woude, Dr. Gerjon Hannink, Tristan de Mooij, Dr. Chris Arts en Jasper Stevens, hartelijk dank voor jullie bijdrages aan de verschillende artikelen. 


\section{Onderzoekers en studenten}

Beste Reduan Elfedalli, Areej Alsadah, Brent van der Doelen en Kim van Someren, dank voor jullie hulp bij het verzamelen van de patiëntendata. Nina Wijnands dank voor je hulp bij het omzetten van de lijsten.

\section{Onderzoekers laboratorium en medewerkers van het wetenschappelijke trial bureau}

Don Surtel, Andi Cremers, Marjolein Caron, Liesbeth Jutten en Chris Arts, dank voor jullie hulp bij het verwerken van de data, het maken van foto's en voor de hulp bij het verwerken van de statistiek.

\section{Collega's}

Mark van den Boogaart, René ten Broeke, Pieter Emans, Peter Feczko, Jan Geurts, Joris Hermus, Inge van Rensen, Lodewijk van Rhijn, Paul Willems, Adhiambo Witlox, zeker niet op de laatste plaats dank aan jullie allen. We vormen samen een team en zonder jullie hulp was deze promotie niet tot stand gekomen. We werken hard om alle ballen in de lucht te houden: de patiëntenzorg, de wetenschap, het onderwijs en de nodige bestuurszaken. Een groep die bruist van de activiteit. ledereen met zijn eigen thema en met zijn eigen aandachtsgebied, als raderen in het netwerk, of zoals het motto van Alexander Dumas' Drie Musketiers: "een voor allen, allen voor een".

Wijlen Jan-Willem Duyzings, kamergenoot, ik vind het heel jammer dat ik je dit werk niet meer kan laten zien. Je toonde mij de kracht die je bezat om met plezier en humor te werken, ook bij tegenslagen. Ik mis de memo-briefjes op mijn bureau.

\section{Secretariaat en planning}

Beste dames van het secretariaat en planning Marion Bastings-van Wissen, Wil van Erk-Janssen, Manon Degroof-Luijten, Chantalle Gadet-Schoenmakers, Jerney Willems, Marieke Prins en Caren Cheriex, jullie zijn de spil van onze organisatie. Post, telefoon, brieven, afspraak, cursus of congres en dan ook nog even dit en even dat tussendoor. Nooit te beroerd om nog iets voor me op te zoeken of uit te printen. Wil, dank voor je hulp bij het photo-shoppen van de afbeeldingen. Dank jullie allen voor de planning en organisatie. 


\title{
Appendix
}

\section{Poli en ondersteuning}

Alle medewerkers van de poli orthopedie in het bijzonder Armand Beckers en de ondersteuners Helma Voets, Wiel Wijnen en Mieke Vandewall-Peeters, dank voor het organiseren van de spreekuren en het te woord staan van de patiënten en natuurlijk vooral voor het plannen en organiseren van alle extra poli's en de onderzoeken voor de HMO/HME poli.

\begin{abstract}
Aios
Jullie vragen om uitleg zorgen ervoor dat we altijd terug gaan naar de basis van ons vak. Hoe leergieriger jullie zijn, hoe groter onze gezamenlijke kennis wordt. Het is inspirerend om samen te leren.
\end{abstract}

Dank aan alle anderen die een bijdrage aan dit werk hebben geleverd en die ik vergeten ben te benoemen. 


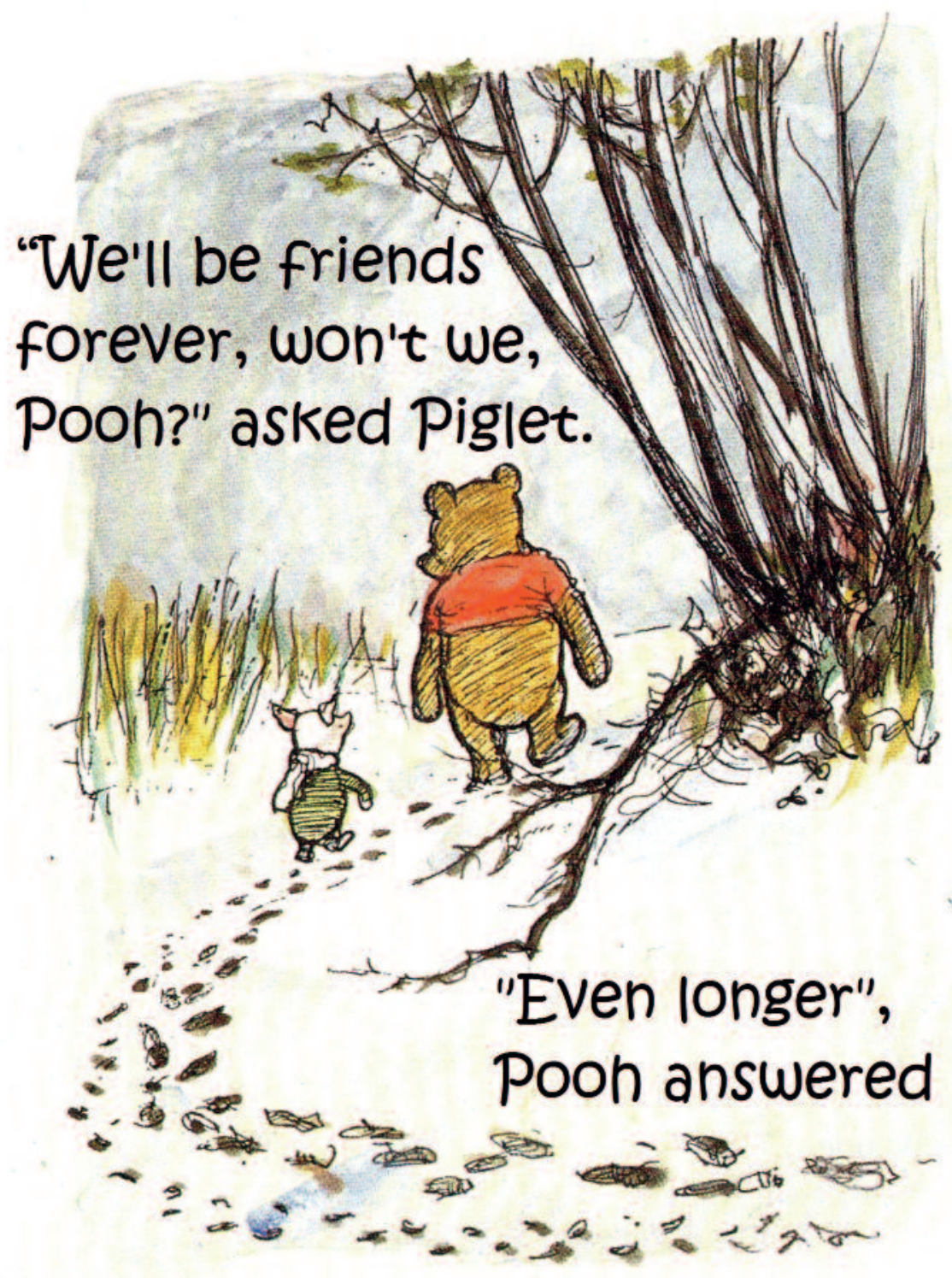


